\title{
Manipulating niche composition limits damage to haematopoietic stem cells during Plasmodium infection
}

\author{
Myriam L. R. Haltalli ${ }^{1,2,3,4}$, Samuel Watcham ${ }^{\circledR 3,4}$, Nicola K. Wilson ${ }^{3,4}$, Kira Eilers ${ }^{1}$, Alexander Lipien', \\ Heather Ang ${ }^{1}$, Flora Birch ${ }^{1,2}$, Sara Gonzalez Anton ${ }^{1,2}$, Chiara Pirillo, ${ }^{1,2}$, Nicola Ruivo ${ }^{1}$, Maria L. Vainieri ${ }^{1,7}$, \\ Constandina Pospori, ${ }^{1,2}$, Robert E. Sinden', Tiago C. Luis ${ }^{1}{ }^{1}$, Jean Langhorne ${ }^{2}$, Ken R. Duffy ${ }^{5}$, \\ Berthold Göttgens $\mathbb{D}^{3,4}$, Andrew M. Blagborough ${ }^{6}$ and Cristina Lo Celso ${ }^{1,2}{ }^{\square}$
}

\begin{abstract}
Severe infections are a major stress on haematopoiesis, where the consequences for haematopoietic stem cells (HSCs) have only recently started to emerge. HSC function critically depends on the integrity of complex bone marrow (BM) niches; however, what role the BM microenvironment plays in mediating the effects of infection on HSCs remains an open question. Here, using a murine model of malaria and combining single-cell RNA sequencing, mathematical modelling, transplantation assays and intravital microscopy, we show that haematopoiesis is reprogrammed upon infection, whereby the HSC compartment turns over substantially faster than at steady-state and HSC function is drastically affected. Interferon is found to affect both haematopoietic and mesenchymal BM cells and we specifically identify a dramatic loss of osteoblasts and alterations in endothelial cell function. Osteo-active parathyroid hormone treatment abolishes infection-triggered HSC proliferation and-coupled with reactive oxygen species quenching-enables partial rescuing of HSC function.
\end{abstract}

$\mid$ aematopoietic stem cells (HSCs) reside within the bone marrow (BM) where they interact with multiple stroma and haematopoietic cell types. These cells form complex niches that regulate HSC function by maintaining quiescence and supporting their self-renewal ${ }^{1}$. Once outside the niche, HSC progeny differentiate into highly proliferative progenitor cells, which further develop into erythroid, megakaryocyte, myeloid and lymphoid lineages.

Infection is a natural stressor of the haematopoietic system, whereby haematopoietic stem and progenitor cells (HSPCs) modify their progeny output to promptly cope with the increased demand for mature cells $s^{2-5}$. Infectious challenges result in hallmark HSPC responses, including expansion of the early progenitor compartment (Lineage ${ }^{-}{ }^{-}-\mathrm{Kit}^{+} \mathrm{Sca}-1^{+}$; also known as LKS cells) and alterations in HSC cycling properties, long-term function and migration patterns $s^{6-9}$. It is widely accepted that increased proliferation leads to loss of HSC function, but it is unclear whether during infections other factors may contribute to HSC decline. These phenomena happen within the BM microenvironment, yet little is known about the role of HSC niche components in regulating the observed changes in HSPC dynamics. With severe infections on the rise worldwide, it is important to devise therapeutic strategies that preserve HSC function, including through targeting the niche.

Malaria is a life-threatening disease, with an estimated 216 million new cases in 2016 resulting in approximately 445,000 deaths ${ }^{10}$. Plasmodium infects and destroys red blood cells and has notable impacts on erythropoiesis ${ }^{11-14}$, and the haematopoietic/ immune function of malaria survivors is compromised both short and long term ${ }^{15,16}$ due to alterations in myeloid lineage cells ${ }^{15,17}$. It is likely that these effects originate from changes at the earliest stages of haematopoiesis, including the HSC compartment. So far, however, little work directly addresses the effect of Plasmodium infection on primitive HSPCs. Using the murine experimental model Plasmodium berghei, we previously demonstrated that multiple components of the haematopoietic tree simultaneously respond with a substantial increase in proliferation during advanced blood stage infection' This raised questions about what cell-intrinsic or -extrinsic mechanisms mediate the observed haematopoietic dynamics, how this affects HSC long-term function and whether infection-induced changes to the BM microenvironment contribute to the phenotypes observed.

Here, we combine molecular, phenotypic, functional and intravital microscopy (IVM) analyses to study the haematopoietic and BM microenvironment responses over the course of infection, investigate causal links between the two and test whether niche manipulation is a viable approach to rescue HSC function.

\section{Results}

P. berghei infection leads to loss of HSC transcriptional identity and function. Mice infected by bites of mosquitos carrying $P$. berghei sporozoites are an excellent experimental model to study the effect of severe malaria on haematopoiesis?. Despite variability in parasitaemia, flow cytometry analysis of BM from control and infected mice highlighted a dramatic increase in the number of LKS cells by day 7 post-sporozoite infection (psi) (Fig. 1a).

To obtain a broad and unbiased understanding of the cellular and molecular changes taking place in HSPCs as a consequence 
of $P$. berghei infection, we performed single-cell RNA sequencing (scRNA-seq) on Lineage ${ }^{-} \mathrm{c}-\mathrm{Kit}^{+}$cells from control mice and mice at day 7 psi (Fig. 1b). Similar numbers of cells were analysed from two control and two infected animals with average parasitaemia for the disease stage (Fig. 1a). Over 7,000 cells passed quality control in all samples, and a median of over 2,400 genes were detected per cell (Fig. 1b). We grouped control cells into clusters (Extended Data Fig. 1a-f) and mapped cells from infected animals against their corresponding control cluster. Force-directed graphs ${ }^{18}$ highlighting the various lineage branches (Fig. 1c) indicated that, in infected mice, early haematopoiesis was substantially rewired towards myeloid and basophil lineages, at the expense of primitive cells and erythroid and megakaryocyte lineages (Fig. 1d and Extended Data Fig. 1g). These data can be further explored on our interactive website. Next, we determined that machine learning-based HSC scores $^{19}$ were strikingly decreased for cells from infected mice (Fig. 1e and Extended Data Fig. 1h), raising questions about how P. berghei affects HSC function.

To perform functional analyses on relatively homogeneous cell populations whose properties are well understood at steady-state, we focused on the LKS CD $150^{+} \mathrm{CD} 48^{\text {low/neg }}$ phenotypic cell population, which is enriched for HSCs but includes primitive multipotent progenitors (MPPs) ${ }^{20-23}$. Here, we label cells with this phenotype signalling lymphocyte activation molecule (SLAM) HSPCs and collectively label the remaining cells within the LKS population MPPs (Fig. If and Extended Data Fig. 2a,b). Transplantation of purified SLAM HSPCs from control and infected CD45.1 donor mice, together with limited numbers of CD45.2 whole BM (WBM) support cells, into lethally irradiated CD45.2 recipients (Fig. 1g) indicated that cells from infected animals had drastically reduced engraftment ability (Fig. 1h); however, their long-term multilineage potential was largely consistent with that of transplanted control SLAM HSPCs (Fig. 1i). Together with the scRNA-seq, these data show an extensive loss of HSC transcriptional identity and functionality upon infection, raising questions about what mechanisms trigger this extensive damage.

$P$. berghei infection affects the population dynamics of early HSPCs. To gain a quantitative understanding of HSPC dynamics as infection progresses, we employed an established dual-pulse labelling method $^{23}$. Consistent with previous observations ${ }^{9}$, the Lineage ${ }^{-} \mathrm{c}-\mathrm{Kit}^{+} \mathrm{Sca}-1^{-}$compartment had an increased proliferation rate but a reduced size by day 7 psi, whereas proliferation of both MPPs and SLAM HSPCs was markedly increased by day 5 (Extended Data Fig. 2c-e; circles in Fig. 2a). Unlike MPPs, SLAM HSPC numbers remained constant throughout the infection (Extended Data Fig. 2e; circles in Fig. 2b), despite increased proliferation.

To test the contribution of proliferation and differentiation to each compartment, we performed quantitative inference via a simple mathematical model based on the collected data (Fig. 2a,b and Supplementary Note). This demonstrated that SLAM HSPC contribution alone is not sufficient to account for the growth of the MPP population observed experimentally. This instead is consistent with MPP progeny remaining within the same compartment up to day 5 , after which differentiation accelerates. Next, we assessed the effect of proliferation on the turnover of SLAM HSPC and MPP cell compartments, assuming that all cells within a compartment cycled sequentially. In control mice, the maximum number of SLAM HSPCs undergoing the Sphase at least once within $8 \mathrm{~d}$ constituted less than the whole compartment (Fig. 2c). However, in infected mice, by day $5 \mathrm{psi}$, the whole SLAM HSPC compartment could have already turned over once, and by day $8 \mathrm{psi}$, it is possible that the whole SLAM HSPC population will have turned over approximately five times as a result of the faster proliferation rates. The MPP compartment could turnover once every $8 \mathrm{~d}$ in control mice, but this would increase up to 40 times during infection (Fig. 2c). These data show that $P$. berghei infection drastically increases HSPC turnover and, together with the transcriptomic analysis and transplantation assays, suggest a mechanism for the loss of functional SLAM HSPCs in response to severe infection.

Next, we deepened our flow cytometry analyses to focus on immunophenotypes highly enriched for the most functional HSCs; namely, endothelial protein C receptor-positive (E-SLAM) $\mathrm{HSCs}^{24}$ (Extended Data Fig. 2f) and LKS CD $150^{+} \mathrm{CD} 48^{\text {neg }} \mathrm{HSCs}\left(\mathrm{CD} 48^{\text {neg }}\right.$ HSCs $^{23}$ (Extended Data Fig. 2g). The size of each population decreased in infected mice (Fig. $2 \mathrm{~d}, \mathrm{e}$ ) and the proliferation rate of the surviving CD48 neg $\mathrm{HSCs}$ increased substantially (Fig. 2f). However, our mathematical analysis indicated that, by day $8 \mathrm{psi}$, $85 \%$ of the $\mathrm{CD} 48^{\text {neg }}$ HSC compartment would have been replaced by completely new cells, compared with $30 \%$ in the control (Fig. $2 \mathrm{~g}$ ). These data highlighted that, although $P$. berghei induces the proliferation of more quiescent HSC subsets, it does not cause the entire compartment to turnover. Proliferation alone is therefore unlikely to cause the near-complete loss of HSC function observed.

\section{P. berghei-exposed HSPCs and BM stromal cells exhibit a strong} interferon (IFN) response. To identify molecular mechanisms driving HSC damage, we re-examined the scRNA-seq dataset to determine differentially expressed genes (DEGs) between control and infected samples. Filtering for genes at least fourfold upregulated in all clusters identified 20 initial driving genes, and the list grew to 109 when including DEGs whose expression correlated highly (Supplementary Table 1). SPRING analysis highlighted the role of these genes in separating HSPCs from healthy and infected animals (Fig. 3a). Most of the genes were related to IFN, and some specifically to IFN- $\gamma$ signalling (Fig. 3b,c and Extended Data Fig. 3a). Interestingly, when comparing DEGs in the overall HSPC population or in specific clusters, it became apparent that the driving genes were indeed upregulated in all clusters (Extended Data Fig. 3b,c). Downregulated genes were more numerous and less shared between clusters, consistent with haematopoietic rewiring requiring the

Fig. 1| P. berghei infection alters HSC transcriptional identity and function. a, Parasitaemia in peripheral blood over the course of infection versus the absolute number of LKS cells. Each circle represents one mouse and stars indicate mice analysed by scRNA-seq (control: $n=10$; day 3: $n=20$; day 5: $n=27$; day 7: $n=42$ ). The data were pooled from more than three independent infection experiments. $\mathbf{b}$, Gating strategy used to isolate Lineage ${ }^{-} c-K_{i t}{ }^{+}$ cells for droplet-based scRNA-seq at day $7 \mathrm{psi}$. The boxes indicate the number of cells that passed quality control and the median number of genes detected per cell per sample. c, Cluster identity of cells from control and infected mice. Using Louvain clustering on the k-nearest-neighbour graph, control cells were organized into six populations, as specified in the legend. Infected cells were mapped to their corresponding control cluster. The proportions of each cluster in both conditions are shown. $\mathbf{d}, \log _{2}$ [fold change] (positive values: increase; negative values: decrease) of the percentage of infected cells mapped to each cluster divided by the percentage of control cells in the same cluster. Left- and right-hand bars indicate fold changes for samples from two separate infected mice. MEP, megakaryocyte-erythroid progenitor. e, HSC score values plotted on the force-directed graph embedding for all cells from control and infected mice. f, Representative flow cytometry plots showing the gating strategy for MPP and SLAM HSPC populations in control and infected mice at day 7 psi. $\mathbf{g}$, Schematic of the transplantation assay presented in $\mathbf{h}$. h,i, Overall peripheral blood reconstitution (h) and multilineage output (i) of transplanted SLAM HSPCs from control and infected donors into lethally irradiated recipients, as assessed by flow cytometry. The data were pooled from recipients of two independent donor groups from two infections $\left(n=12\right.$ recipients per group). The data are presented as means \pm s.e.m. ${ }^{\star} P<0.05$; ${ }^{\star \star \star \star} P<0.0001$. The $P$ values were determined by two-way ANOVA with post-hoc Bonferroni correction (h) or unpaired two-tailed Student's $t$-test (i). 
inhibition of programs linked to several lineages and the initiation of a common transcriptional program. Gene Ontology analysis of primitive HSPC cluster DEGs further highlighted the prominence of IFN- $\gamma$-mediated signalling pathways, together with cell-cycle regulators, type I IFN and tumour necrosis factor (TNF) signalling, and metabolism-related genes (Fig. 3d and Supplementary Table 2).

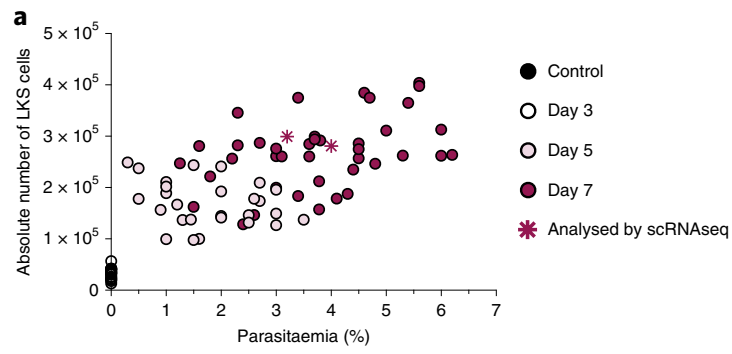

b
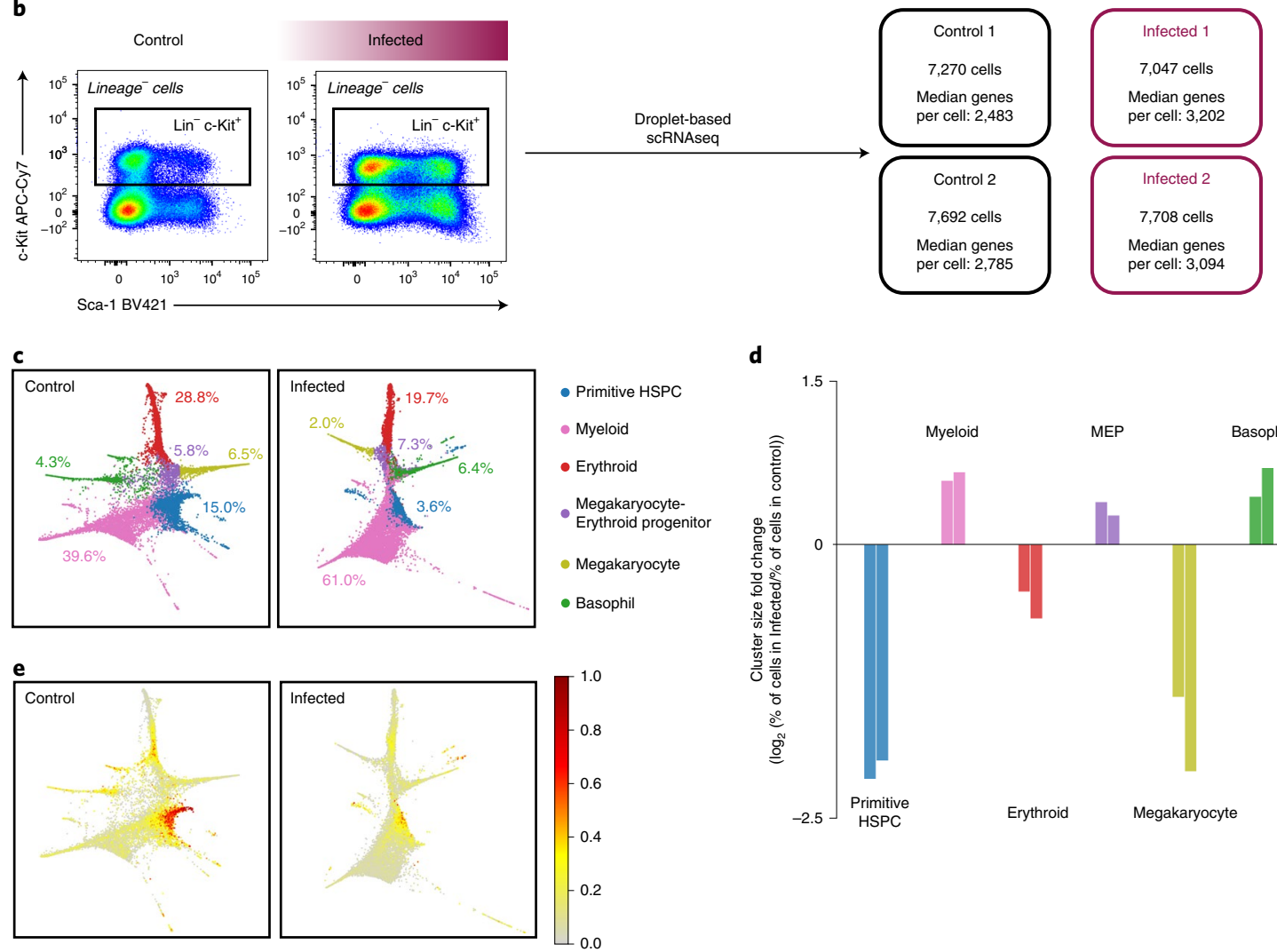

d

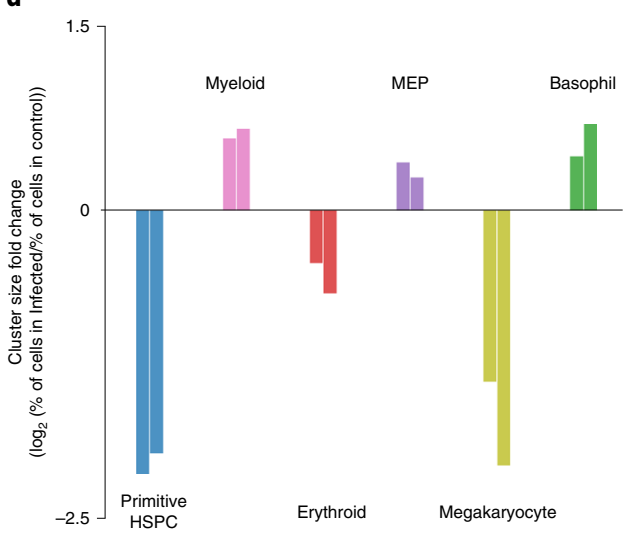

f

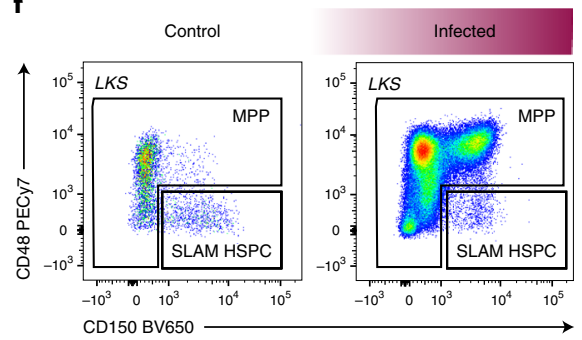

g
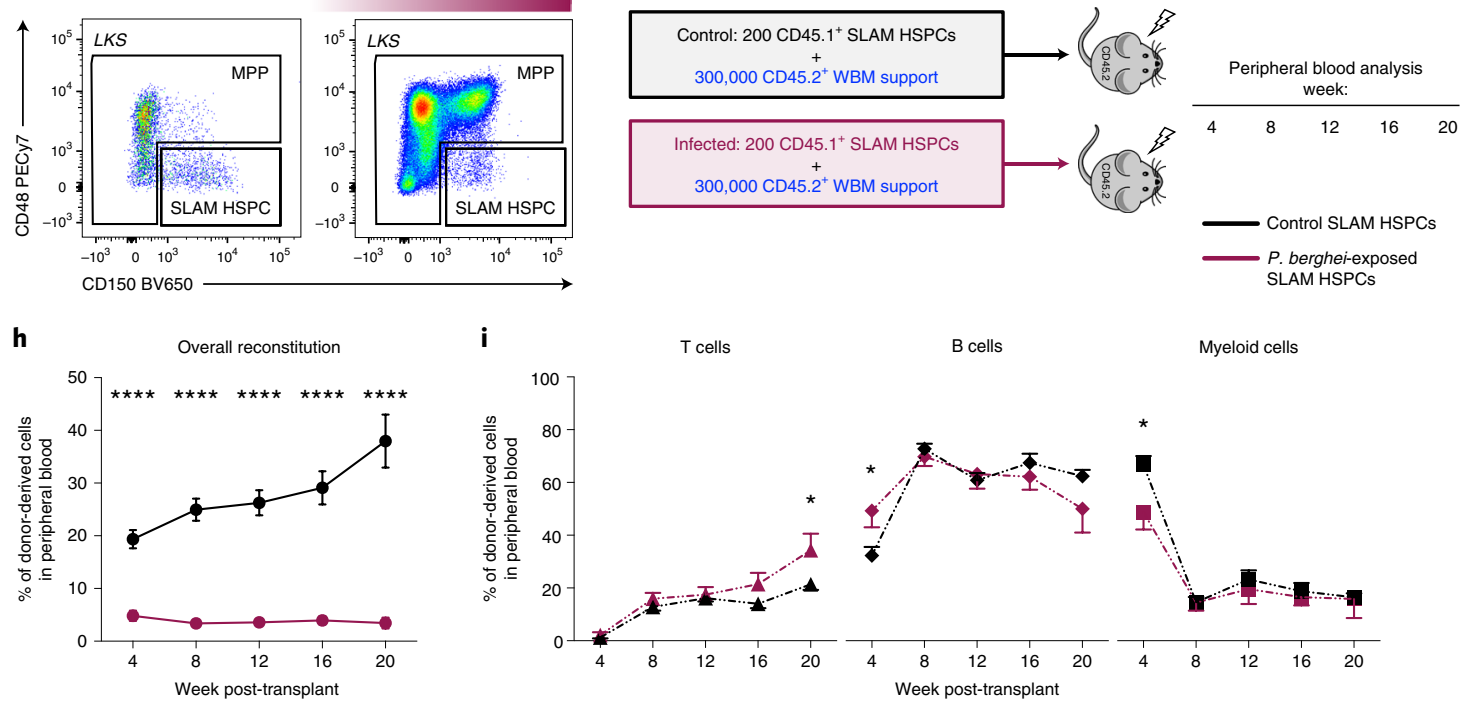

B cells

Myeloid cells 

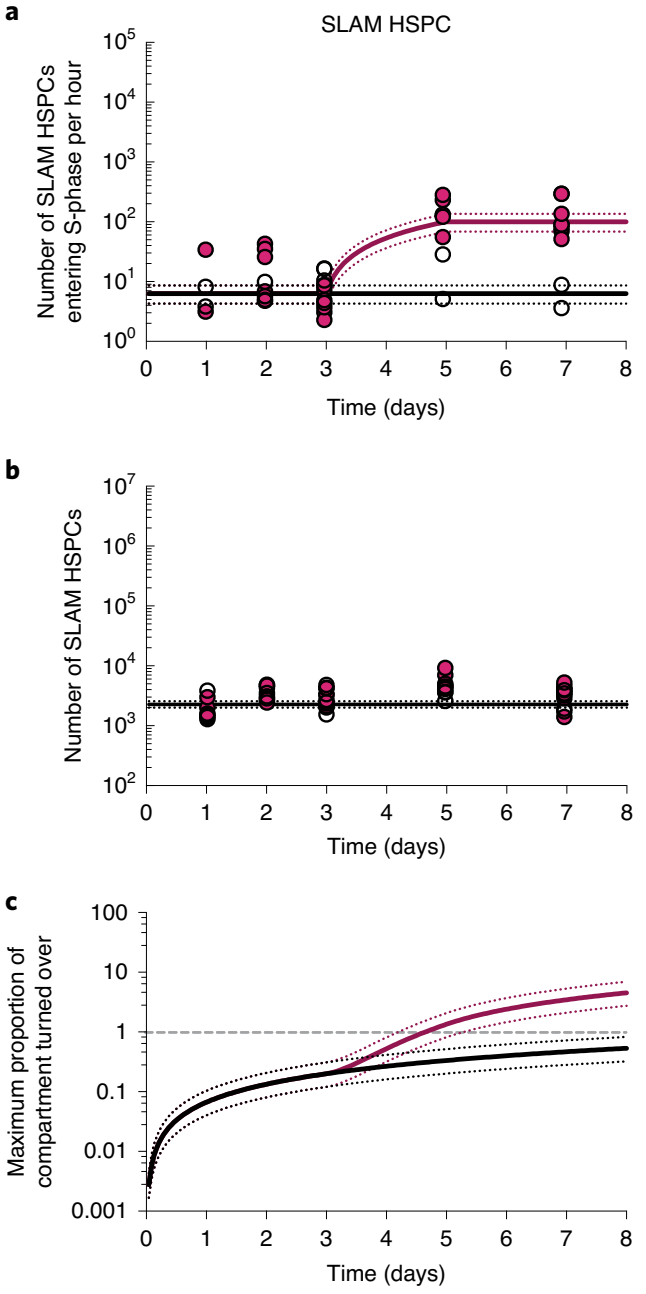
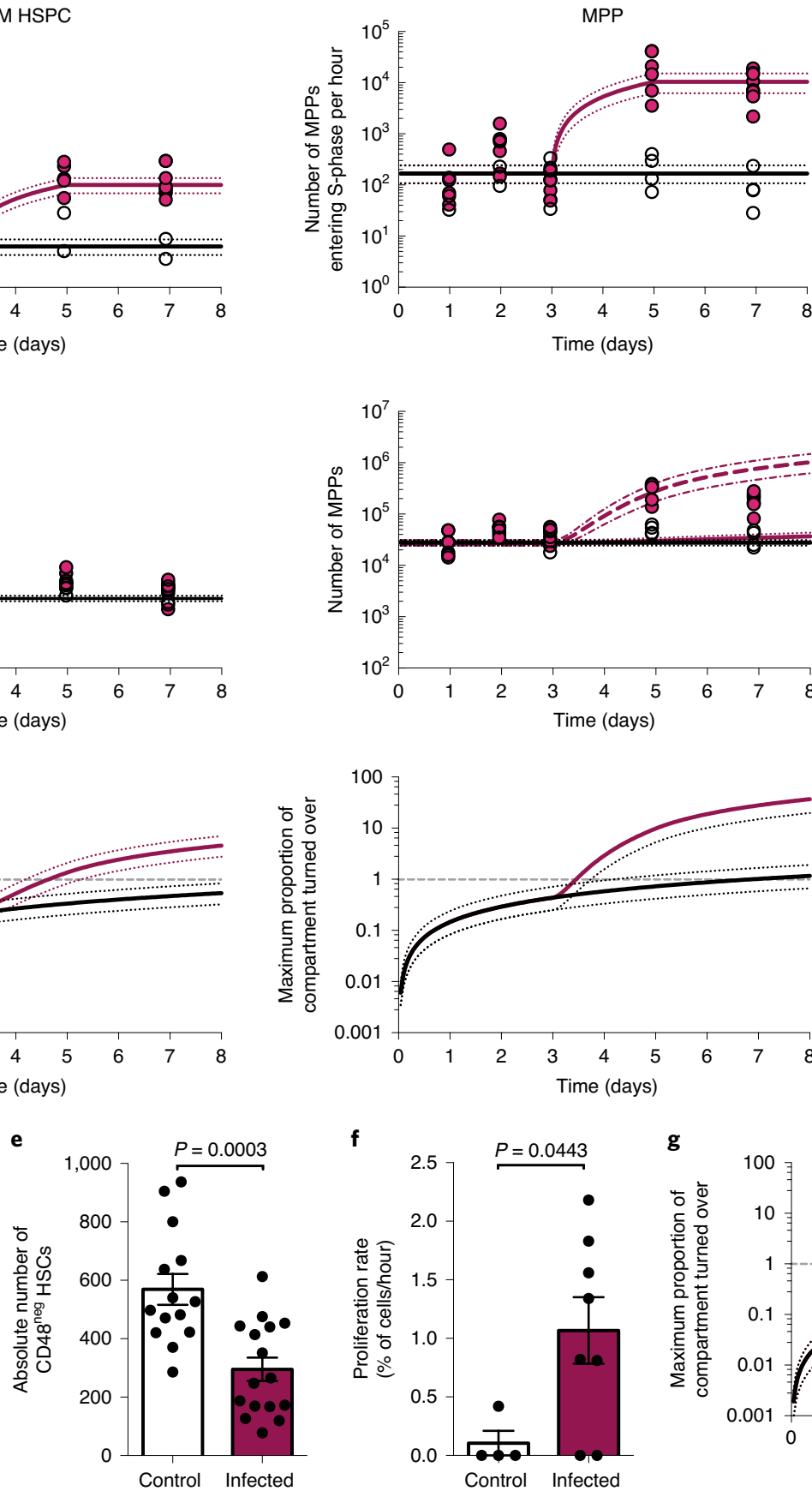

- Control model

- Infected model

O Control experimental data

O Infected experimental data

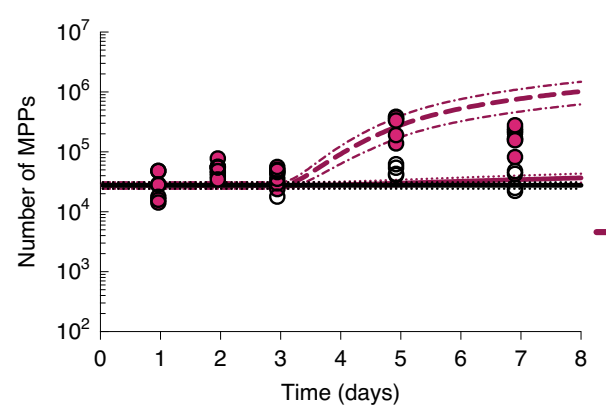

- Control model

- Infected model SLAM HSPC progeny is MPP and MPP progeny move out of MPP compartment

Infected model Both SLAM HSPC and MPP progeny are MPP
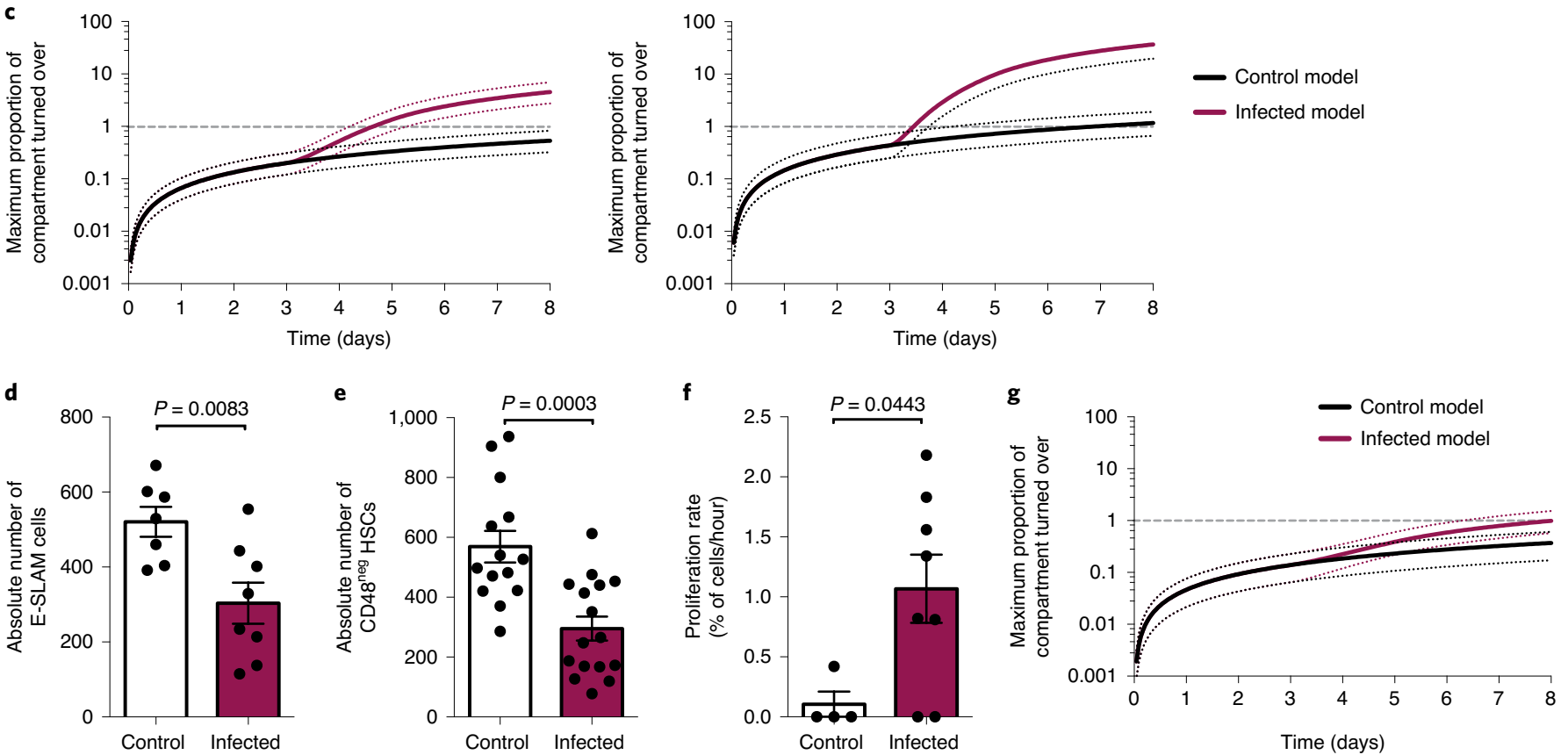

Fig. 2 | P. berghei affects population dynamics of early HSPCs. a, Experimental data and mathematical model representing SLAM HSPC and MPP proliferation rates. Proliferation is constant in the control model, corresponding to the average of measurements from all mice until day 3 psi. In the infected model, proliferation is constant until day 3 , then linearly increases to the average of the data from infected mice at days 5 and 7 psi. b. Experimental data and mathematical model of SLAM HSPC and MPP compartment sizes. SLAM HSPCs are modelled as a constant corresponding to the average data from all animals. For MPPs, the control model is a constant corresponding to the average of measurements from all control plus infected mice up to day 3 psi. In the first infected model (continuous maroon line), the number is constant with the control model until day 3 , then increases corresponding to the additional SLAM HSPCs entering the S phase in the infected model, as shown in a. In the second infected model (dashed maroon line), the number is constant until day 3, then increases based on the sum of additional SLAM HSPCs and MPPs entering the $S$ phase in the infected model, as shown in a. c, Modelling of the maximum proportion of SLAM HSPCs and MPPs turned over as a function of time in control and infected mice. Note that a log scale has been used for the $y$ axis. Intersections with the grey dashed line visually represent when the entire compartment could have completed one turnover. d,e, Absolute numbers of E-SLAM cells ( $n=7$ controls; $n=8$ infected mice) (d) and CD48 neg HSCs ( $n=14$ controls; $n=16$ infected mice) (e) at day 7 psi. f, Proliferation rate of CD $48^{\text {neg }} \mathrm{HSCs}$ at day $7 \mathrm{psi}(n=4$ control; $n=8$ infected mice). In d-f the data are presented as means \pm s.e.m. and the Pvalues were determined by unpaired two-tailed Student's $t$-test. $\mathbf{g}$, Modelling of the maximum proportion of CD48 ${ }^{\text {neg }}$ HSCs turned over as a function of time in control and infected mice. These data were pooled from up to three independent infection experiments. In $\mathbf{a}-\mathbf{c}$ and $\mathbf{g}$, the dotted lines indicate 95th percentile bootstrap confidence intervals for each model. 
To validate the role of cytokines, we measured IFN- $\gamma, \operatorname{IFN}-\alpha$ and TNF- $\alpha$ levels. We detected a striking increase of IFN- $\gamma$ in the serum and the BM supernatant of infected animals (Fig. 3e). IFN- $\alpha$ and TNF- $\alpha$ levels were also augmented (Extended Data Fig. $3 d, e)$, albeit to a lesser extent than IFN- $\gamma$. This was consistent with IFN- $\gamma$ being most widely associated with responses to Plasmodium infection in mice and humans ${ }^{25-28}$.

Based on this and given previous reports highlighting the complex effects of IFN- $\gamma$ on HSCs and niche components ${ }^{29}$, we investigated how these effects may be coordinated, and whether the niche may be a key mediator. We tested whether not only HSPCs, but rather all BM cells-including stroma-would sense and respond to the elevated systemic and local IFN- $\gamma$ in our model by measuring Sca-1 upregulation as a proxy of IFN- $\gamma$ signalling ${ }^{30}$. Haematopoietic cells $\left(\mathrm{CD} 45^{+}\right.$and/or Ter $\left.119^{+}\right)$were reduced in number and, as expected, had substantially upregulated Sca-1 (Fig. $3 \mathrm{f}-\mathrm{h}$ ). While the overall number of CD45-Ter119- mixed haematopoietic and stromal cells ${ }^{31}$ was not affected (Fig. 3i), the majority of these cells also upregulated Sca-1 (Fig. 3j,k).

To dissect the importance of direct IFN- $\boldsymbol{\gamma}$ action on HSPCs versus an indirect action mediated by changes in the BM microenvironment, we studied IFN- $\gamma$ receptor 1 (Ifngr 1) knockout and chimeric mice. Consistent with previous studies, we observed no difference in SLAM HSPC and MPP compartment size and proliferation rate in control and infected Ifngr $1^{-/-}$mice (Extended Data Fig. $4 a, b)$ despite the presence of high levels of IFN- $\gamma$ (Extended Data Fig. 4c). Next, we generated reverse chimeras and two groups of 50:50 mixed BM chimeras (Fig. 31). Upon infection, IFN- $\gamma$ levels were notably higher in all three chimera types (Extended Data Fig. $4 \mathrm{~d}-\mathrm{f}$ ). In the reverse chimeras, the number of total SLAM HSPCs was unchanged (Fig. $3 \mathrm{~m}$, left panel) and their proliferation rate increased upon infection (Fig. $3 \mathrm{n}$, left panel). In the mixed chimera groups, we analysed Ifngrl-deficient SLAM HSPCs. Their absolute numbers were unchanged in both groups (Fig. $3 \mathrm{~m}$, middle and right panels). SLAM HSPCs became highly proliferative upon infection in the presence of an Ifngr1-proficient niche (Fig. 3n, middle panel). Interestingly, this response was suppressed in mixed chimeras with an Ifngrl-deficient niche (Fig. $3 \mathrm{n}$, right panel). These data highlight that indirect, niche-mediated mechanisms contribute to HSC proliferation during infection, prompting further investigation into morphological and/or functional changes in the BM microenvironment.

Targeting the osteolineage inhibits HSC proliferation in response to $P$. berghei infection. Osteoblasts have long been associated with HSC maintenance ${ }^{32-35}$. IVM of the calvarium BM of control and infected Col2.3-GFP osteoblast reporter mice ${ }^{36}$ revealed a progressive and systemic loss of green fluorescent protein-positive $\left(\mathrm{GFP}^{+}\right)$ cells throughout infection (Fig. 4a,b). To confirm that this reflected loss of osteoblasts, we examined histological sections of femurs. While endosteum-lining cells were visible in control sections, they were undetectable in infected ones (Extended Data Fig. 5a,b).

We reasoned that, by targeting the osteolineage, one may control HSC proliferation and rescue HSC function during infection. Intermittent treatment with parathyroid hormone (PTH) enhances bone formation and increases osteoblast numbers by stimulating their proliferation, inhibiting apoptosis and driving osteoblast progenitor differentiation ${ }^{37-41}$. We treated mice with PTH for 2 weeks before and a further week during infection, before sacrifice and analysis (Fig. 4c). While PTH had no effect on parasitaemia (Extended Data Fig. 5c), it had an anabolic effect on the osteolineage, and a substantial number of osteoblasts survived through infection, as measured by IVM tilescans and flow cytometry analyses of long bones (Fig. $4 \mathrm{~d}-\mathrm{f}$ ).

To investigate the effects of PTH treatment on HSPC population dynamics, we measured their size and proliferation rate. Consistent with Sca-1 upregulation occurring in both groups of infected mice (Extended Data Fig. 5d), the absolute numbers and proliferation rates of Lineage ${ }^{-} \mathrm{c}-\mathrm{Kit}^{+} \mathrm{Sca}-1^{+}$cells (Extended Data Fig. 5e,f) and MPPs (Fig. 4g,h, left panels) were similar in all infected animals. However, PTH treatment had a specific effect on SLAM HSPCs. In line with previous studies ${ }^{32,42,43}$, in control mice, PTH treatment alone increased the number of SLAM HSPCs without affecting their proliferation rate (Fig. $4 \mathrm{~g}$, h, right panels). Importantly, SLAM HSPCs from PTH-treated infected mice proliferated as little as those of control animals and strikingly less than those of phosphate-buffered saline (PBS)-treated infected mice.

To test whether PTH treatment could rescue HSC function, we performed transplantation assays with SLAM HSPCs purified from PBS- and PTH-treated infected donors at day 7 psi. Surprisingly, we observed no difference between the engraftment and multilineage output of the two populations, and the overall donor-derived reconstitution was very low (Fig. 4i and Extended Data Fig. 5g). When we measured the numbers of CD48 ${ }^{\text {neg }}$ HSCs in PTH-treated mice, we observed that their abundance was markedly reduced in infected animals (Fig. 4j), despite proliferation not being induced. Interestingly, systemic and local IFN- $\gamma$ levels were higher in PTH-treated infected mice compared with PTH-treated non-infected mice, but were lower than those of PBS-treated infected animals (Fig. 4k,1). Because PTH has immunomodulatory properties and T cells have been described to contribute to the anabolic processes triggered by this hormone ${ }^{42,44}$, we analysed $\mathrm{T}$ cells in the BM and blood of PBS- and PTH-treated mice. While there were no differences in the absolute number of T cells and there was no change in the proportion of T cell subsets in the BM of PTH-treated mice (Fig. $4 \mathrm{~m}, \mathrm{n}$ ), there was a striking decrease in the proportion of activated $\mathrm{CD}^{+}$T cells in PTH-treated infected animals compared with those that were PBS treated (Fig. 4o). Further analyses revealed

Fig. $3 \mid$ P. berghei-exposed HSPCs and BM stromal cells exhibit a strong IFN response. a, Single-force graph embedding of combined control and infected cells, highlighting the large separation between samples according to their infection status, regardless of cell type. Recalculating the force graph with the driving genes removed suggests that they play a major role in driving this parting. b,c, Violin plots showing the expression of Irf7 (b) and Gbp2 (c), as determined by scRNA-seq analysis in control (black; left panels) and infected samples (maroon; right panels) ( $n=14,193$ control cells; 13,905 infected cells). The central dots show median values and the box limits show the upper and lower quartiles. The whiskers extend from the box limit to the smallest and largest value at no further than $1.5 \times$ the interquartile range from the hinge. d, Representative results from a Gene Ontology analysis of biological processes enriched in samples sequenced from infected mice compared with controls. $P_{\text {adj }}$ adjusted $P$ value. e, IFN- $\gamma$ levels measured by ELISA in serum and BM supernatant at day $7 \mathrm{psi}(n=3$ mice per group). Ctrl, control; inf, infected. f, Absolute number of CD45+Ter119+ haematopoietic cells at day 7 psi ( $n=9$ controls; $n=12$ infected mice). g,h, Representative histogram plot (g) and absolute numbers (h) of CD $45^{+}$Ter119+5ca- $1^{+}$cells at day 7 psi $(n=9$ controls; $n=12$ infected mice). $\mathbf{i}$, Absolute numbers of CD45-Ter119- mixed haematopoietic and stromal cells at day 7 psi $(n=9$ controls; $n=12$ infected mice). j,k, Representative histogram plot (j) and absolute numbers (k) of CD45-Ter119-Sca- $1^{+}$cells at day 7 psi $(n=9$ controls; $n=12$ infected mice). I, Schematic detailing the generation of Ifngr ${ }^{-1-}$ reverse and mixed chimera groups that were subsequently infected and analysed. $\mathrm{KO}$, knockout; $\mathrm{WT}$, wild type. $\mathbf{m}, \mathbf{n}$, Absolute numbers $(\mathbf{m})$ and proliferation rates $(\mathbf{n})$ of wild-type $\left(n=9\right.$ controls; $n=7$ infected mice) versus Ifngr $7^{-1-}(n=5$ controls; $n=5$ infected mice) SLAM HSPCs from chimeric mice. These data were pooled from up to three independent infection experiments. All of the data are presented as means \pm s.e.m. The $P$ values were determined by unpaired two-tailed Student's $t$-test. NS, not significant. 
overall lower proportions of both $\mathrm{CD}^{+}$and $\mathrm{CD} 8^{+} \mathrm{T}$ cells producing IFN- $\gamma$ in PTH-treated infected mice compared with PBS-treated mice (Fig. 4p). This was reflected by Tcells analysed in the blood of these mice (Extended Data Fig. 5h-k). We observed this effect on T cells only following the prolonged, anabolic PTH treatment regimen, and not when $\mathrm{PTH}$ was administered exclusively for the week following infection initiation (Extended Data Fig. 6). This raises the interesting hypothesis that, in this context, PTH may not act directly or acutely on T cells, but rather its immunomodulatory effects may be niche mediated too. Together, these data suggest that, although prolonged PTH treatment leads to partial retention of osteoblasts, avoidance of infection-induced HSC proliferation and a

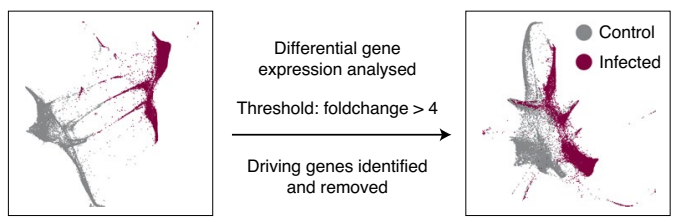

d Cytokine-mediated signaling pathway (GO:0019221)

Cellular response to type I interferon (GO:0071357)

Regulation of viral genome replication (GO:0045069)

Regulation of ubiquitin-protein ligase activity in mitotic cell cycle (GO:0051439)

Tumor necrosis factor-mediated signaling pathway (GO:0033209)

Cellular response to interferon-gamma (GO:0071346)

Regulation of cell cycle (GO:0051726)

Interferon-gamma-mediated signaling pathway (GO:0060333)

Regulation of cellular amino acid metabolic process (GO:0006521)

Regulation of hematopoietic stem cell differentiation (GO:1902036)

$0010 \quad 15$

- $\log 10$ (adjusted P-value) b
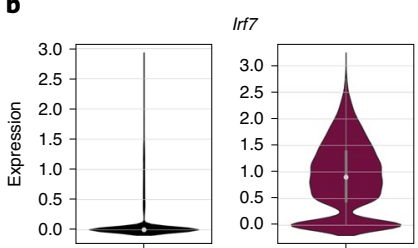

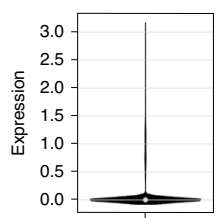

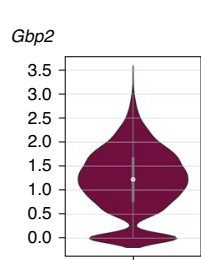

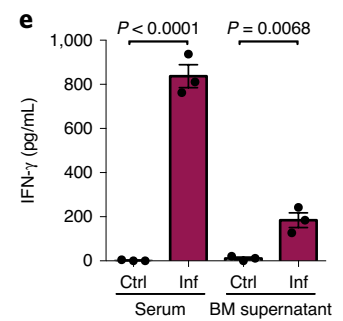

f
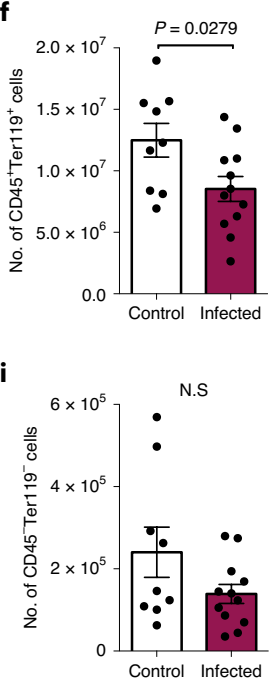

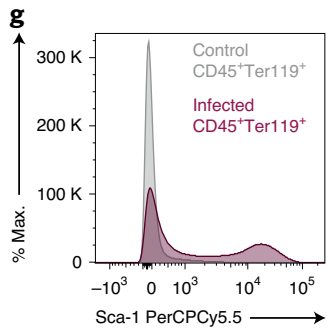

h
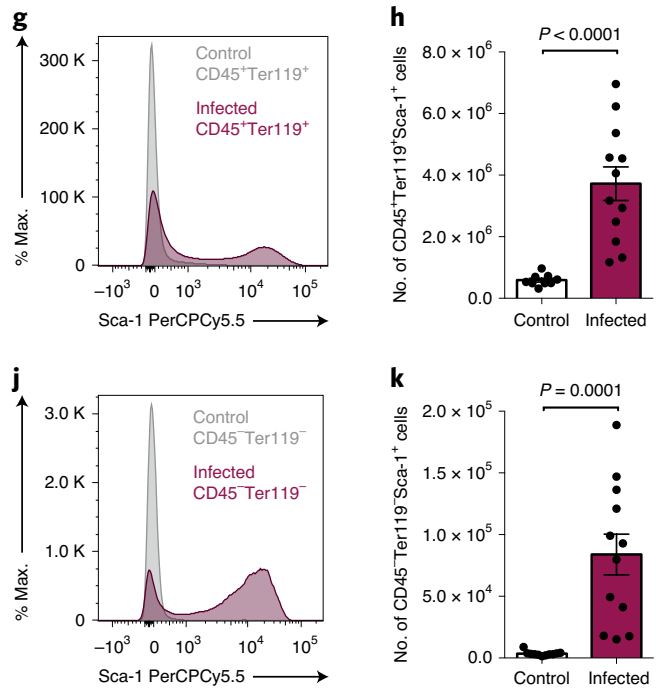

I

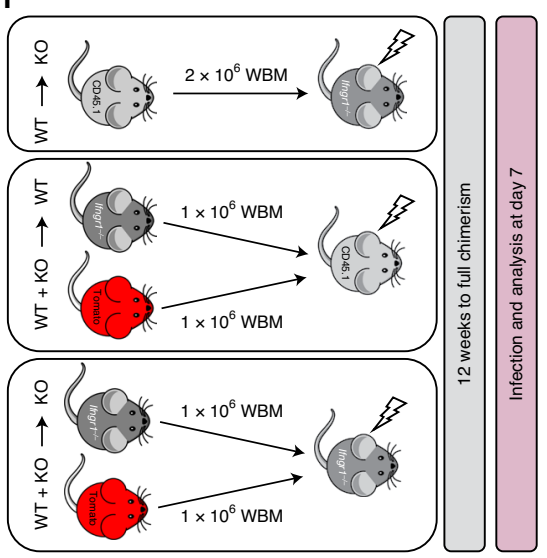

m
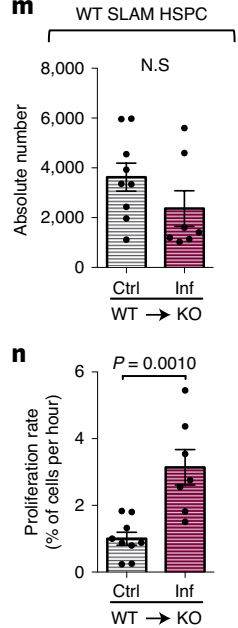
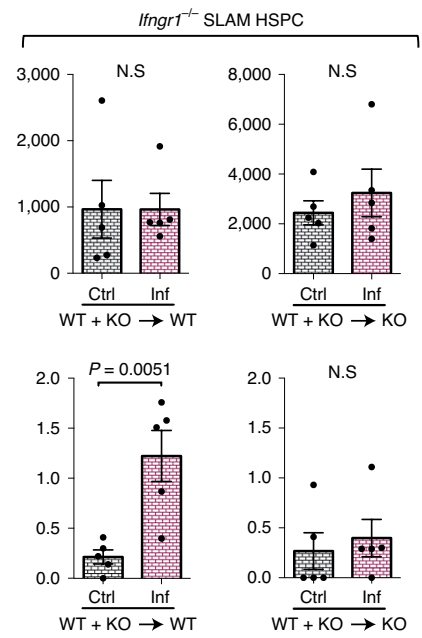
a global reduction of IFN- $\gamma$ levels, the remaining IFN- $\gamma$ may still be sufficient to affect the long-term reconstitution capacity of HSCs exposed to $P$. berghei, possibly by affecting other niche components.

$P$. berghei infection alters the integrity of vessels in the $\mathrm{BM}$ microenvironment. Osteoblasts have been shown to be dependent on healthy endothelial cells ${ }^{45}$ and endothelial cells support HSC maintenance and function ${ }^{46-49}$. Using IVM of Flk1-GFP transgenic mice ${ }^{50}$, we investigated the distribution, morphology and function of $\mathrm{BM}$ endothelial cells. Tilescans revealed no obvious changes in $\mathrm{BM}$ vessels (Fig. 5a) and the number of $\mathrm{GFP}^{+}$voxels remained constant throughout infection (Fig. 5b). Flow cytometry analysis of long-bone endothelial cells, including arteriolar Sca- $1^{+}$and sinusoidal Sca- $1^{-}$endothelial cells, reflected this with no alterations by day 7 psi (Fig. 5c-e).

Time-lapse IVM of randomly selected calvarium BM areas in these mice revealed unusual dynamics over the course of a few hours. While the vasculature of control mice appeared static (Supplementary Video 1), in infected animals, $\mathrm{GFP}^{+}$cells were more dynamic, with many shifting their centre of mass while maintaining their position along the vessel wall (Fig. $5 \mathrm{f}$ and Supplementary Video 1, blue arrowheads) and some separating from the vessel surface and migrating within the extravascular space (Fig. 5f and Supplementary Video 1, red numbered arrowheads). We confirmed by CD45 in vivo staining that these migratory cells were not haematopoietic ${ }^{51}$ (Fig. 5g). Questioning whether the observed $\mathrm{GFP}^{+}$cells may be due to endothelial cell proliferation, flow cytometry analysis revealed no change in the proportion of EdU ${ }^{+}$endothelial cells by day 7 psi (Fig. $5 \mathrm{~h}$ ).

To assess whether the dynamism shown by endothelial cells was an indication of vascular stress, we investigated whether damage developed during the course of $P$. berghei infection, using vascular leakiness as our readout ${ }^{52}$. Following the injection of tetramethylrhodamine (TRITC)-labelled dextran, time-lapse imaging revealed that infected mice had highly permeable vessels (Fig. 5i,j and Supplementary Video 2). HSCs localized in the proximity of more leaky vessels have been shown to have higher levels of reactive oxygen species $(\mathrm{ROS})^{53}$. Thus, we measured intracellular ROS in SLAM HSPCs from infected animals and, as expected, the increased vascular leakiness triggered by $P$. berghei was associated with a twofold rise in the concentration of ROS (Fig. 5k).

PTH treatment coupled with ROS scavenging rescues the function of $P$. berghei-exposed HSCs. Our data on increased vascular leakiness and SLAM HSPC ROS suggest that they may be, among other things, one of the mechanisms leading to loss of functional HSCs during $P$. berghei infection. In line with this, PTH-treated infected animals exhibited leaky vasculature and increased $\mathrm{ROS}^{+}$ SLAM HSPCs (Fig. 6a,b). Vascular permeability is important in enabling immune responses globally; however, ROS are highly damaging to cells. We reasoned that ROS may be an appropriate target to curb HSPC proliferation and/or damage in response to $P$. berghei infection. We treated mice with the ROS scavenger $\mathrm{N}$-acetyl-L-cysteine (NAC) daily from $2 \mathrm{~d}$ before infection until analysis (Fig. 6c). This had no effect on parasitaemia (Extended Data Fig. 7a) but reduced ROS in MPPs and SLAM HSPCs compared with PBS-treated infected animals (Fig. 6d,f). However, proliferation of both cell types was comparable to that of PBS-treated infected controls (Fig. 6e,g). After NAC treatment, vessels remained highly permeable (Fig. 6h), osteoblasts were lost and the absolute number of CD48 ${ }^{\text {neg }}$ HSCs was as reduced as in PBS-treated infected mice (Fig. 6i). Consistent with this, IFN- $\gamma$ levels remained elevated (Fig. 6j). These observations suggest that NAC treatment alone is insufficient to limit HSC and niche damage resulting from $P$. berghei infection.

We hypothesized that a combination of PTH treatment (to reduce proliferation) and NAC treatment (to reduce ROS levels) could have a positive impact on HSC function. Mice were pre-treated with PTH for 2 weeks before infection and NAC for $2 \mathrm{~d}$ before infection, and both treatments continued until analysis (Fig. 7a). Parasitaemia was not affected by treatment (Extended Data Fig. 7b), but ROS levels in both MPPs and SLAM HSPCs were reduced compared with PBS-treated infected controls (Fig. 7b,d). Consistent with PTH treatment alone, PTH + NAC treatment did not affect the proliferation of MPPs (Fig. 7c); however, it reduced the proportion of proliferating SLAM HSPCs compared with PBS-treated infected controls (Fig. 7e). To determine whether this combined treatment could rescue HSC function, we transplanted SLAM HSPCs from each group into lethally irradiated recipient mice. Cells from control, healthy mice achieved the highest engraftment. However, PTH + NAC treatment led to better engraftment of SLAM HSPCs (Fig. 7f) compared with the same cells from PBS-treated infected donors, a lineage output similar to that of healthy donors (Extended Data Fig. 7c), and donors had notably more CD48 ${ }^{\text {neg }}$ HSCs in the BM (Fig. 7g). Interestingly, local and systemic levels of IFN- $\gamma$ in combination-treated mice were strikingly lower than in PBS-treated infected controls (Fig. 7h,i) and suggest, consistent with the scRNA-seq Gene Ontology analysis, that IFN- $\gamma$ is not the only trigger of HSC damage in P. berghei-infected mice. Altogether, our data indicate that, by targeting cellular or chemical components of the HSC niche, it is possible to limit HSC loss during severe infection.

\section{Discussion}

HSC function is profoundly affected by severe infection, but the cellular and molecular mechanisms are not yet fully understood. Here, we used a natural Plasmodium infection to gain a quantitative understanding of its impact on primitive HSPC populations and to investigate the role of the $\mathrm{BM}$ microenvironment in mediating the phenotypes observed.

Fig. 4 | Osteolineage targeting inhibits $\boldsymbol{P}$. berghei-induced HSC proliferation. $\mathbf{a}, \mathbf{b}$, Representative maximum projections and automated segmentation (a) and volume calculation (voxels) (b) of Col2.3-GFP+ osteoblastic cells in tilescans of calvarium BM from control ( $n=4$ ) and infected Col2.3-GFP mice at days $3(n=3), 5(n=3)$ and 7 psi $(n=7)$. c, Schematic of the PTH treatment regimen. d,e, Representative maximum projections (d) and quantification (e) of Col2.3-GFP+ osteoblastic cells in tilescans from PBS- ( $n=5$ controls; $n=4$ infected) and PTH-treated mice ( $n=4$ controls; $n=4$ infected) at day 7 psi. f, Proportion of osteoblasts identified by flow cytometry analysis of long bones from PBS- and PTH-treated mice at day 7 psi ( $n=6$ per group). $\mathbf{g}$, $\mathbf{h}$, Absolute number $(\mathbf{g})$ and proliferation rate $(\mathbf{h})$ of MPPs and SLAM HSPCs in PBS- and PTH-treated mice at day 7 psi. $n$ numbers represent individual mice and are indicated as data points on each graph. i, Peripheral blood reconstitution of transplanted SLAM HSPCs from PBS- and PTH-treated infected mT/ mG donors into lethally irradiated CD45.2 recipient mice, as assessed by flow cytometry ( $n=6$ mice per group). $\mathbf{j}$, Absolute number of CD48neg HSCs in PTH-treated mice at day 7 psi $(n=12$ controls; $n=15$ infected mice). $\mathbf{k}, \mathbf{I}$, IFN- $\gamma$ levels, as measured by ELISA, in BM supernatant (k) and serum (I) from PBS- ( $n=4$ controls; $n=4$ infected) and PTH-treated mice ( $n=6$ controls; $n=6$ infected). m, Absolute number of CD3 ${ }^{+}$T cells in BM from PBS- and PTH-treated mice. $\mathbf{n}-\mathbf{p}, \mathrm{CD} 4^{+}$and $\mathrm{CD} 8^{+}$T cells as a proportion of CD3 ${ }^{+}$T cells $(\mathbf{n})$ and as a proportion of CD4 ${ }^{+}$and $\mathrm{CD} 8^{+} \mathrm{T}$ cells producing IFN- $\gamma(\mathbf{p})$, and CD $44^{+}$PD $-1^{+}$cells as a proportion of activated CD8 ${ }^{+}$T cells $(\mathbf{o})$ in the BM of PBS- and PTH-treated mice, as quantified by flow cytometry ( $n=4$ controls; $n=5$ infected mice). In $\mathbf{m}-\mathbf{p}$, the data were pooled from up to six independent infection experiments. All of the data are presented as means \pm s.e.m. The Pvalues were determined by one-way ANOVA ( $\mathbf{b}, \mathbf{e}, \mathbf{f}, \mathbf{k}-\mathbf{m}$ and $\mathbf{o}$ ) with post-hoc Bonferroni correction, unpaired two-tailed Student's $t$-test ( $\mathbf{g}$-j) or two-way ANOVA (n and p) with post-hoc Bonferroni correction. Scale bars, $500 \mu \mathrm{m}$. 
scRNA-seq analysis of Lineage ${ }^{-} \mathrm{c}-\mathrm{Kit}^{+}$cells provided an unbiased approach and confirmed the development of emergency myelopoiesis described for multiple infections $s^{5,54,55}$. Moreover, we identified a dramatic loss of the most primitive HSPC transcriptional signature and, consistent with this, transplantation assays highlighted infection-induced loss of functional HSCs. Building on a

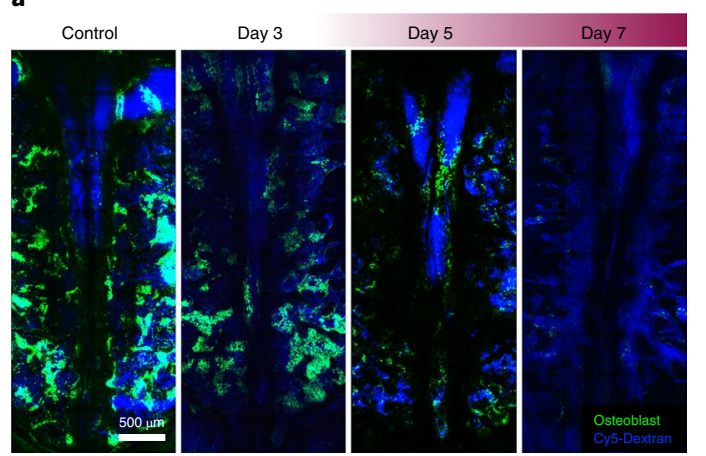

d

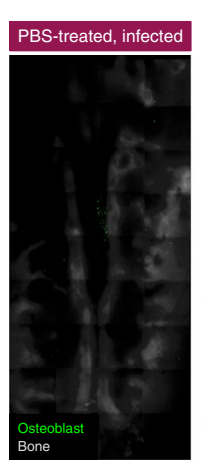

g
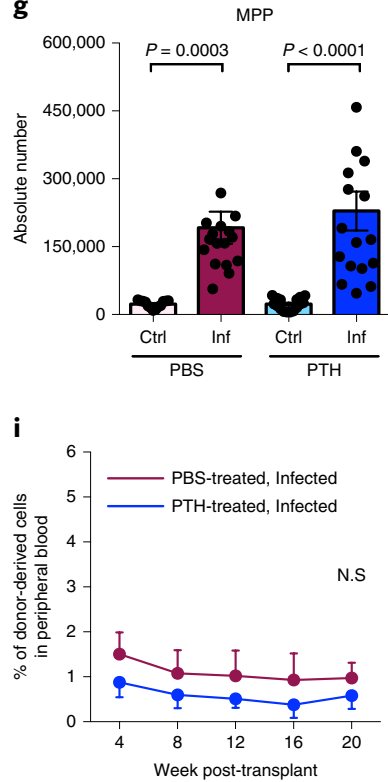

m

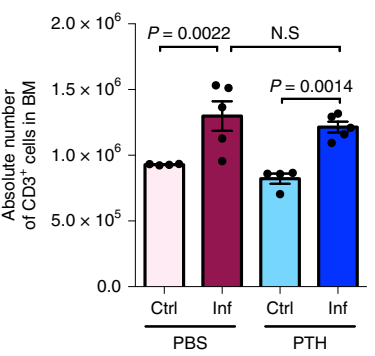

e
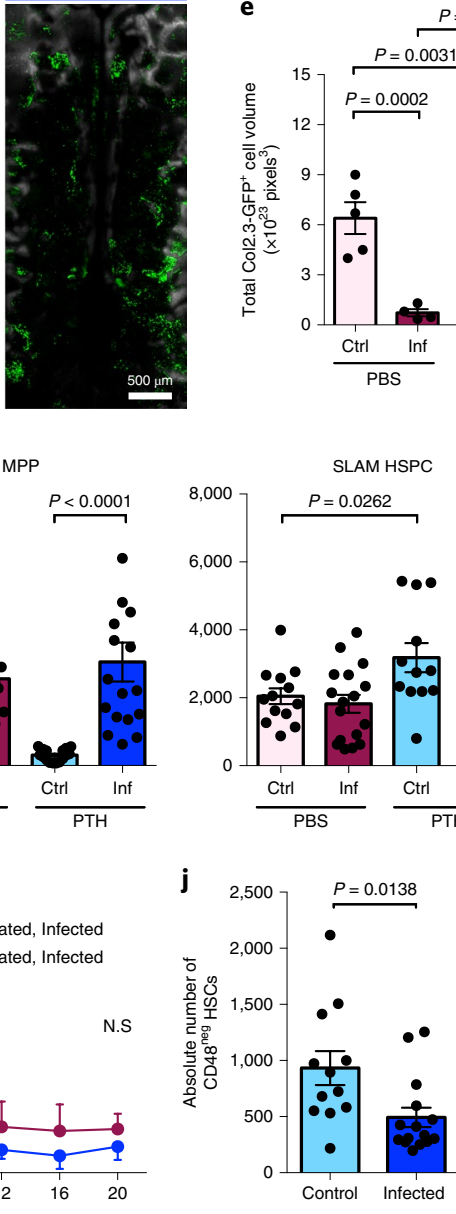
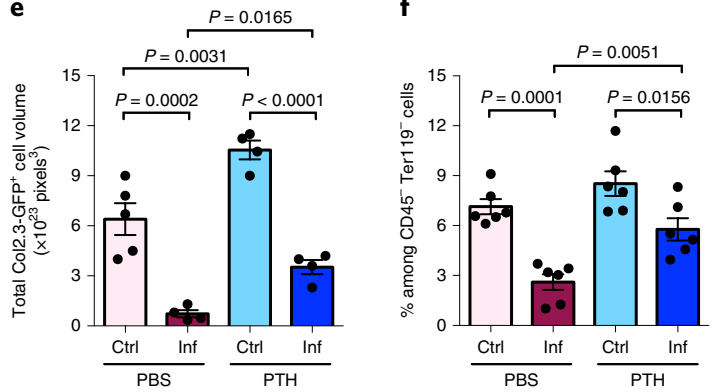

h
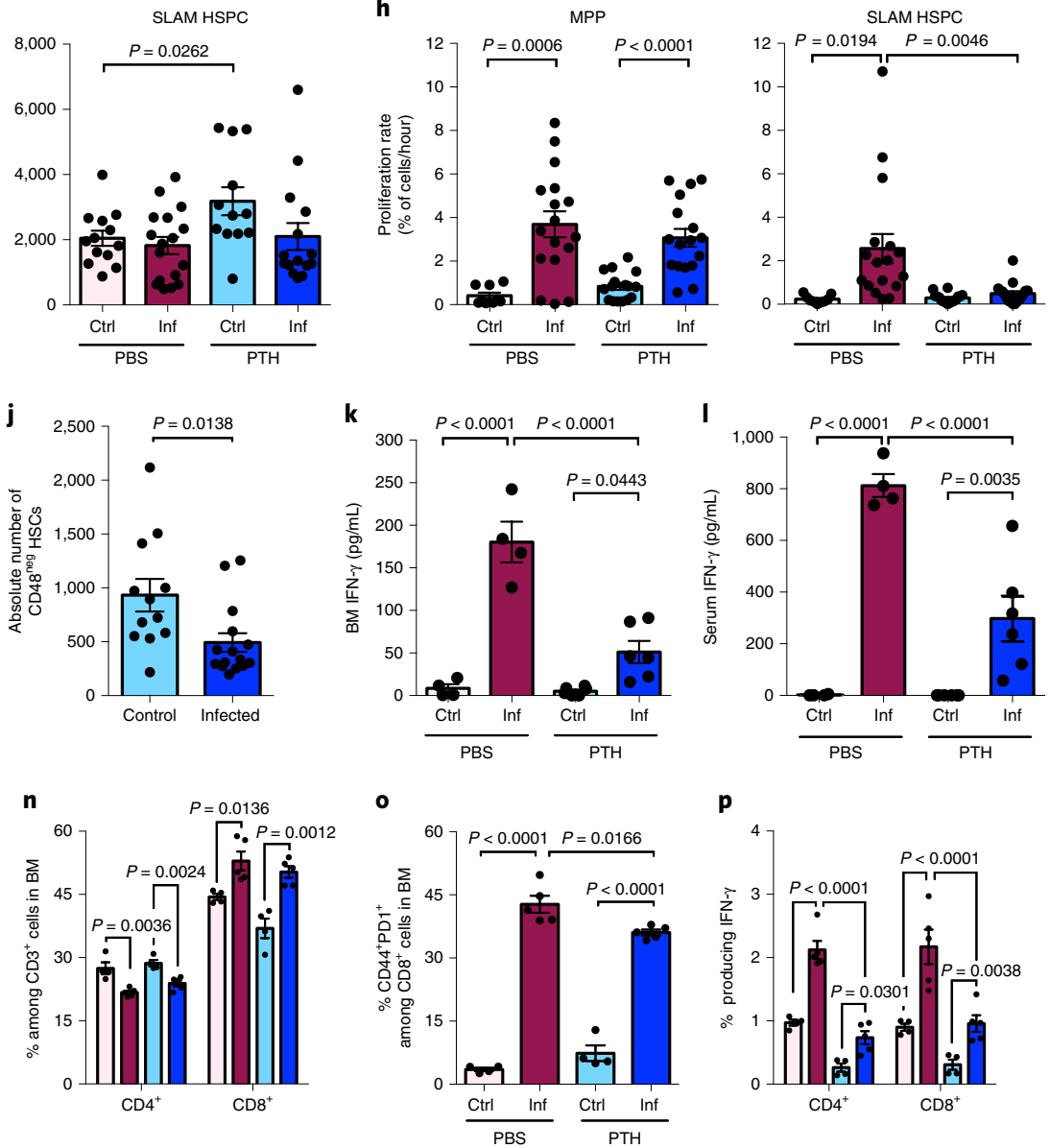
a
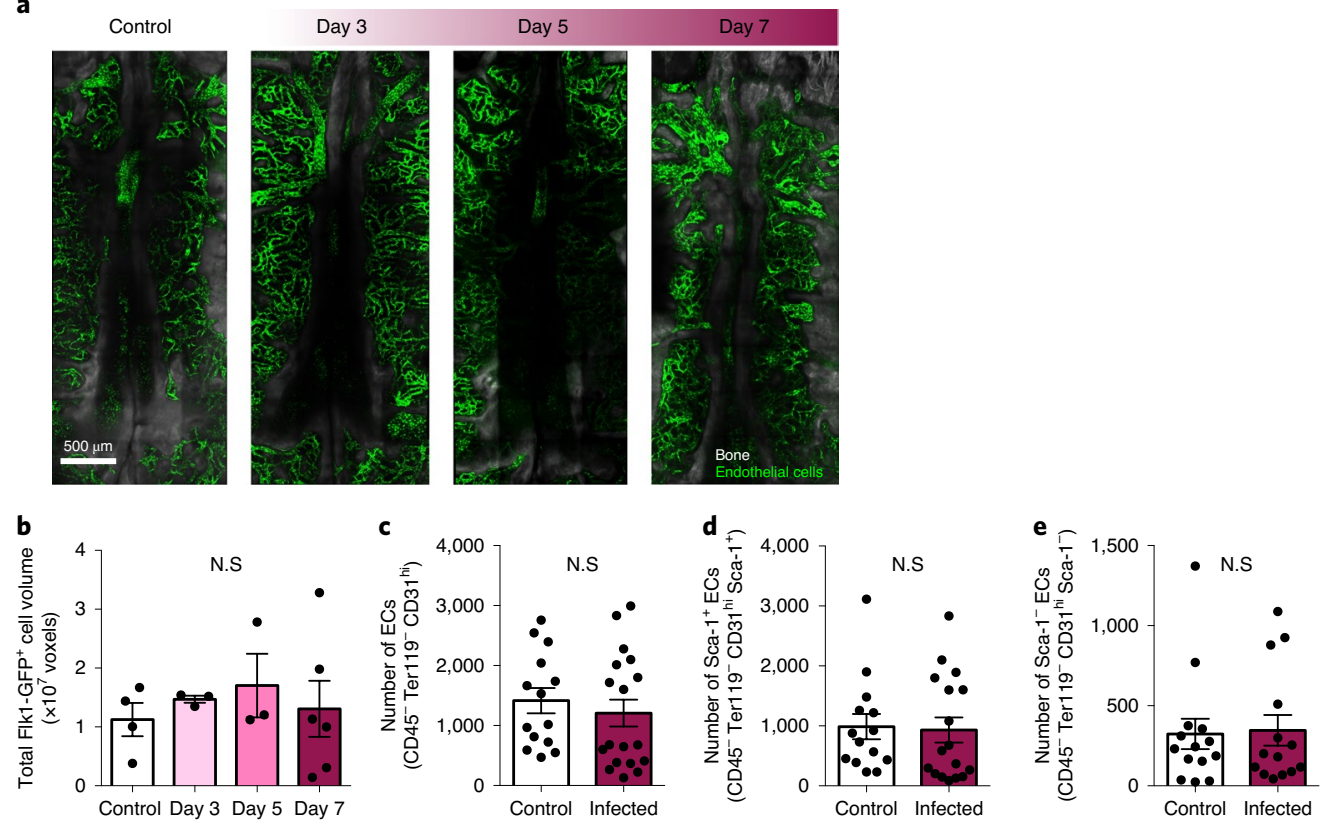

$\mathbf{f}$

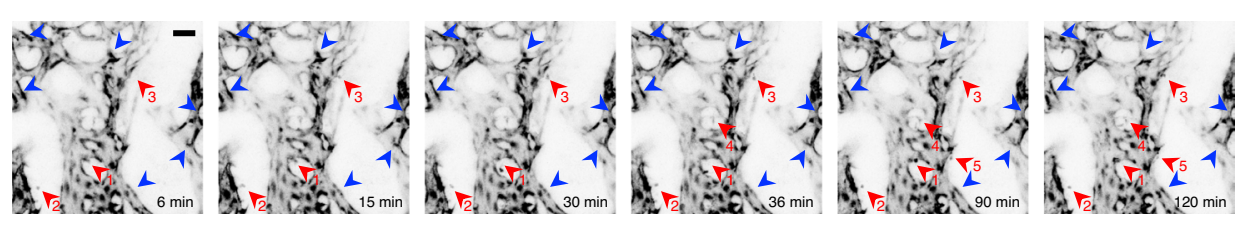

$\mathbf{g}$
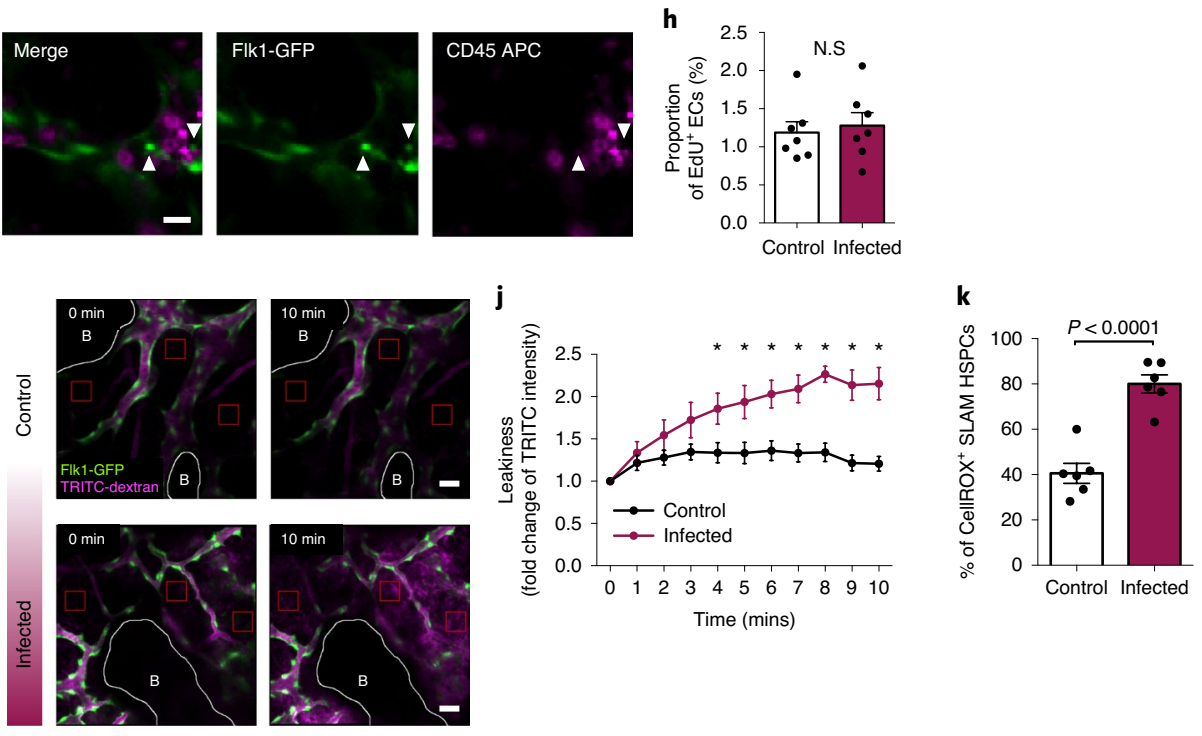

Fig. 5 | P. berghei infection affects vascular integrity and function. $\mathbf{a}, \mathbf{b}$, Representative tilescan maximum projections and automated segmentation (a) and volume calculation (voxels) (b) of Flk1-GFP+ endothelial cells in calvarium BM from control $(n=4)$ and infected Flk1-GFP mice at days 3 ( $n=3$ ), 5 ( $n=3$ ) and 7 psi $(n=6)$. c-e, Absolute numbers of total endothelial cells (ECs) (c), arteriolar Sca- $1^{+}$endothelial cells (d) and sinusoidal Sca- $1^{-}$endothelial cells (e) in control and infected mice at day 7 psi, as analysed by flow cytometry. $n$ numbers represent individual mice and are indicated as data points on the graphs. f, Representative frames from time-lapse imaging of an infected Flk1-GFP mouse, demonstrating a shift in the centre of mass of endothelial cells (blue arrowheads) and detachment and migration of GFP+ cells into the parenchyma (red; numbered arrowheads) at day 7 psi. $\mathbf{g}$, Representative maximum projection of an area of calvarium BM in an infected Flk1-GFP mouse, at day 7 psi, injected with CD45 antibody. The white arrowheads indicate GFP+ cells that have detached from the endothelium. $\mathbf{h}$, Proportion of EdU+ endothelial cells at day 7 psi, as measured by flow cytometry ( $n=7$ controls; $n=7$ infected mice). i, Vascular leakiness was assessed by time-lapse imaging of selected regions (red boxes) within the calvarium following administration of TRITCdextran. Selected frames from control and infected Flk1-GFP mice at day 7 psi demonstrate how extravasation of vascular dye over time allows quantification of vascular permeability. The white lines delineate bone (B). j, Quantification of the fold change of TRITC-dextran intensity in calvaria at day 7 psi $(n=4$ controls; $n=5$ infected mice; $n=4$ positions acquired per mouse). k, Intracellular ROS levels in SLAM HSPCs, as measured by flow cytometry, at day 7 psi ( $n=6$ controls; $n=6$ infected mice). The data were pooled from up to six independent infection experiments. All of the data are presented as means \pm s.e.m. The $P$ values were determined by one-way ANOVA with post-hoc Bonferroni correction (b) or unpaired two-tailed Student's $t$-test (c-e, h, j and $\mathbf{k}$ ), with (j) or without Holm-Šídák correction for multiple comparisons (c-e, h and $\mathbf{k})$. In $\mathbf{j},{ }^{\star} P<0.05$. Scale bars, $500 \mu \mathrm{m}(\mathbf{a}), 50 \mu \mathrm{m}(\mathbf{f}), 20 \mu \mathrm{m}(\mathbf{g})$ and $50 \mu \mathrm{m}(\mathbf{i})$. 
a

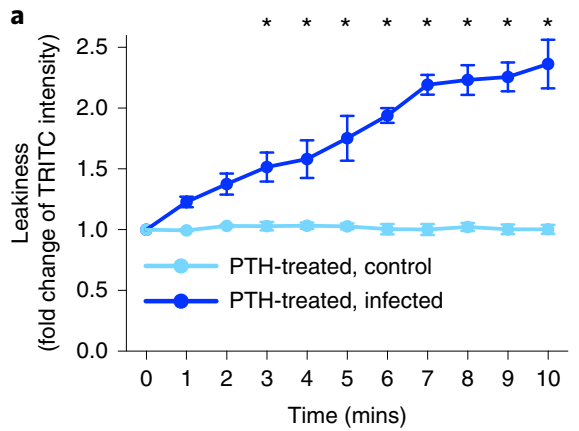

b

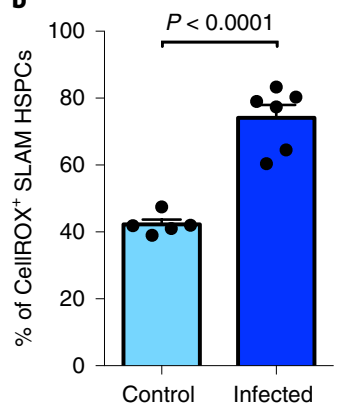

C

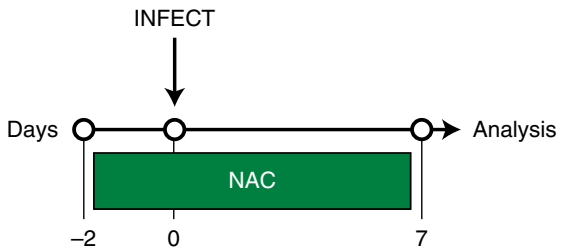

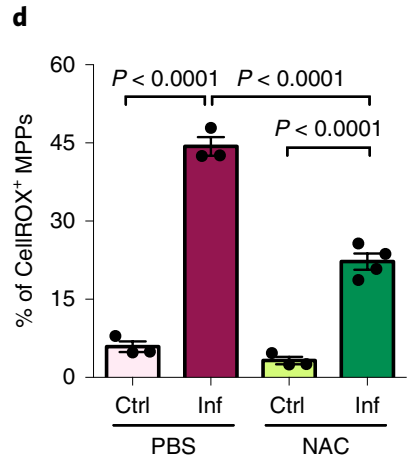

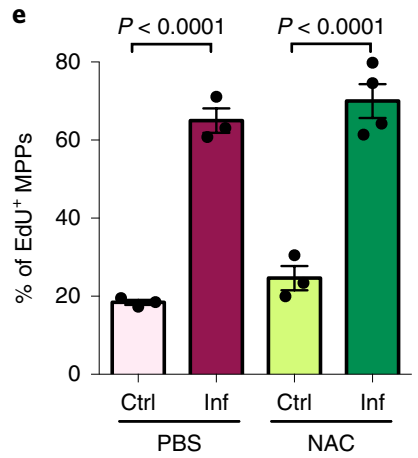

$\mathbf{f}$
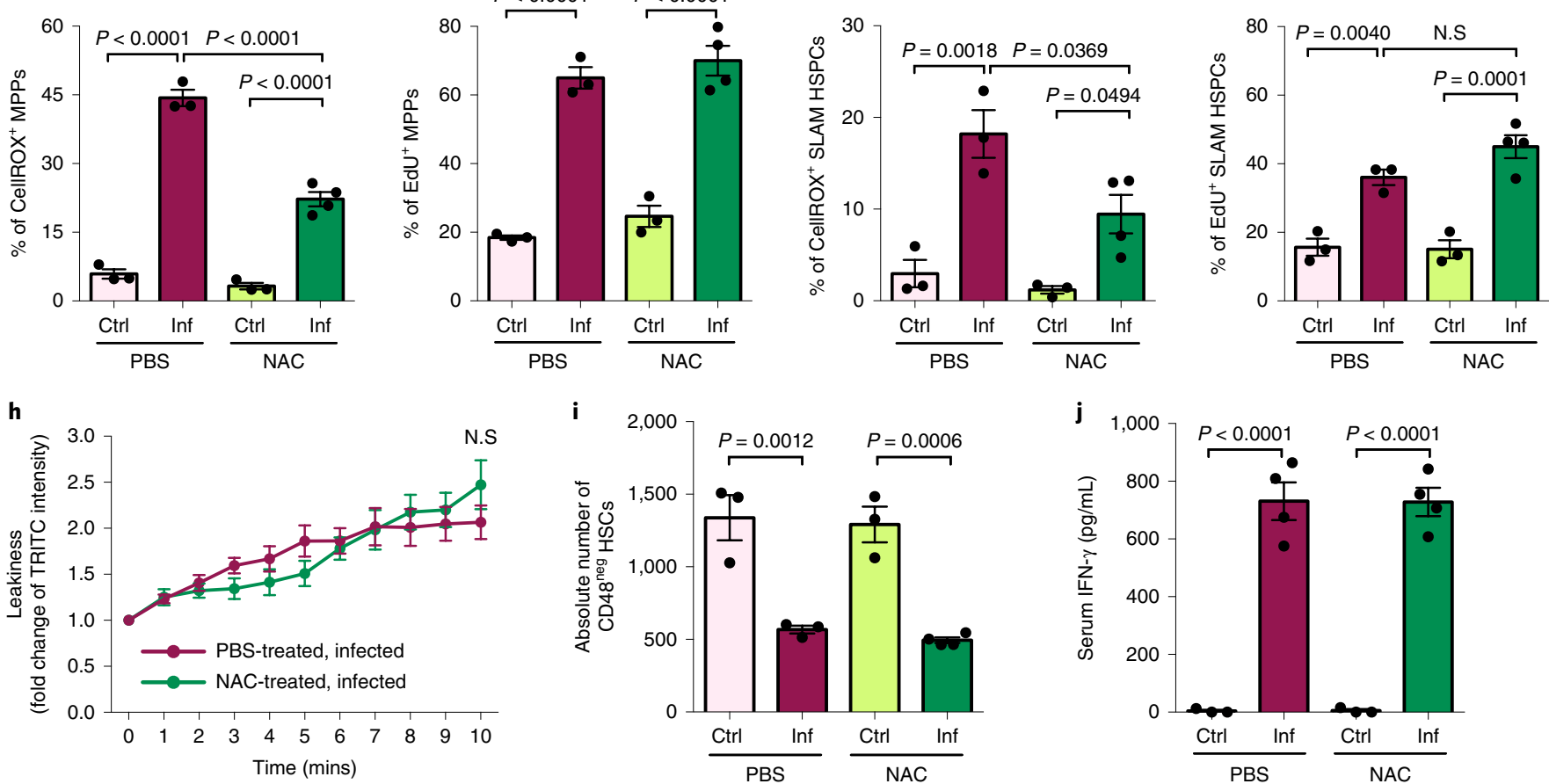

Fig. 6 | Treating infected animals with NAC reduces HSPC intracellular ROS levels. a, Quantification of the fold change of TRITC-dextran intensity measured in the calvaria of PTH-treated control $(n=5)$ and infected $(n=6)$ mice at day 7 psi. b, Cellular ROS levels in SLAM HSPCs, as measured by flow cytometry. in PTH-treated control $(n=5)$ and infected $(n=6)$ mice at day 7 psi. c, Schematic of the NAC treatment regimen carried out. d,f, Cellular ROS levels in MPPs (d) and SLAM HSPCs (f), as measured by flow cytometry in PBS- and NAC-treated control and infected mice at day 7 psi. e,g, Proportion of EdU+ MPPs (e) and SLAM HSPCs (g) in PBS- and NAC-treated control and infected mice at day 7 psi, as measured by flow cytometry. In $\mathbf{d}-\mathbf{g}$, $n$ numbers represent individual mice and are indicated as data points on the graphs. $\mathbf{h}$, Quantification of the fold change of TRITC-dextran intensity measured in the calvaria of PBS- and NAC-treated control $(n=3)$ and infected mice $(n=3)$ at day 7 psi. i, Absolute number of CD48neg HSCs in PBS- and NAC-treated control and infected mice at day 7 psi, as analysed by flow cytometry. $n$ numbers represent individual mice and are indicated as data points on the graph. j, IFN- $\gamma$ levels, as measured by ELISA, in the serum of PBS- and NAC-treated control $(n=3)$ and infected mice $(n=4)$ at day 7 psi. The data were pooled from up to two independent infection experiments. All of the data are presented as means \pm s.e.m. The $P$ values were determined by unpaired two-tailed Student's t-test ( $\mathbf{a}, \mathbf{b}$ and $\mathbf{h}$ ), with ( $\mathbf{a}$ and $\mathbf{h}$ ) or without Holm-Šídák correction for multiple comparisons (b), or one-way ANOVA with post-hoc Bonferroni correction ( $\mathbf{d}-\mathbf{g}$, $\mathbf{i}$ and $\mathbf{j})$. In $\mathbf{a},{ }^{\star} P<0.05$.

our previous qualitative observations ${ }^{9}$, we measured the proliferation rate and compartment size of multiple HSPC populations, and a simple mathematical model demonstrated the extent of increased turnover of each population studied. Both SLAM HSPC and MPP populations turned over multiple times, and even the most quiescent $\mathrm{CD} 48^{\text {neg }}$ HSC compartment was seen to proliferate, albeit at a much lower rate. This suggests that other mechanisms beyond simply proliferation could be driving the dramatic loss of functional HSCs.

Gene expression profiling highlighted the central role of IFN in driving the observed phenotypes. IFN- $\gamma$ is highly expressed in response to multiple strains of human and murine Plasmodium and contributes to controlling the intensity of infection ${ }^{25,26,28}$. However, it has also been reported to mediate and exacerbate the disease itself, causing damage to the host ${ }^{27}$. In our model, the IFN response was shared across not only haematopoietic cells but also BM stroma, including known components of the HSC niche. Consistent with a role of the $\mathrm{BM}$ microenvironment in mediating infection and IFN- $\gamma$-driven HSC damage, Ifngr $1^{-/-}$SLAM HSPCs in chimeric mice with haematopoietic cells derived from both wild-type and Ifngr $1^{-/-}$donors exhibited a proliferative response. Importantly, this was lost when the stroma was Ifngrl deficient, indicating that $P$. berghei-induced IFN- $\gamma$ affects HSPCs both directly and indirectly, through niche cells that sense and respond to it. 

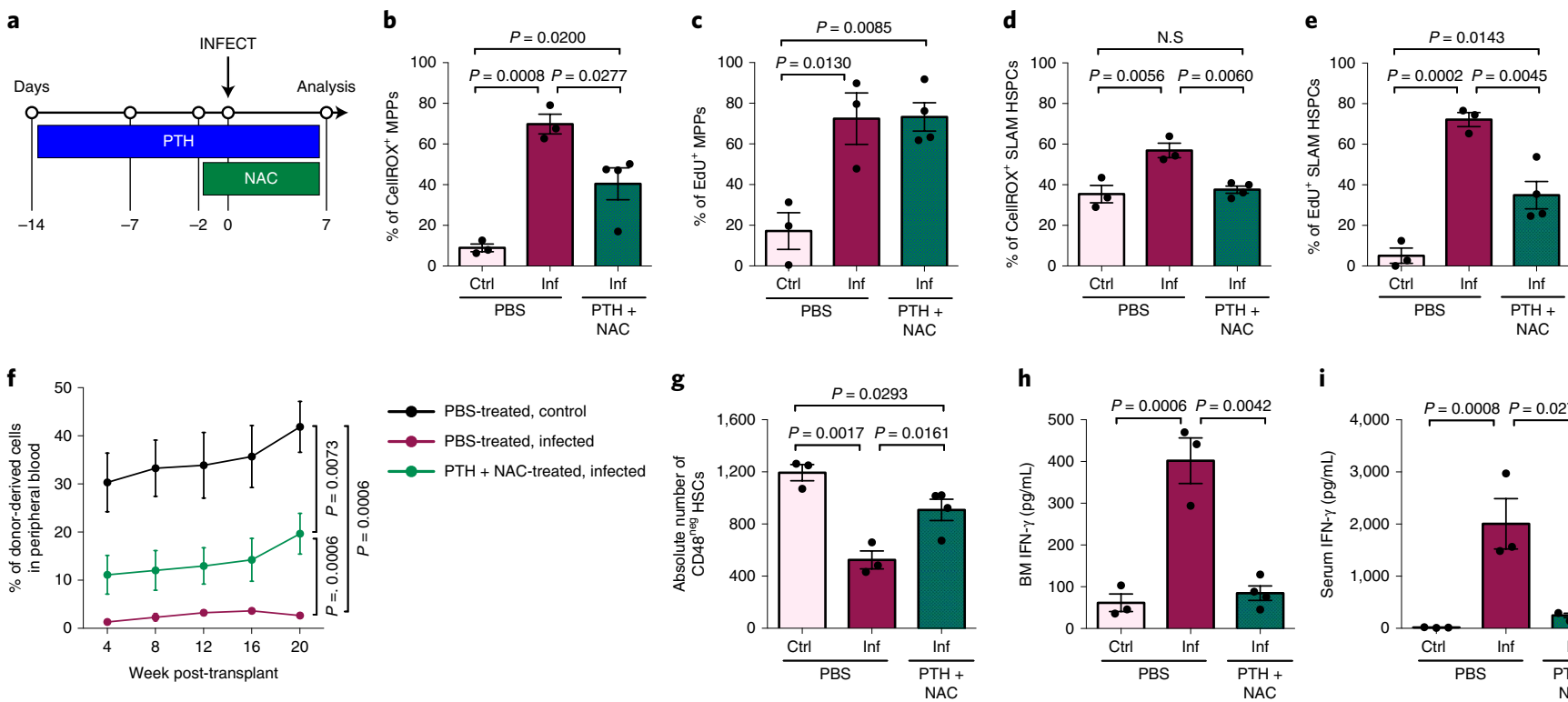

h

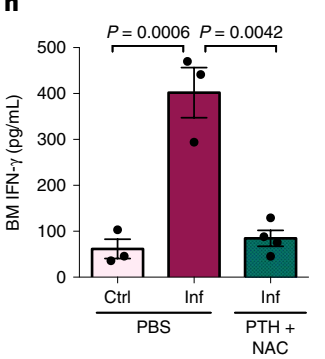

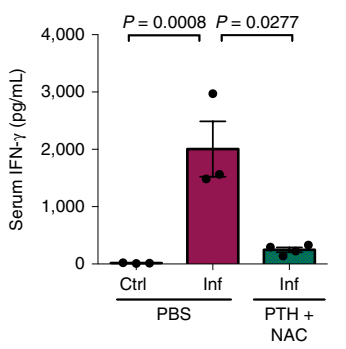

Fig. 7 | Combining PTH treatment and sequestration of ROS during infection partially rescues HSC function. a, Schematic of the PTH and NAC combined treatment regimen carried out. b,d, Cellular ROS levels in MPPs (b) and SLAM HSPCs (d), as measured by flow cytometry in PBS- and PTH + NAC-treated control and infected mice at day 7 psi. $\mathbf{c}, \mathbf{e}$, Proportion of EdU+ MPPs (c) and SLAM HSPCs (e) in PBS- and PTH + NAC-treated control and infected mice at day 7 psi, as measured by flow cytometry. In $\mathbf{b}-\mathbf{e}, n$ numbers represent individual mice and are indicated as data points on the graphs. $\mathbf{f}$, Peripheral blood reconstitution of transplanted SLAM HSPCs from PBS-treated control (black line), PBS-treated infected (maroon line) and PTH + NAC-treated infected CD45.2 donors (green line) into lethally irradiated $\mathrm{mT} / \mathrm{mG}$ recipient mice, as assessed by flow cytometry of tomato- cells up to 20 weeks after transplantation ( $n=7$ recipients of PBS-treated control cells; $n=6$ recipients of PBS-treated infected cells; $n=7$ recipients of PTH + NAC-treated infected cells). $\mathbf{g}$, Absolute number of CD48 neg HSCs in PBS-treated control $(n=3)$ and infected mice $(n=3)$ and PTH + NAC-treated infected mice $(n=4)$ at day $7 \mathrm{psi}$, as analysed by flow cytometry. $\mathbf{h}, \mathbf{i}$, IFN- $\gamma$ levels, as measured by ELISA, in the BM supernatant (h) and serum (i) of PBS-treated control $(n=3)$ and infected mice $(n=3)$ and PTH + NAC-treated infected mice $(n=4)$ at day 7 psi. The data were pooled from up to two independent infection experiments. All of the data are presented as means \pm s.e.m. The $P$ values were determined by one-way ANOVA with post-hoc Bonferroni correction (b-e and $\mathbf{g}-\mathbf{i})$ or exact one-tailed permutation test (f).

It was recently reported that both Plasmodium infection and sepsis affect bone biology ${ }^{5,57}$. Sepsis-induced loss of osteoblasts has been linked to the loss of lymphoid progenitors and myeloid-skewed haematopoiesis, and the same mechanism is likely to take place in response to Plasmodium infection. Here, we show that loss of osteoblasts is specifically associated with primitive HSPC proliferation and loss of function, potentially through a reduction of the most HSC-enriched cell populations, including CD48 ${ }^{\text {neg }} \mathrm{HSCs}$. Osteoblast maintenance through PTH treatment was associated with the rescue of infection-induced HSC proliferation but did not preserve HSC function. This is an important finding as HSC proliferation and loss of function are usually directly linked ${ }^{5,8}$. While the effects seen on mature osteoblasts are dramatic, we cannot exclude an important role for other mesenchymal cells contributing to the effects of PTH; however, based on our data, it is unlikely that HSC proliferation is regulated directly by PTH-responsive T cells.

$B M$ vascular damage was recently reported in response to IFN- $\alpha^{58}$ and in mice burdened with malaria, where it was proposed to mediate parasite accumulation in the tissue $e^{59,60}$. Our data on vascular leakiness and HSPC intracellular ROS are consistent with previous observations that steady-state HSCs residing in the vicinity of more permeable vessels contain higher levels of ROS and are less functional ${ }^{53}$, suggesting that increased vascular permeability may be one of the mechanisms leading to loss of functional HSCs during P. berghei infection, and the same is likely to happen in human malaria. ROS trigger signalling cascades and are a recognized component of the HSC niche chemical milieu ${ }^{61}$. ROS quenching alone did not rescue vascular leakiness, nor SLAM HSPC proliferation or osteoblasts loss. However, combined PTH treatment and ROS quenching partially reduced SLAM HSPC proliferation and limited HSC function decline. PTH + NAC-treated mice displayed IFN- $\gamma$ levels similar to those of uninfected animals, suggesting that PTH + NAC treatment does not impair immune responses and that IFN- $\gamma$ is an important trigger of HSC damage, albeit not the only one. Given the effects of PTH and NAC treatments alone, we propose that the two interventions combined may act side by side and on specific components of the HSC milieu; however, further studies will be needed to fully understand the interplay of all of the involved cell types within the BM. While our interventions are not directly translatable to malaria sufferers, our data provide a promising indication that it might prove possible to limit HSC damage during severe infection, and future work should focus on identifying therapeutic approaches aimed at preserving HSCs through the course of severe infections.

\section{Online content}

Any methods, additional references, Nature Research reporting summaries, source data, extended data, supplementary information, acknowledgements, peer review information; details of author contributions and competing interests; and statements of data and code availability are available at https://doi.org/10.1038/ s41556-020-00601-w.

Received: 3 July 2019; Accepted: 6 October 2020;

Published online: 23 November 2020

\section{References}

1. Morrison, S. J. \& Scadden, D. T. The bone marrow niche for haematopoietic stem cells. Nature 505, 327-334 (2014). 
2. Essers, M. A. G. et al. IFN $\alpha$ activates dormant haematopoietic stem cells in vivo. Nature 458, 904-908 (2009).

3. Esplin, B. L. et al. Chronic exposure to a TLR ligand injures hematopoietic stem cells. J. Immunol. 186, 5367-5375 (2011).

4. King, K. Y. \& Goodell, M. A.Inflammatory modulation of HSCs: viewing the HSC as a foundation for the immune response. Nat. Rev. Immunol. 11, 685-692 (2011).

5. MacNamara, K. C., Jones, M., Martin, O. \& Winslow, G. M. Transient activation of hematopoietic stem and progenitor cells by IFN $\gamma$ during acute bacterial infection. PLoS ONE 6, e28669 (2011)

6. Baldridge, M. T., King, K. Y., Boles, N. C., Weksberg, D. C. \& Goodell, M. A. Quiescent haematopoietic stem cells are activated by IFN- $\gamma$ in response to chronic infection. Nature 465, 793-797 (2010).

7. Rashidi, N. M. et al. In vivo time-lapse imaging shows diverse niche engagement by quiescent and naturally activated hematopoietic stem cells. Blood 124, 79-83 (2014).

8. Matatall, K. A. et al. Chronic infection depletes hematopoietic stem cells through stress-induced terminal differentiation. Cell Rep. 17, 2584-2595 (2016).

9. Vainieri, M. L. et al. Systematic tracking of altered haematopoiesis during sporozoite-mediated malaria development reveals multiple response points. Open Biol. 6, 160038 (2016).

10. Phillips, M. A. et al. Malaria. Nat. Rev. Dis. Prim. 3, 17050 (2017).

11. Dörmer, P., Dietrich, M., Kern, P. \& Horstmann, R. Ineffective erythropoiesis in acute human P. falciparum malaria. Blut 46, 279-288 (1983).

12. Maggio-Price, L., Brookoff, D. \& Weiss, L. Changes in hematopoietic stem cells in bone marrow of mice with Plasmodium berghei malaria. Blood 66, 1080-1085 (1985).

13. Wickramasinghe, S., Looareesuwan, S., Nagachinta, B. \& White, N. Dyserythropoiesis and ineffective erythropoiesis in Plasmodium vivax malaria. Br. J. Haematol. 72, 91-99 (1989).

14. Boehm, D., Healy, L., Ring, S. \& Bell, A. Inhibition of ex vivo erythropoiesis by secreted and haemozoin-associated Plasmodium falciparum products. Parasitology 145, 1865-1875 (2018).

15. Orf, K. \& Cunnington, A. J. Infection-related hemolysis and susceptibility to Gram-negative bacterial co-infection. Front. Microbiol. 6, 666 (2015).

16. White, N. J. Anaemia and malaria. Malar. J. 17, 371 (2018).

17. Mamedov, M. R. et al. A macrophage colony-stimulating-factor-producing $\gamma \delta \mathrm{T}$ cell subset prevents malarial parasitemic recurrence. Immunity 48, 350-363.e7 (2018)

18. Weinreb, C., Wolock, S. \& Klein, A. SPRING: a kinetic interface for visualizing high dimensional single-cell expression data. Bioinform. Oxf. Engl. https://doi.org/10.1093/bioinformatics/btx792 (2017).

19. Hamey, F. K. \& Göttgens, B. Machine learning predicts putative haematopoietic stem cells within large single-cell transcriptomics datasets. Exp. Hematol. 78, 11-20 (2019).

20. Wilson, A. et al. Hematopoietic stem cells reversibly switch from dormancy to self-renewal during homeostasis and repair. Cell 135, 1118-1129 (2008).

21. Oguro, H., Ding, L. \& Morrison, S. J. SLAM family markers resolve functionally distinct subpopulations of hematopoietic stem cells and multipotent progenitors. Cell Stem Cell 13, 102-116 (2013).

22. Cabezas-Wallscheid, N. et al. Identification of regulatory networks in HSCs and their immediate progeny via integrated proteome, transcriptome, and DNA methylome analysis. Cell Stem Cell 15, 507-522 (2014).

23. Akinduro, O. et al. Proliferation dynamics of acute myeloid leukaemia and haematopoietic progenitors competing for bone marrow space. Nat. Commun. 9, 519 (2018).

24. Kent, D. G. et al. Prospective isolation and molecular characterization of hematopoietic stem cells with durable self-renewal potential. Blood 113, 6342-6350 (2009)

25. Meding, S., Cheng, S., Simon-Haarhaus, B. \& Langhorne, J. Role of gamma interferon during infection with Plasmodium chabaudi chabaudi. Infect. Immun. 58, 3671-3678 (1990).

26. John, C. C. et al. Gamma interferon responses to Plasmodium falciparum liver-stage antigen 1 and thrombospondin-related adhesive protein and their relationship to age, transmission intensity, and protection against malaria. Infect. Immun. 72, 5135-5142 (2004).

27. King, T. \& Lamb, T. Interferon- $\gamma$ : the Jekyll and Hyde of malaria. PLoS Pathog 11, e1005118 (2015).

28. Lelliott, P. M. \& Coban, C. IFN- $\gamma$ protects hepatocytes against Plasmodium vivax infection via LAP-like degradation of sporozoites. Proc. Natl Acad. Sci. USA 113, 6813-6815 (2016)

29. Morales-Mantilla, D. E. \& King, K. Y. The role of interferon-gamma in hematopoietic stem cell development, homeostasis, and disease. Curr. Stem Cell Rep. 4, 264-271 (2018).

30. Ma, X., Ling, K. \& Dzierzak, E. Cloning of the $L y-6 A$ (Sca-1) gene locus and identification of a $3^{\prime}$ distal fragment responsible for high-level $\gamma$-interferoninduced expression in vitro. Br. J. Haematol. 114, 724-730 (2001).
31. Boulais, P. E. et al. The majority of $\mathrm{CD} 45^{-}$Ter $119^{-} \mathrm{CD} 31^{-}$bone marrow cell fraction is of hematopoietic origin and contains erythroid and lymphoid progenitors. Immunity 49, 627-639.e6 (2018).

32. Calvi, L. M. et al. Osteoblastic cells regulate the haematopoietic stem cell niche. Nature 425, 841-846 (2003).

33. Zhang, J. et al. Identification of the haematopoietic stem cell niche and control of the niche size. Nature 425, 836-841 (2003).

34. Arai, F. et al. Tie2/angiopoietin-1 signaling regulates hematopoietic stem cell quiescence in the bone marrow niche. Cell 118, 149-161 (2004).

35. Lo Celso, C. et al. Live-animal tracking of individual haematopoietic stem/ progenitor cells in their niche. Nature 457, 92-96 (2009).

36. Kalajzic, I. et al. Use of type I collagen green fluorescent protein transgenes to identify subpopulations of cells at different stages of the osteoblast lineage. J. Bone Miner. Res. 17, 15-25 (2002).

37. Jilka, R. L. et al. Increased bone formation by prevention of osteoblast apoptosis with parathyroid hormone. J. Clin. Invest. 104, 439-446 (1999).

38. Jilka, R. L. Molecular and cellular mechanisms of the anabolic effect of intermittent PTH. Bone 40, 1434-1446 (2007).

39. Jilka, R. L. et al. Intermittent PTH stimulates periosteal bone formation by actions on post-mitotic preosteoblasts. Bone 44, 275-286 (2009).

40. Kim, S. W. et al. Intermittent parathyroid hormone administration converts quiescent lining cells to active osteoblasts. J. Bone Miner. Res. 27, 2075-2084 (2012).

41. Balani, D. H., Ono, N. \& Kronenberg, H. M. Parathyroid hormone regulates fates of murine osteoblast precursors in vivo. J. Clin. Invest. 127, 3327-3338 (2017)

42. Li, J.-Y. et al. PTH expands short-term murine hemopoietic stem cells through T cells. Blood 120, 4352-4362 (2012).

43. Yao, H. et al. Parathyroid hormone enhances hematopoietic expansion via upregulation of cadherin-11 in bone marrow mesenchymal stromal cells. Stem Cells 32, 2245-2255 (2014).

44. Terauchi, M. et al. T lymphocytes amplify the anabolic activity of parathyroid hormone through Wnt10b signaling. Cell Metab. 10, 229-240 (2009).

45. Duarte, D. et al. Inhibition of endosteal vascular niche remodeling rescues hematopoietic stem cell loss in AML. Cell Stem Cell 22, 64-77.e6 (2018).

46. Hooper, A. T. et al. Engraftment and reconstitution of hematopoiesis is dependent on VEGFR2-mediated regeneration of sinusoidal endothelial cells. Cell Stem Cell 4, 263-274 (2009).

47. Butler, J. M. et al. Endothelial cells are essential for the self-renewal and repopulation of notch-dependent hematopoietic stem cells. Cell Stem Cell 6, 251-264 (2010)

48. Kobayashi, H. et al. Angiocrine factors from Akt-activated endothelial cells balance self-renewal and differentiation of haematopoietic stem cells. Nat. Cell Biol. 12, 1046-1056 (2010).

49. Winkler, I. G. et al. Vascular niche E-selectin regulates hematopoietic stem cell dormancy, self renewal and chemoresistance. Nat. Med. 18, 1651-1657 (2012).

50. Ishitobi, H. et al. Flk1-GFP BAC Tg mice: an animal model for the study of blood vessel development. Exp. Anim. Tokyo 59, 615-622 (2010).

51. Kirito, K., Fox, N., Komatsu, N. \& Kaushansky, K. Thrombopoietin enhances expression of vascular endothelial growth factor (VEGF) in primitive hematopoietic cells through induction of HIF-1 $\alpha$. Blood 105, 4258-4263 (2005).

52. Passaro, D. et al. Increased vascular permeability in the bone marrow microenvironment contributes to disease progression and drug response in acute myeloid leukemia. Cancer Cell 32, 324-341.e6 (2017).

53. Itkin, T. et al. Distinct bone marrow blood vessels differentially regulate haematopoiesis. Nature 532, 323-328 (2016).

54. Boettcher, S. et al. Cutting Edge: LPS-induced emergency myelopoiesis depends on TLR4-expressing nonhematopoietic cells. J. Immunol. 188, 5824-5828 (2012).

55. Schürch, C. M., Riether, C. \& Ochsenbein, A. F. Cytotoxic CD8 ${ }^{+}$T cells stimulate hematopoietic progenitors by promoting cytokine release from bone marrow mesenchymal stromal cells. Cell Stem Cell 14, 460-472 (2014)

56. Lee, M. S. et al. Plasmodium products persist in the bone marrow and promote chronic bone loss. Sci. Immunol. 2, eaam8093 (2017).

57. Terashima, A. et al. Sepsis-induced osteoblast ablation causes immunodeficiency. Immunity 44, 1434-1443 (2016).

58. Prendergast, Á. M. et al. IFN $\alpha$-mediated remodeling of endothelial cells in the bone marrow niche. Haematologica 102, 445-453 (2016).

59. Niz, M. D. et al. Plasmodium gametocytes display homing and vascular transmigration in the host bone marrow. Sci. Adv. 4, eaat3775 (2018).

60. Joice, R. et al. Plasmodium falciparum transmission stages accumulate in the human bone marrow. Sci. Transl. Med. 6, 244re5 (2014).

61. Batsivari, A. et al. Dynamic responses of the haematopoietic stem cell niche to diverse stresses. Nat. Cell Biol. 22, 7-17 (2020).

Publisher's note Springer Nature remains neutral with regard to jurisdictional claims in published maps and institutional affiliations.

(c) The Author(s), under exclusive licence to Springer Nature Limited 2020 


\section{Methods}

Mice. The animal work was carried out in accordance with the animal ethics committee (Animal Welfare Ethical Review Body) at Imperial College London and UK Home Office regulations (Animals (Scientific Procedures) Act, 1986) under licence numbers 70/8403 and PP9504146. The Flk1-GFP ${ }^{50}$ mice were a gift from A. Medvinsky (University of Edinburgh). The Tuck CD1 and C57BL/6 mice were obtained from Envigo or Charles River. The Col2.3-GFP ${ }^{36}, \mathrm{mT} / \mathrm{mG}^{62}$ and CD45.1 (B6/SJL) mice were bred and housed at Imperial College London or the Sir Francis Crick Institute, according to institutional guidelines. The Ifngr $1^{-/-63}$ mice were kindly provided by J. Langhorne (Sir Francis Crick Institute). Male and female mice $>6$ weeks of age were used in all of the experiments. The mice were housed in Tecniplast mouse greenline cages with appropriate bedding and enrichment. The temperature, humidity and light cycles were kept within the UK Home Office code of practice, with the temperature between 20 and $24^{\circ} \mathrm{C}$, the room humidity at $45-65 \%$ and a $12 \mathrm{~h} / 12 \mathrm{~h}$ light cycle with a 30 -min dawn and dusk period to provide a gradual change.

For PTH treatment, rat PTH (1-34) (Bachem; $80 \mu \mathrm{gg}^{-1}$ body weight) or PBS (vehicle control) was injected intraperitoneally five times a week for 2 weeks before the infection and for a further week through infection until sacrifice and analysis. Strictly, 4- to 6-week-old female mice were used. To determine the effects of PTH treatment post-infection, PTH treatment was initiated on the day of infection and administered daily until analysis at day 7 psi.

For NAC treatment, NAC (Sigma-Aldrich; $130 \mu \mathrm{g} \mathrm{kg}^{-1}$ body weight) or PBS (vehicle control) was injected intraperitoneally daily, starting $2 \mathrm{~d}$ before infection and throughout until sacrifice and analysis.

For combined treatment with PTH + NAC, mice received PTH five times a week for 2 weeks and NAC for $2 \mathrm{~d}$ before infection, and both treatments carried on through infection until sacrifice and analysis.

P. berghei experimental model. Parasite maintenance, the generation of infected mosquitos and the infection of mice were carried out as previously described ${ }^{9,64}$. Briefly, red fluorescent protein-tagged $P$. berghei ${ }^{65}$ was maintained in 4 - to 10 -week-old female Tuck CD1 mice by serial blood passage, according to Home Office-approved protocols. Hyper-reticulocytosis was induced 2-3d before infection by treating with $6 \mathrm{mg} \mathrm{ml}^{-1}$ phenylhydrazine chloride. Stock mice were infected by intraperitoneal injection of blood containing parasites, and parasitaemia was monitored on Giemsa-stained tail blood smears and expressed as a percentage of more than 500 red blood cells counted per slide. For each individual experiment, a group of five female CD1 phenylhydrazine chloride-treated mice were infected with intraperitoneally injected $P$. berghei, followed by feeding to mosquitos. Infected mice were subsequently anaesthetized and exposed to cages containing 500 starved female Anopheles stephensi mosquitos. Fed mosquitos were maintained until $21 \mathrm{~d}$ post-infection, at which point salivary gland sporozoites are at their peak. To infect mice with $P$. berghei for the experiments, each mouse was exposed to five infected mosquitos, with control mice receiving five mosquito bites from naive, non-infected mosquitos in parallel. Blood parasitaemia and symptoms of malaria were monitored, and mice were sacrificed for analysis at time points of interest (always before the onset of cerebral malaria).

Transplantation assays. Tibias, femurs, ileac bones, vertebrae and sternum were harvested from control or infected (untreated or PBS-, PTH- or $\mathrm{PTH}+\mathrm{NAC}$-treated) $\mathrm{mT} / \mathrm{mG}, \mathrm{CD} 45.1$ (B6/SJL) or CD45.2 (C57BL/6) donor mice at day 7 psi. The bones were crushed and filtered through a $40-\mu \mathrm{m}$ strainer, and red blood cells were lysed. WBM was labelled with a cocktail of biotinylated lineage antibodies (CD3, CD4, CD8, Ter119, B220, Ly6G and CD11b) and streptavidin magnetic MicroBeads (Miltenyi Biotech) to perform a lineage depletion using the MACS Column Technology (Miltenyi Biotech). The lineage-depleted sample was stained and sorted for SLAM HSPCs (defined as Lineage ${ }^{-} \mathrm{c}-\mathrm{Kit}^{+} \mathrm{Sca}-$ $\left.1^{+} \mathrm{CD} 150^{+} \mathrm{CD} 48^{\text {neg/ow }}\right)$ on a FACSAria III (BD Biosciences). Some 200 SLAM HSPCs were transplanted into lethally irradiated (two doses of $5.5 \mathrm{~Gy}$, at least $3 \mathrm{~h}$ apart) CD45.2 (when testing CD45.1 or $\mathrm{mT} / \mathrm{mG}$ HSPCs) or $\mathrm{mT} / \mathrm{mG}$ recipients (when testing CD45.2 HSPCs) alongside 300,000 support BM cells from a donor matching the recipients' genotype. Baytril antibiotic was administered to recipient mice for 6 weeks post-transplant. Multilineage engraftment of the recipients was monitored every 4 weeks up to week 20 by flow cytometry analysis of the peripheral blood.

Generation of BM chimeras. Reverse chimeras (wild-type $\mathrm{BM} \rightarrow$ Ifngr $1^{-/}$ recipients and wild type $\rightarrow$ knockout) were generated by transplanting $2 \times 10^{6}$ WBM cells from CD45.1 donor mice into lethally irradiated Ifngr $1^{-/-}$recipients (CD45.2). Mixed BM chimeras were generated by transplanting $1 \times 10^{6} \mathrm{WBM}$ cells from Ifngr ${ }^{-/-}$mice and $1 \times 10^{6} \mathrm{WBM}$ cells from $\mathrm{mT} / \mathrm{mG}$ (CD45.2) mice into lethally irradiated CD45.1 (knockout + wild type $\rightarrow$ wild type) or Ifngr $1^{-/-}$recipient mice (knockout + wild type $\rightarrow$ knockout). Peripheral blood reconstitution was assessed by flow cytometry 8 weeks after transplantation and stable chimeras were infected for experiments at approximately 12 weeks post-transplantation.

Flow cytometry. For the analysis of HSPC and T cell compartments in the BM, bones were crushed in PBS with $2 \%$ foetal bovine serum and the cells were filtered through a 40- $\mu \mathrm{m}$ strainer, depleted of red blood cells and stained with relevant antibodies. For analysis of stroma and endothelial cells, bones were crushed and digested with collagenase I (Worthington) at $37^{\circ} \mathrm{C}$ for $20 \mathrm{~min}$ with 110 r.p.m. agitation. For analysis of osteoblasts, bone chips were digested twice with collagenase I at $37^{\circ} \mathrm{C}$ for $45 \mathrm{~min}$ with agitation. The cells obtained were filtered and stained with relevant antibodies. For analysis of T cell compartments in peripheral blood, approximately $400 \mu \mathrm{l}$ blood was obtained by cardiac puncture and mixed with ethylenediaminetetraacetic acid to prevent clotting. For analysis of reconstitution in peripheral blood during transplantation assays, approximately $10 \mu \mathrm{l}$ blood was obtained from mice via the tail vein. Red blood cells were subsequently lysed and the cells were washed with $2 \%$ foetal bovine serum and then stained with appropriate antibodies. For information on all of the antibodies used, see Supplementary Table 3. BrdU and EdU were detected and analysed as previously described ${ }^{23}$ using a combination of the BrdU kit (Becton Dickinson) and Click-iT EdU Kit (Life Technologies). For the analysis of cellular ROS, CellROX Deep Red Reagent was used, following the manufacturer's instructions (Thermo Fisher Scientific). Calibrite beads (BD Biosciences) were used to determine the absolute cell numbers in the populations of interest, as described previously ${ }^{66}$. Cells were analysed with an LSRFortessa (BD Biosciences) and the data were analysed with FlowJo (Tree Star). Please see Extended Data Figs. 8-10 for additional gating strategies used for analysis of the data presented in this manuscript.

\section{Mathematical modelling. Please see the Supplementary Note for details.}

scRNA-seq and analysis. For the $10 \times$ Chromium ( $10 \times$ Genomics) experiments, two mice were selected based on showing parasitaemia close to average levels from a pool of six mice infected at the same time. Lineage ${ }^{-} \mathrm{c}-\mathrm{Kit}^{+}$cells were sort purified, as described above, from these infected mice and two control, uninfected mice, and the cells were subsequently processed according to the manufacturer's protocol. Libraries were sequenced with a HiSeq 4000 and corresponding Illumina commercial software.

Data processing and quality control. Sample demultiplexing, barcode processing and gene counting were performed using the count commands from the Cell Ranger version 1.3 pipeline. Each sample was filtered for potential doublets by simulating synthetic doublets from pairs of scRNA-seq profiles and assigning scores based on a $k$-nearest-neighbour classifier on principle component analysis-transformed data. The top $4.5 \%$ of cells with the highest doublet scores from each sample were removed from further analysis. Cells with $>10 \%$ of unique molecular identifier counts mapping to mitochondrial genes, expressing fewer than 500 genes, or with the total number of unique molecular identifier counts further than 3 s.d. from the mean for all cells, were excluded.

Identification of variable genes. Variable genes were identified for all samples following the method of Macosko et al..$^{67}$, with expression $=0.001$ and dispersion $=0.05$ used as minimum cut-offs. The cells were normalized to the same total count for each cell and $\log$ transformed $(x \rightarrow \log [x+1])$. Each gene was scaled so it was zero centred. Typically, 5,000 variable genes were carried forward for analysis. All further analysis was performed using the Scanpy Python module. In total, there were 14,193 control and 13,905 infected cells.

Clustering and mapping. The control cells were clustered using Louvain clustering (Scanpy igraph method). To assign infected cells to clusters, the infected samples were projected into the principle component analysis space of the control data and the nearest neighbours were calculated between the control and infected samples based on the Euclidean distance in the top 50 components. Infected cells were assigned to the same cluster that the majority of their 15 nearest control neighbours belonged to. Force graphs for the control and infected datasets were created by constructing $k=7$ nearest neighbour graphs on the top 50 principal components of the variable genes for each dataset. Edge lists were exported into Gephi 0.9.1 and graph coordinates were calculated using the ForceAtlas 2 layout. Data visualization was also carried out in R using the ggplot2 package. MA plots were created by plotting the $\log _{2}$ [fold change] of infected cells against the mean expression in the control expression for each gene. DEGs were identified as those with an adjusted $P$ value of $<0.05$ and a fold change of $>1.5$. The bar graph showing the abundance changes of certain cell types between control and infected cells were created by calculating the percentage abundances of each cell cluster and calculating the $\log _{2}$ [fold change] of the infected cells compared with the control. The infected cells were split into two samples, each corresponding to one mouse.

HSC score. Each cell transcriptome for control and infected samples was scored using the HSC score method ${ }^{19}$. Briefly, the transcriptome of each cell was compared with the transcriptomes of known HSCs using a multilayer perceptron (MLP) deep learning model to assign a score representing how likely the cell was to be a true HSC. These scores were then interpolated between 0 and 1 and plotted on the force graphs.

Determining initial IFN genes, correlating IFN genes and DEGs. Differential expression was performed between the control and infected cells for each cluster. 
This was done in the $\mathrm{R}$ package edge $\mathrm{R}$, using a likelihood ratio test. Genes that were at least fourfold upregulated in the infected compared with control cells in all clusters were chosen as an initial set of driving genes involved in the infection. This list contained 20 genes. The entire set of variable genes (calculated using the combined control and infected samples) was analysed to find all of the genes whose expression correlated highly with any of those in the initial set of 20. Genes that had a Spearman correlation of $>0.3$ with any of these genes were added to the list of driving genes. This procedure added 89 genes for a total of 109 driving genes. All violin plots were generated using the Python modules seaborn or matplotlib with default parameters. In a further, independent analysis, a Wilcoxon rank-sum test with multiple testing correction was used to identify DEGs by comparing the entire control/infected dataset or each control/infected cluster. An adjusted $P$ value threshold of $<0.05$ and a fold change threshold of $>1.5$ were used to calculate the lists of statistically DEGs. Gene Ontology analysis of biological processes was carried out using EnrichR ${ }^{68,69}$.

Enzyme-linked immunosorbent assay (ELISA). To obtain BM supernatants, tibias and femurs were harvested from experimental mice. The metaphysis and diaphysis of long bones were separated with scissors and $80 \mu \mathrm{l}$ PBS was flushed through each diaphysis into an Eppendorf tube, collected and re-flushed through the bone. Cells were pelleted by centrifugation at $400 \mathrm{~g}$ for $5 \mathrm{~min}$. The supernatan was removed and any remaining cells were excluded by further centrifugation at $500 \mathrm{~g}$ for $5 \mathrm{~min}$. Serum was prepared by collecting blood by cardiac puncture after terminal anaesthesia with pentobarbital. The blood was incubated at $4{ }^{\circ} \mathrm{C}$ for at least $3 \mathrm{~h}$ to enable it to clot and subsequently centrifuged at $12,000 \mathrm{~g}$ for $10 \mathrm{~min}$ at $4^{\circ} \mathrm{C}$. The serum supernatant was transferred to an Eppendorf tube. BM supernatants and serum were stored at $-20^{\circ} \mathrm{C}$ until they were required for ELISA. IFN- $\gamma$, TNF- $\alpha$ and IFN- $\alpha$ ELISAs (BioLegend and Invitrogen) were performed according to the manufacturer's instructions, and by diluting BM supernatants $1: 4$ and serum samples $1: 2$.

IVM. IVM was performed using a Zeiss LSM 780 upright confocal microscope equipped with argon $(458,488$ and $514 \mathrm{~nm})$, a diode-pumped solid-state $561-\mathrm{nm}$ laser, a helium-neon $633 \mathrm{~nm}$ laser and a tuneable infrared multiphoton laser, four non-descanned detectors and an internal spectral detector array. Live imaging of the calvarium BM was carried out as described in previously published reports ${ }^{45,70}$. Blood vessels were labelled with $80 \mu \mathrm{l} 8 \mathrm{mg} \mathrm{ml}^{-1}$ Cy5-Dextran (Nanocs) or TRITC-dextran (Sigma-Aldrich). The second harmonic signal was excited at $860-880 \mathrm{~nm}$ and detected with external detectors. The cyan fluorescent protein signal was excited at 870 or $458 \mathrm{~nm}$ and detected using external or internal detectors. the GFP signal was excited at 880 or $488 \mathrm{~nm}$ and detected using external or internal detectors. The Cy5 signal was excited at $633 \mathrm{~nm}$ and detected using internal detectors. Assessment of vascular leakiness with infection was carried out by adapting previously published protocols ${ }^{52,53}$. Briefly, with the mouse ready on the microscope stage and the positions for analysis selected, $60 \mu \mathrm{l}$ low-molecular-weight TRITC-dextran $\left(3 \mathrm{mg} \mathrm{ml}^{-1} ; 65-80 \mathrm{kDa}\right)$ was injected intravenously and the positions were continuously recorded (one frame per minute) for $10 \mathrm{~min}$ after injection.

BM histology. Femurs were harvested from control and infected animals at day $7 \mathrm{psi}$, fixed in $4 \%$ paraformaldehyde at $4{ }^{\circ} \mathrm{C}$ overnight and decalcified in $10 \%$ ethylenediaminetetraacetic acid for $3 \mathrm{~d}$. The bones were subsequently embedded in paraffin, sectioned and processed for haematoxylin and eosin staining at the National Heart and Lung Institute histopathology facility at Imperial College.

Image processing and quantification. Zen black (Zeiss) software was used to stitch three-dimensional BM tilescans (tilescans represent individual tiles stitched together to form a composite). ImageJ was used to visualize, register and process raw data. ImageJ was also used to manually crop out autofluorescent signal from the tissue. Automated cell segmentation and volume measurements were performed in Definiens (Definiens Developer 64). For analysis of TRITC-dextran extravasation in time-lapse videos from control and infected mice, the pixel intensity within three equally sized and randomly placed regions of interest was evaluated per time frame. The average of these values was subsequently calculated and the fold change increase in TRITC intensity in control and infected samples was plotted as a graph.

Statistics and reproducibility. Raw data were visualized and processed using Microsoft Excel and GraphPad Prism (GraphPad Software). Group means were compared using unpaired, two-tailed Student's $t$-tests. For multiple comparisons, unpaired, two-tailed Student's $t$-tests or one- or two-way analysis of variance (ANOVA) with post-hoc Bonferroni or Holm-Šidák corrections were used. For Fig. $7 \mathrm{f}$, an exact one-tailed permutation test was implemented in MATLAB for the time-course data. The statistic used was the sum across days of the difference between the mean reconstitution in the PTH + NAC-treated and PBS-treated infected cohorts, and between the mean reconstitution of PBS-treated infected and control cohorts. For all data, differences were considered significant when $P<0.05$. All specific statistical details can be found in the figure captions. For all experiments, $n$ corresponds to the number of independent experiments providing values for statistical analysis. At least three biological replicates were performed for all in vivo experiments, with similar results obtained. Mice were randomly allocated to control or experimental conditions and experimenters were not blinded to the conditions during data collection or analyses.

Reporting Summary. Further information on research design is available in the Nature Research Reporting Summary linked to this article.

\section{Data availability}

The scRNA-seq data that support the findings of this study have been deposited in the Gene Expression Omnibus under accession code GSE156410. Data are also available in processed form for interactive browsing at http://128.232.224.252/ malaria_control/ and http://128.232.224.252/malaria_infected/. All other data supporting the findings of this study are available from the corresponding author on reasonable request. Source data are provided with this paper.

\section{Code availability}

The computational code used in this study can be obtained by request to B.G. (bg200@cam.ac.uk).

\section{References}

62. Muzumdar, M., Tasic, B., Miyamichi, K., Li, L. \& Luo, L. A global doublefluorescent Cre reporter mouse. Genesis 45, 593-605 (2007).

63. Huang, S. et al. Immune response in mice that lack the interferon-gamma receptor. Science 259, 1742-1745 (1993).

64. Ramakrishnan, C. et al. Laboratory maintenance of rodent malaria parasites. Methods Mol. Biol. 923, 51-72 (2013).

65. Sturm, A. et al. Alteration of the parasite plasma membrane and the parasitophorous vacuole membrane during exo-erythrocytic development of malaria parasites. Protist 160, 51-63 (2009).

66. Hawkins, E. D. et al. Measuring lymphocyte proliferation, survival and differentiation using CFSE time-series data. Nat. Protoc. 2, 2057-2067 (2007).

67. Macosko, E. Z. et al. Highly parallel genome-wide expression profiling of individual cells using nanoliter droplets. Cell 161, 1202-1214 (2015).

68. Chen, E. Y. et al. Enrichr: interactive and collaborative HTML5 gene list enrichment analysis tool. BMC Bioinformatics 14, 128 (2013).

69. Kuleshov, M. V. et al. Enrichr: a comprehensive gene set enrichment analysis web server 2016 update. Nucleic Acids Res. 44, W90-W97 (2016).

70. Hawkins, E. D. et al. T-cell acute leukaemia exhibits dynamic interactions with bone marrow microenvironments. Nature 538, 518-522 (2016).

\section{Acknowledgements}

This work was funded by the ERC, BBSRC and Wellcome Trust (ERC_STG 337066, BB/L023776/1 and IA 212304/Z/18/Z to C.L.C.; PhD studentship 105398/Z/14/Z to M.L.R.H.). A.M.B. was funded by the MRC (NIRG MR/N00227X/1). S.W. was funded by an MRC studentship. T.C.L. was funded by a Sir Henry Dale Fellowship (210424/Z/18/Z). Work by the Göttgens group is funded by the Wellcome Trust, Bloodwise and Cancer Research UK, with core funding from the Wellcome-MRC Cambridge Stem Cell Institute. C.L.C. and K.R.D. were supported in part by the Royal Irish Academy-Royal Society International Exchange Program (IEC $\backslash R 1 \backslash 180061)$. We thank M. Tunnicliff for passaging parasites and preparing mosquitos, F. Angrisano and K. Sala for technical assistance and advice with infections, I. Kucinski for support creating the interactive website, Imperial College DoLS Flow Cytometry and Central Biomedical Services and Crick Biological Research Facilities for support, and H. Fletcher, A. Cunnington and all of the Lo Celso group members for constructive discussions.

\section{Author contributions}

M.L.R.H., C.L.C., A.M.B. and R.E.S. conceived of the project. A.M.B. provided all of the mosquitos and support with infections. M.L.R.H. conducted the core experiments and data analysis. K.E., A.L., H.A., F.B., C.P., S.G.A. and N.R. performed the animal and flow cytometry experiments. S.W., N.K.W. and B.G. implemented the scRNA-seq and analysis. K.R.D. conducted the mathematical modelling. M.L.V. created the histological sections. C.P., T.C.L. and J.L. contributed to the experimental design and data analysis. M.L.R.H. and C.L.C. analysed the data and wrote the manuscript. All authors contributed to feedback.

\section{Competing interests}

The authors declare no competing interests.

\section{Additional information}

Extended data is available for this paper at https://doi.org/10.1038/s41556-020-00601-w.

Supplementary information is available for this paper at https://doi.org/10.1038/ s41556-020-00601-w.

Correspondence and requests for materials should be addressed to C.L.

Reprints and permissions information is available at www.nature.com/reprints. 
a

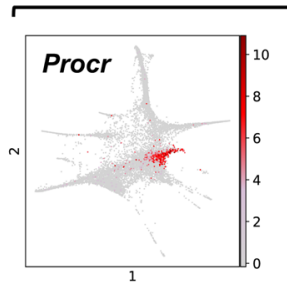

Primitive HSPC

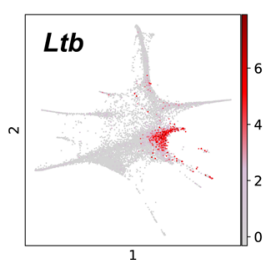

b

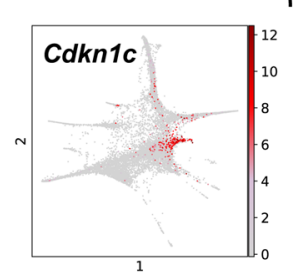

Myeloid

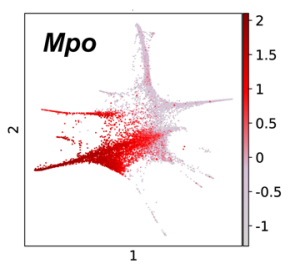

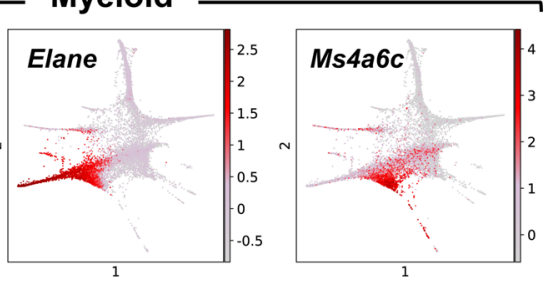

d

Erythroid

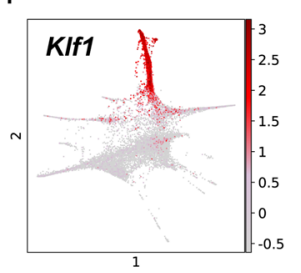

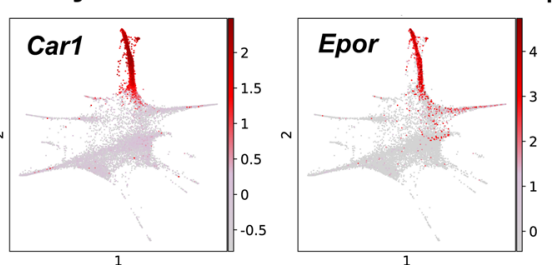

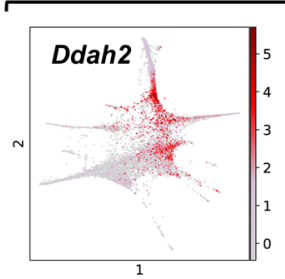

MEP

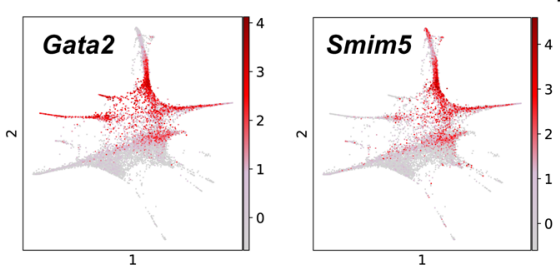

f

Megakaryocyte

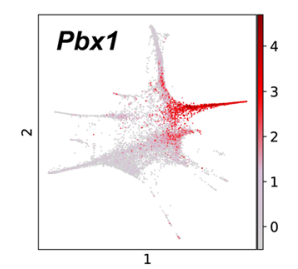

Basophil

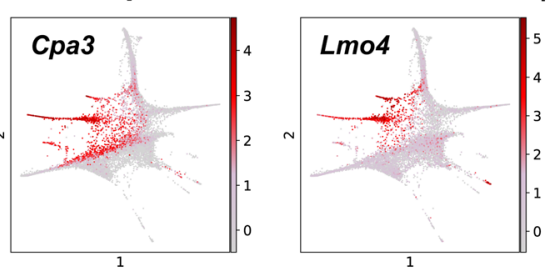

g

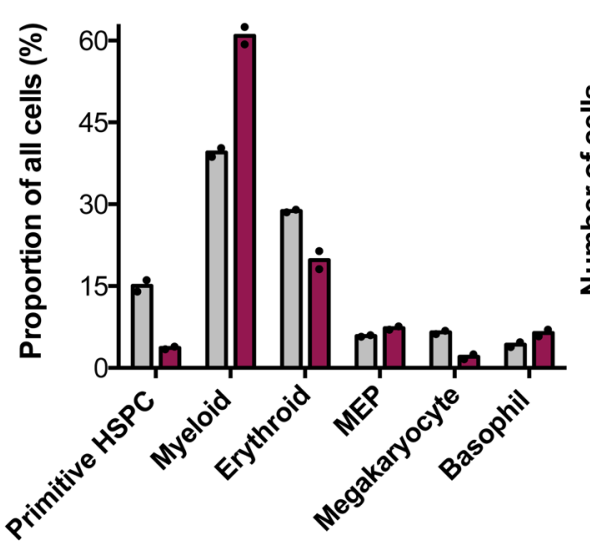

h

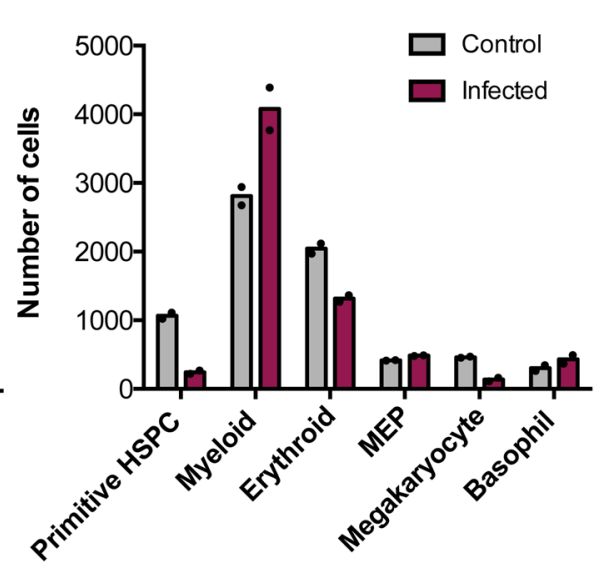

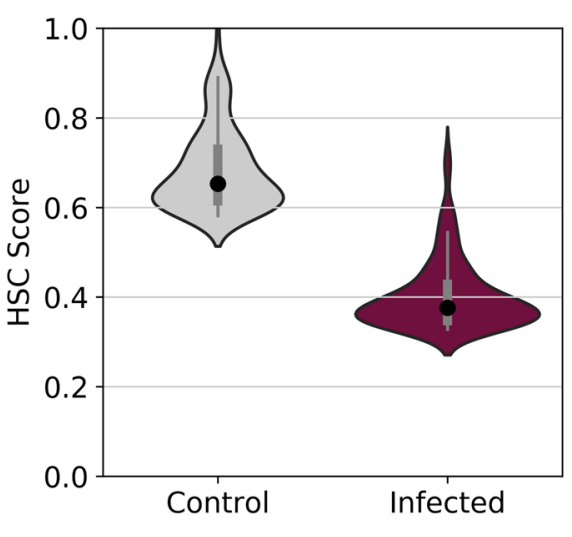

Extended Data Fig. 1 | Transcriptomic analysis reveals rewiring of haematopoiesis and loss of HSC identity in response to $P$. berghei infection. The expression of some exemplar genes used to determine the clustering of $\mathbf{a}$, primitive HSPC, b, myeloid, $\mathbf{c}$, erythroid, $\mathbf{d}$, MEP, $\mathbf{e}$, megakaryocyte and $\mathbf{f}$, basophil cell populations plotted on the force-directed graph embedding for cells from control and infected mice at day 7 psi. $\mathbf{g}$, Proportion (left panel) and number of cells (right panel) in each cluster shown in Fig. 1c. Each dot represents one mouse and bars represent the mean ( $n=2$ control, 2 infected mice). $\mathbf{h}$, Violin plots quantifying HSC-scores calculated for the top $1 \%$ of highest scoring cells in the control and infected samples based on the fact that multi-potential HSCs occur on the order of approximately 1 out of every 100 cells found in the Lineage ${ }^{-}$-Kit $^{+}$gate. $n=142$ control cells and 140 infected cells. The centre dot shows the median and the box limits show the upper and lower quartiles. The whiskers extend from the box limit to the smallest and largest value to no further than $1.5 x$ inter-quartile range from the hinge. 
a
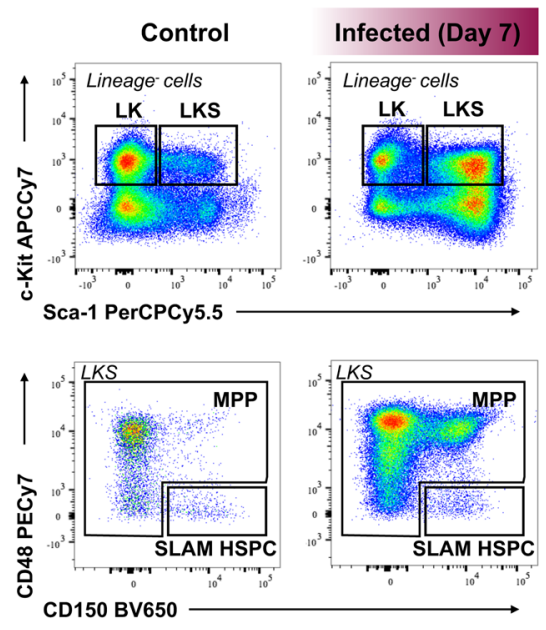

b

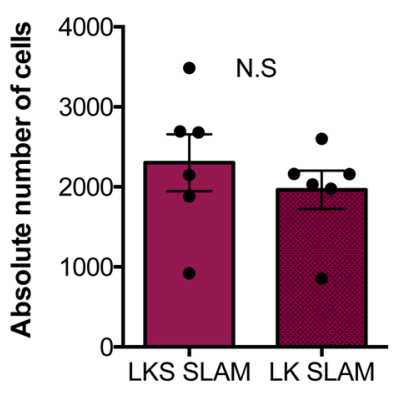

C
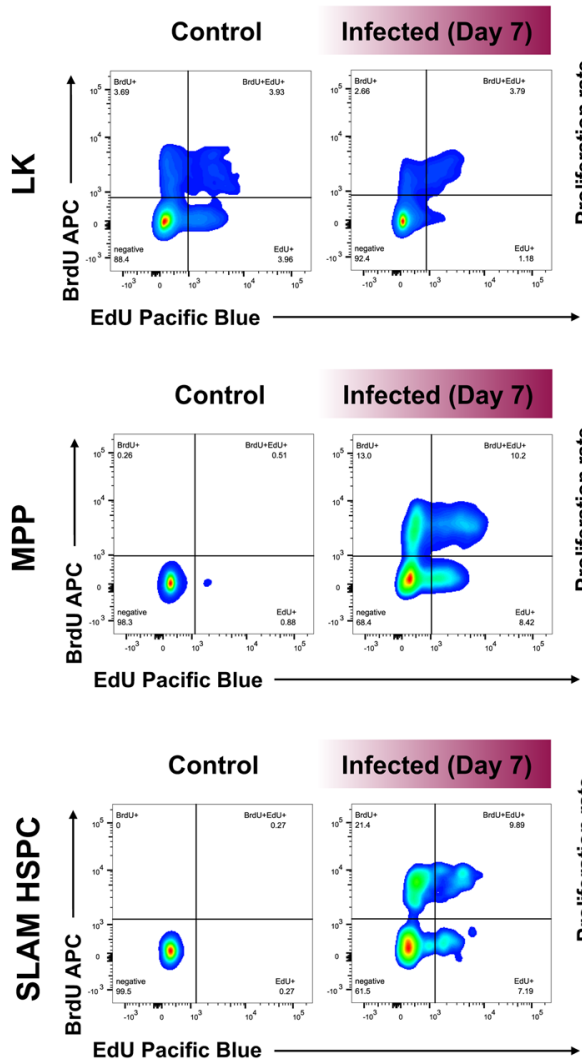

f

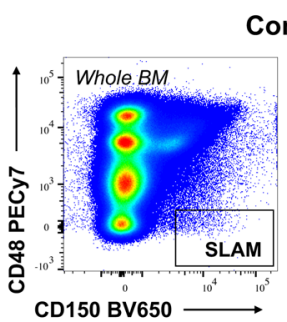

ontrol BM

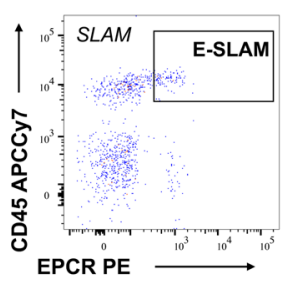

d

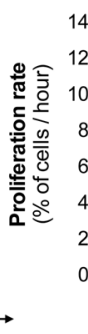

Proliferation rate
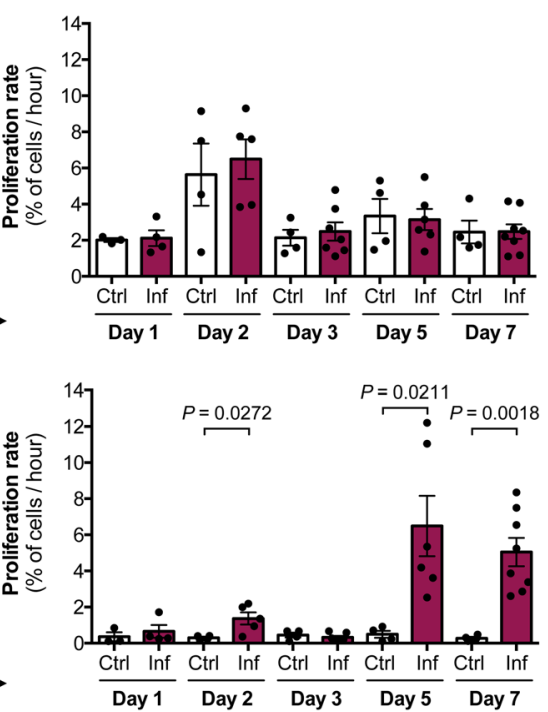

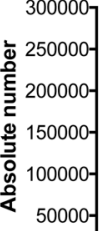

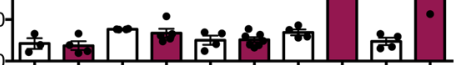

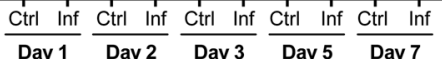
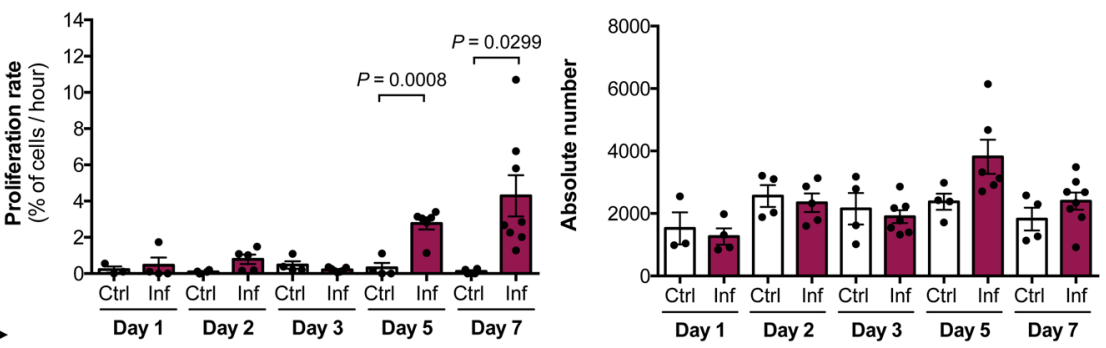

g

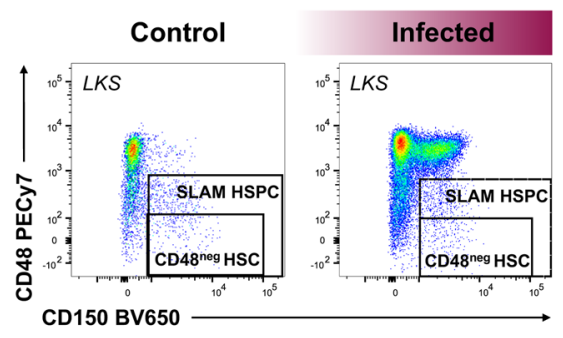

Extended Data Fig. 2 | See next page for caption. 


\section{NATURE CELL BIOLOGY}

Extended Data Fig. 2 | Quantification of instantaneous proliferation rate and size of HSPC populations. a, Representative flow cytometry plots showing the changing pattern of Sca-1 and c-Kit expression in Lineage- BM haematopoietic cells. Boxes indicate the gating strategy used for LKS, LK, MPP and SLAM HSPC compartments throughout. b, A comparison of the absolute number of SLAM HSPCs obtained when gating with (LKS SLAM) and without (LK SLAM) the marker for Sca-1 in the BM of infected mice at day 7 psi. ( $n=6$ per group). c, Representative flow cytometry plots showing the gating strategy for $\mathrm{EdU}^{+}, \mathrm{BrdU}^{+}$and $\mathrm{EdU}^{+} \mathrm{BrdU}^{+}$Lineage ${ }^{-}-\mathrm{Kit}^{+} \mathrm{Sca}-\mathrm{T}^{-}$(LK) (top panel), MPP (middle panel) and SLAM HSPC (lower panel) populations in control and infected mice at day 7 psi. d, Proliferation rates and $\mathbf{e}$, absolute numbers of LK, MPP and SLAM HSPC populations analysed by flow cytometry at day 1, 2, 3, 5 and 7 psi in control and infected mice. $n$ numbers represent individual mice and are indicated as data points on the graph. $\mathbf{f}$, Representative flow cytometry plots showing the gating strategy for E-SLAM cells (defined as CD48 neg/ow $C D 150^{+} C D 45^{+} E P C R^{++}$) in control BM. $\mathbf{g}$, Representative flow cytometry plots showing the gating strategy for $\mathrm{CD} 48^{\text {neg }} \mathrm{HSC}$ in control and infected BM. Data pooled from up to 3 independent infection experiments. All data presented as mean \pm s.e.m. $P$ values determined by unpaired two-tailed Student's $t$-tests. N.S, not significant. 
a
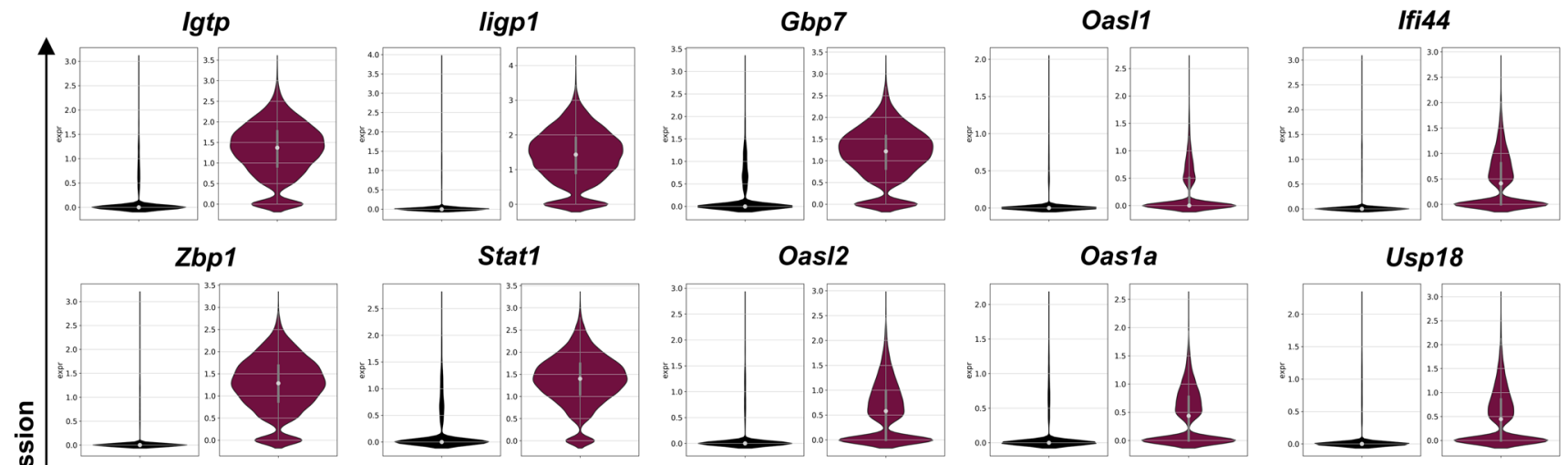

产

Cxc/10

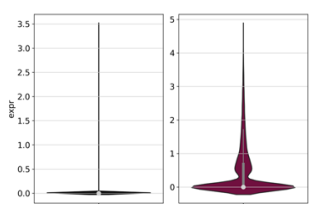

Serpina3f

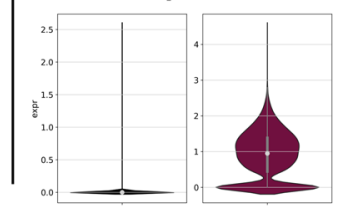

b

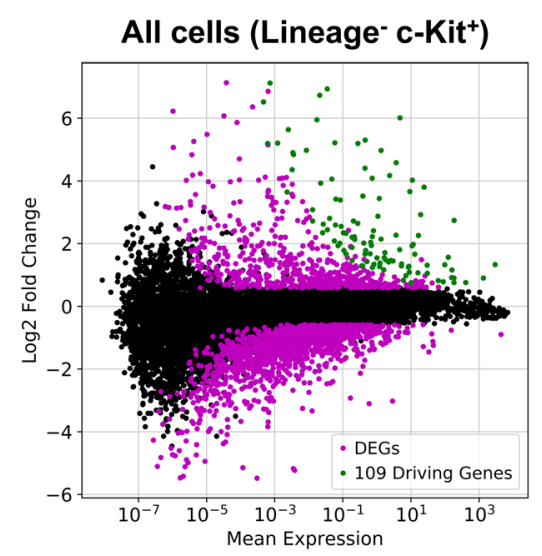

d

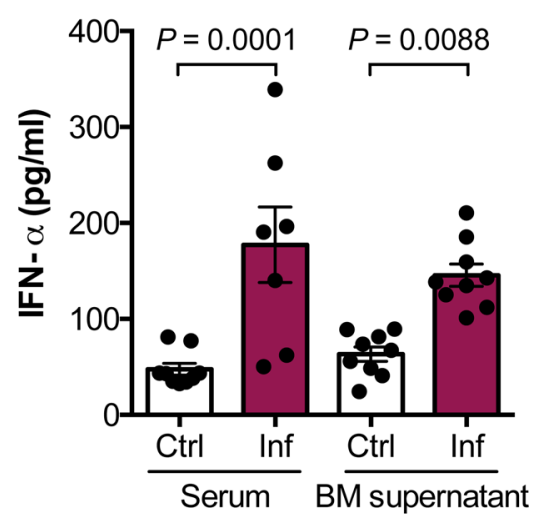

Irf7 II18bp

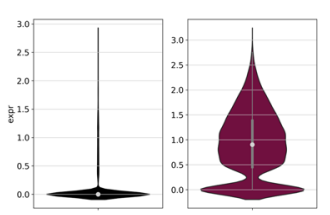

Gbp2

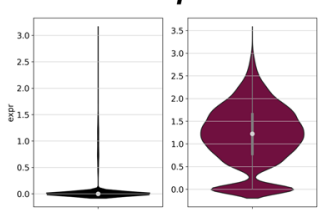

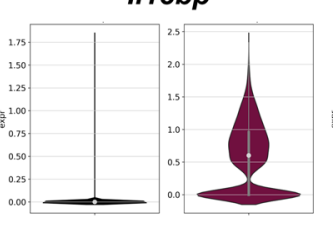

Xaf1

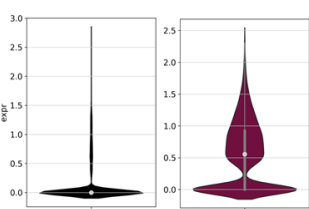

Oas3

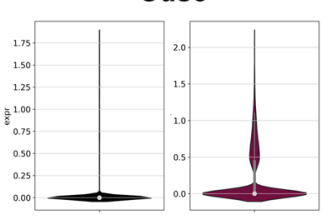

Irf1

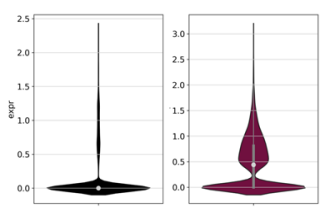

Ifi35

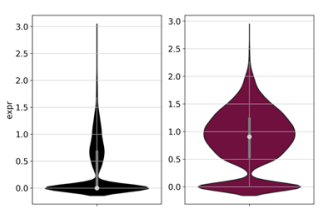

Fam26f

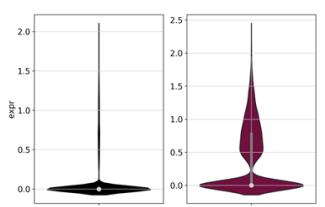

C

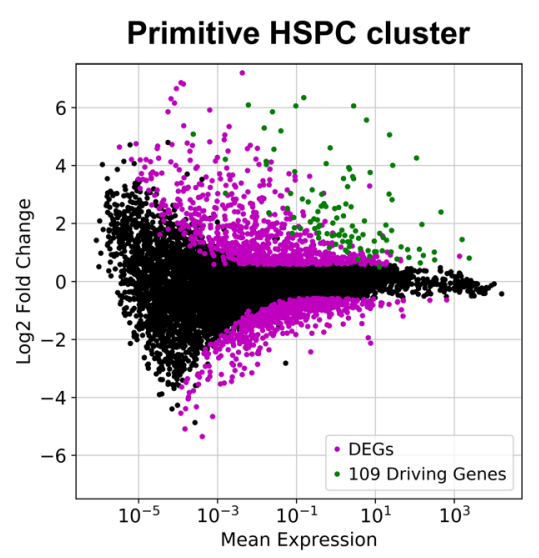

e

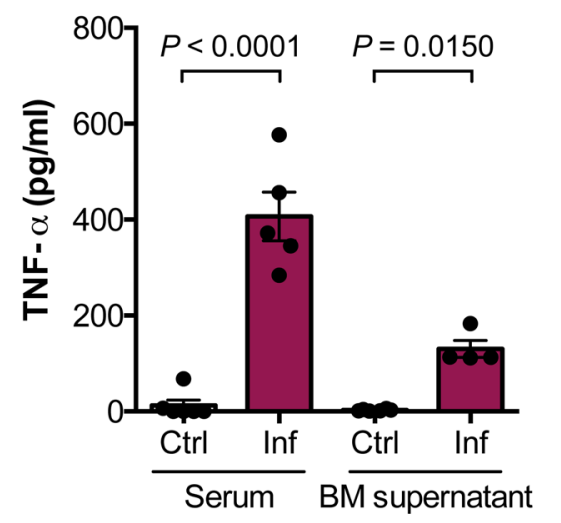

Extended Data Fig. 3 | See next page for caption. 


\section{NATURE CELL BIOLOGY}

Extended Data Fig. 3 | P. berghei triggers an inflammatory response driven primarily by IFN- $\boldsymbol{\gamma}$. a, Violin plots showing the expression of the 20 initial driving genes determined via scRNAseq analysis in control (black, left panels) and infected (maroon, right panels) mice. $n=14,193$ control cells and 13,905 infected cells. The centre dot shows the median and the box limits show the upper and lower quartiles. The whiskers extend from the box limit to the smallest and largest value to no further than $1.5 \mathrm{x}$ inter-quartile range from the hinge. MA plots demonstrating the differential expression of genes in b, all cells analysed and c, the primitive HSPC cluster specifically. All significant differentially expressed genes are highlighted in red and the 109 driving genes are highlighted in green. $\mathbf{d}$, IFN- $\alpha$ levels $(\mathrm{pg} / \mathrm{mL})$ measured by ELISA in the serum and BM supernatant of control and infected mice at day $7 \mathrm{psi}$ ( $n=9$ control, 7 infected serum and 9 infected BM supernatant samples). e, TNF- $\alpha$ levels ( $\mathrm{pg} / \mathrm{mL}$ ) measured by ELISA in the serum and BM supernatant of control and infected mice at day 7 psi ( $n=6$ control, 5 infected serum and 4 infected BM supernatant samples). Data pooled from 2 independent infection experiments ( $\mathbf{d}$ and $\mathbf{e}$ ). Data presented as mean \pm s.e.m. $P$ values determined by one-way ANOVA with post-hoc Bonferroni corrections. 
a

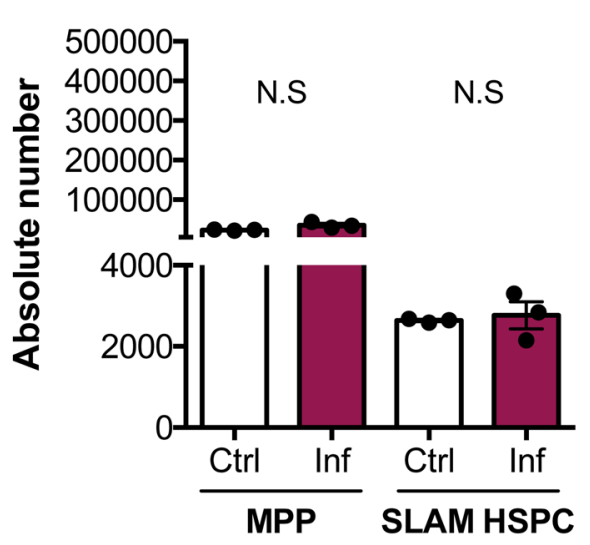

d

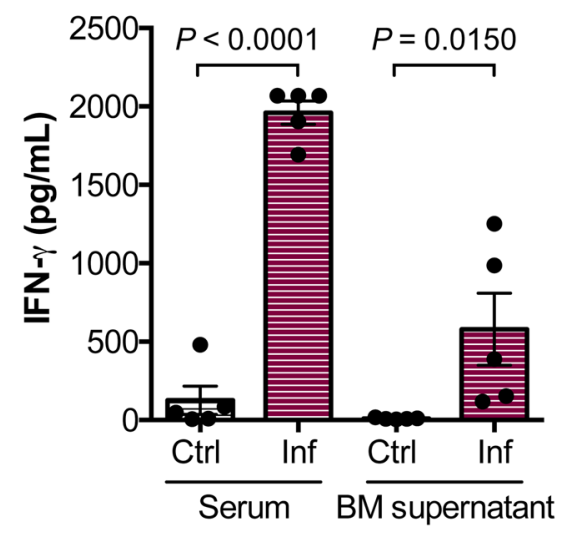

b

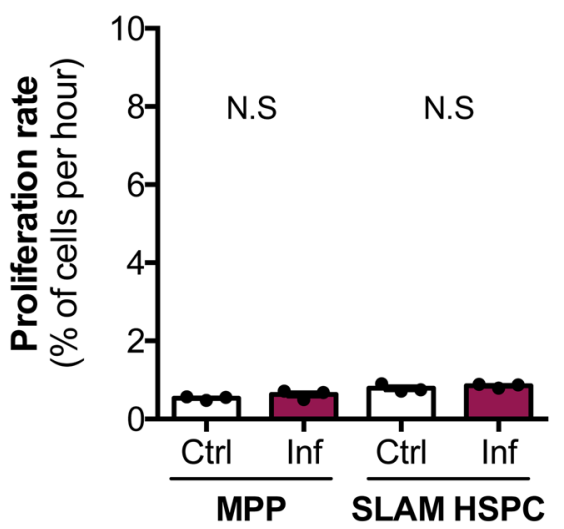

e

$\mathrm{KO}+\mathrm{WT} \rightarrow \mathrm{WT}$

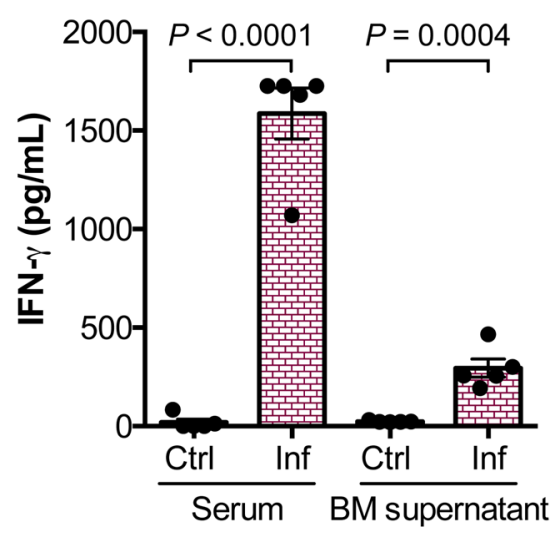

C

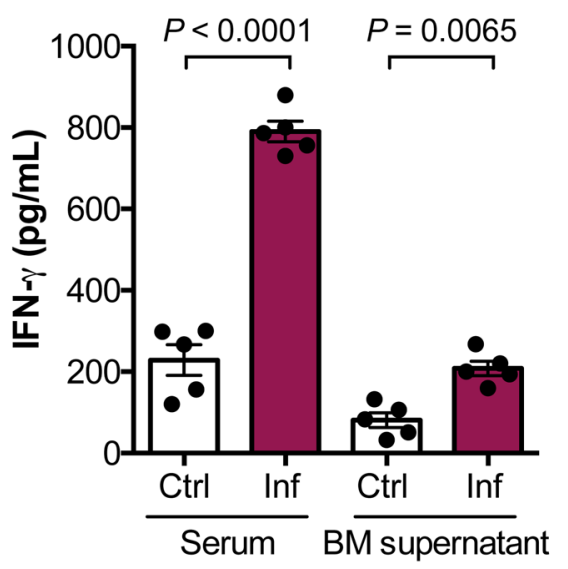

f

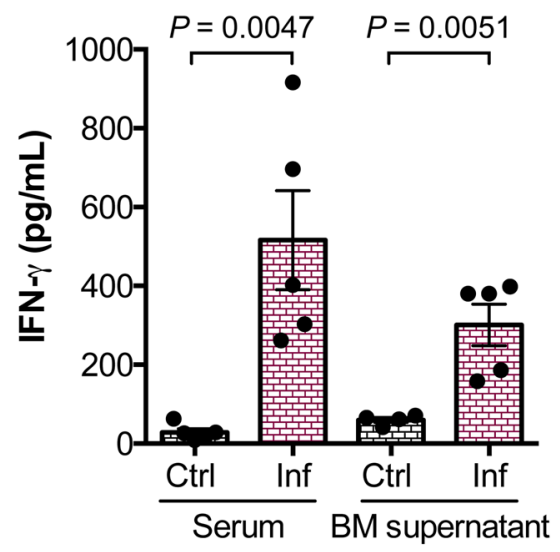

Extended Data Fig. 4 | Evaluating the effects of IFN- $\boldsymbol{\gamma}$ on HSPCs and BM stroma of $\boldsymbol{P}$. berghei-infected mice. $\mathbf{a}$, Absolute numbers and $\mathbf{b}$, proliferation rates of MPPs and SLAM HSPCs in control and infected Ifngr 1 - mice at day 7 psi ( $n=3$ per group). IFN- $\gamma$ levels (pg/mL) measured by ELISA in the serum and BM supernatant of control and infected $\mathbf{c}$, Ifngr $7^{\%}, \mathbf{d}, \mathrm{WT} \rightarrow \mathrm{KO}, \mathbf{e}, \mathrm{KO}+\mathrm{WT} \rightarrow \mathrm{WT}$ and $\mathbf{f}, \mathrm{KO}+\mathrm{WT} \rightarrow \mathrm{KO}$ chimeric mice at day $7 \mathrm{psi}(n=5$ per group). All data presented as mean \pm s.e.m. $P$ values determined by unpaired two-tailed Student's $t$-tests. N.S, not significant. 
a

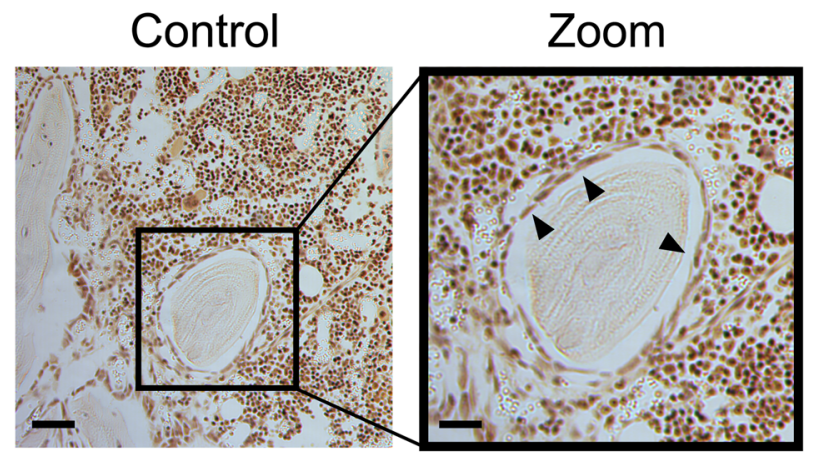

C

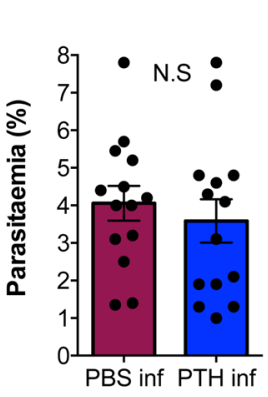

g

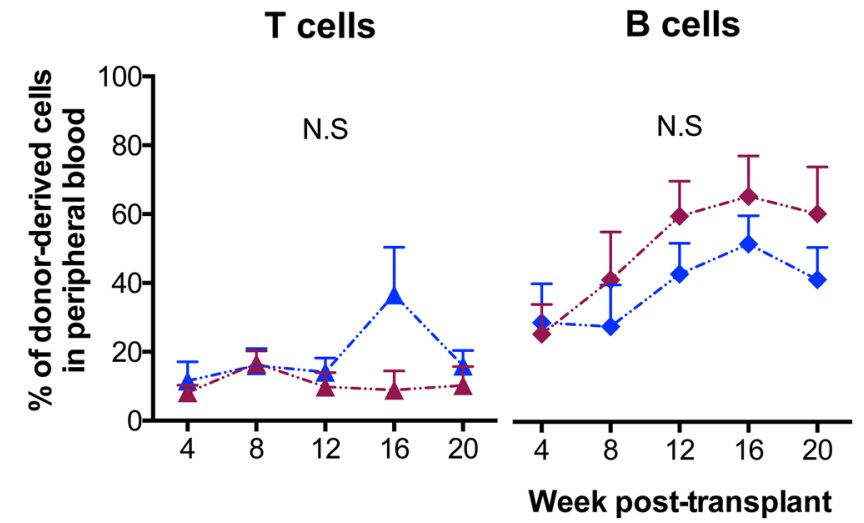

h

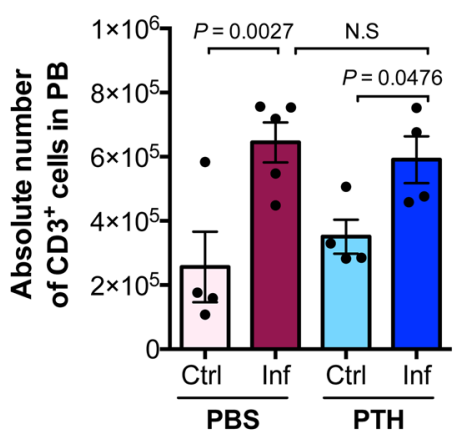

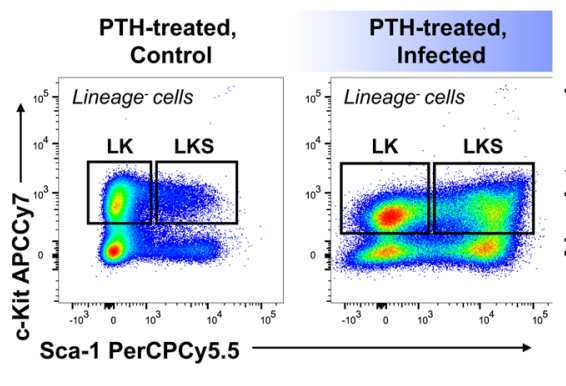

e
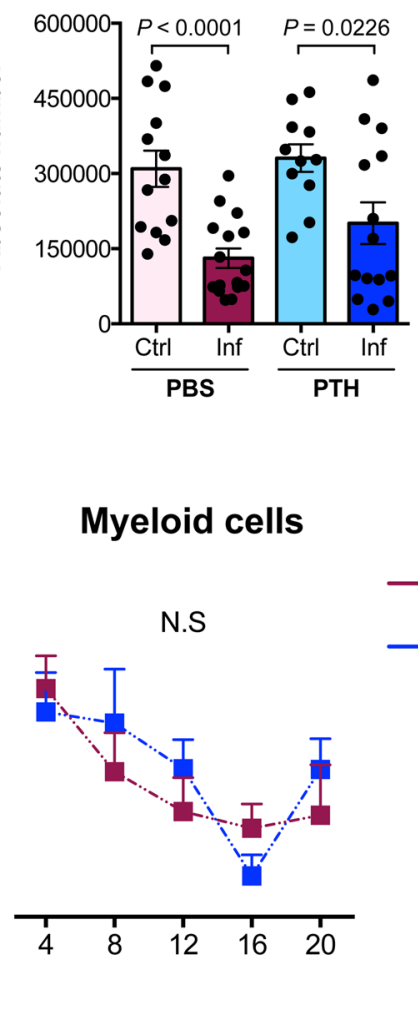

Myeloid cells

b

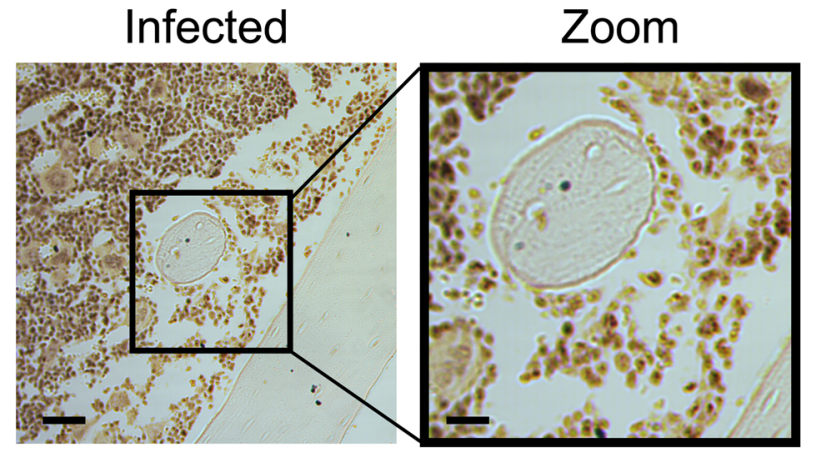

LK f

LK

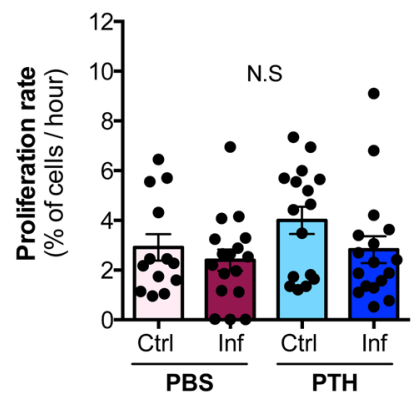

PBS-treated, Infected

PTH-treated, Infected

Extended Data Fig. 5 | See next page for caption.
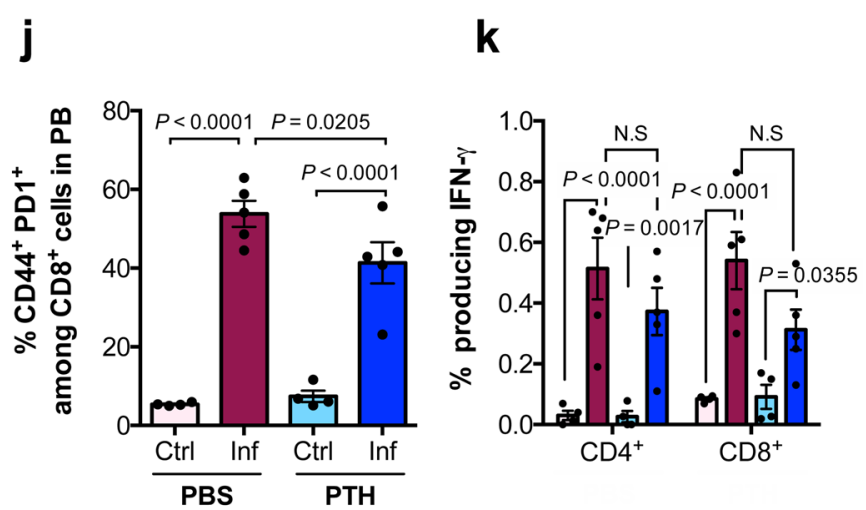
Extended Data Fig. 5 | Targeting the osteolineage with PTH during P. berghei infection. Representative images of haematoxylin and eosin stained sections of femurs from $\mathbf{a}$, control and $\mathbf{b}$, infected mice at day $7 \mathrm{psi}$. Black arrowheads indicate osteoblasts. Scale bars in control and infected images represent $50 \mu \mathrm{m}$. Scale bars in control and infected high magnification images represent $20 \mu \mathrm{m}$.c. Parasitaemia measured in peripheral blood calculated from Giemsa-stained blood films taken from PBS- $(n=14)$ and PTH-treated $(n=14)$, infected mice at day 7 psi - quantified by microscopic analysis. d, Representative flow cytometry plots of Lineage- BM haematopoietic cells in PTH-treated control and infected mice at day 7 psi. Boxes indicate the gating strategy used for LKS and LK populations throughout. e, Absolute number and $\mathbf{f}$, proliferation rate of LK cells in PBS- and PTH-treated control and infected mice at day 7 psi. $n$ numbers represent individual mice and are indicated as data points on the graph. $\mathbf{g}$, Multilineage output of transplanted SLAM HSPCs from PBS- (maroon line) and PTH-treated (blue line), infected $\mathrm{mT} / \mathrm{mG}$ donors into lethally irradiated CD45.2 recipient mice, assessed by flow cytometry up to 20 weeks after transplantation ( $n=6$ per group). $\mathbf{h}$, Absolute number of $C D 3^{+}$T-cells in peripheral blood of PBS- and PTH-treated mice. $\mathbf{i}, \mathrm{CD} 4^{+}$and $\mathrm{CD} 8^{+} \mathrm{T}$-cells as a proportion of $\mathrm{CD} 3^{+} \mathrm{T}$-cells, $\mathbf{j}$, proportion of activated $\mathrm{CD} 8^{+} \mathrm{T}$-cells and $\mathbf{k}$, proportion of $\mathrm{CD} 4^{+}$and $\mathrm{CD} 8^{+} \mathrm{T}$-cells producing IFN- $\gamma$ in peripheral blood of PBS- and PTH-treated, control and infected mice - quantified by flow cytometry. $n=4$ control, 5 infected (h-k). Data pooled from up to 6 independent infection experiments. All data shown as mean \pm s.e.m. $P$ values determined by unpaired two-tailed Student's $t$-tests (c, $\mathbf{g}$ ), one-way $(\mathbf{e}, \mathbf{f}, \mathbf{h}, \mathbf{j})$ or two-way $(\mathbf{i}, \mathbf{k})$ ANOVA with post-hoc Bonferroni corrections. N.S, not significant. 
a

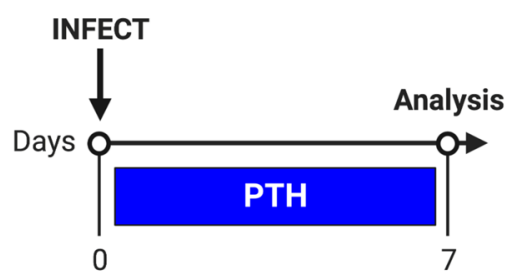

b

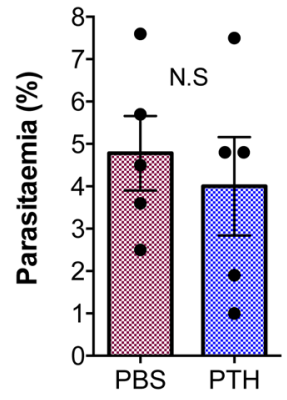

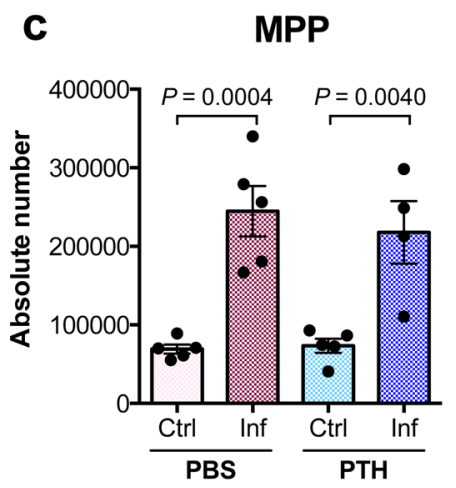

d

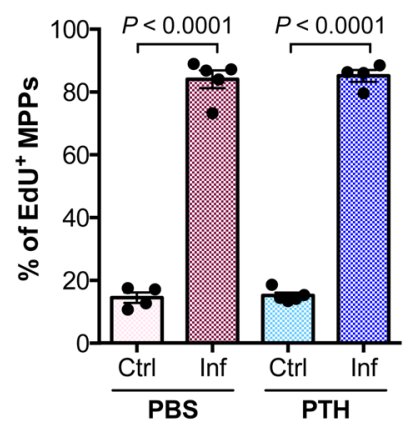

h

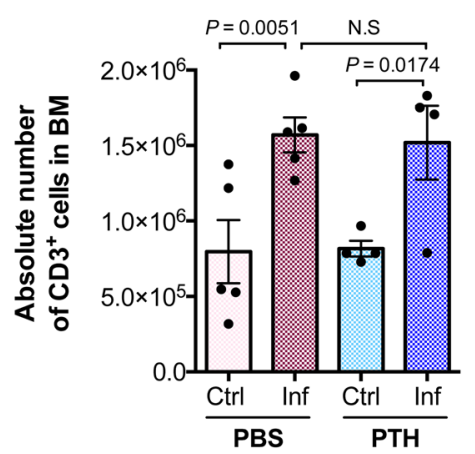

k
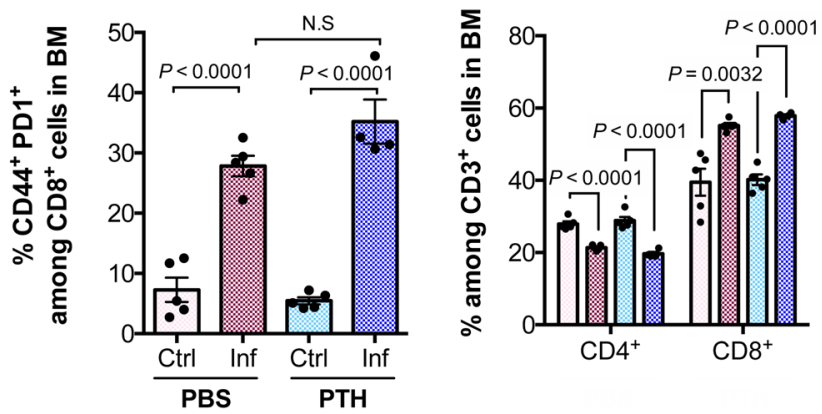

m

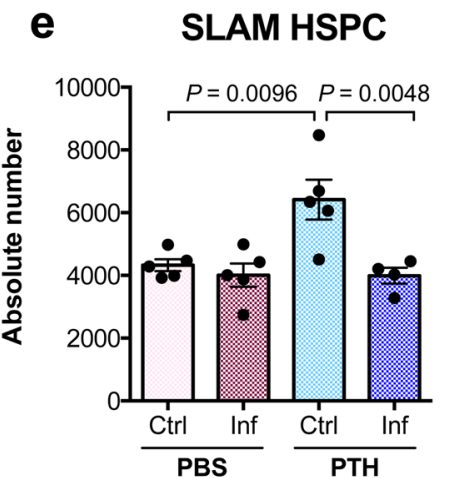

i

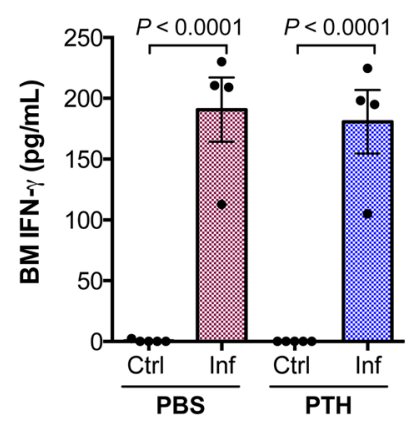

$\mathbf{n}$
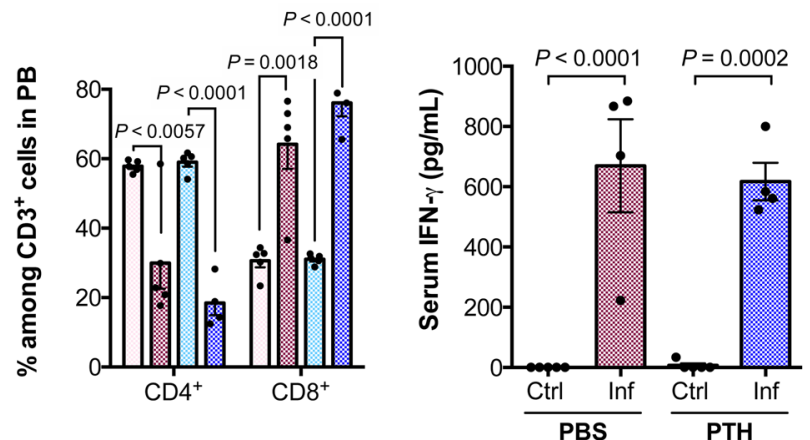

Extended Data Fig. 6 | See next page for caption. 
Extended Data Fig. 6 | Short-term PTH treatment and combined long-term PTH and NAC treatment. a, Schematic of the short-term PTH treatment regime carried out post-infection. $\mathbf{b}$, Parasitaemia measured in peripheral blood calculated from Giemsa-stained blood films taken from PBS- $(n=5)$ and PTH-treated $(n=5)$, infected mice at day $7 \mathrm{psi}$ - quantified by microscopic analysis.Absolute number of $\mathbf{c}$, MPPs and $\mathbf{e}$, SLAM HSPCs measured by flow cytometry in PBS- and PTH-treated, control and infected mice at day 7 psi ( $n>4$ per group).Proportion of EdU+ $\mathbf{d}$, MPPs and $\mathbf{f}$, SLAM HSPCs in PBS- and PTH-treated, control and infected mice at day 7 psi, measured by flow cytometry. $n$ numbers represent individual mice and are indicated as data points on the graph. Absolute number of $\mathrm{CD}^{+}{ }^{+}$-cells in the $\mathbf{g}$, BM and $\mathbf{k}$, peripheral blood of PBS- and PTH-treated mice. CD4+ and CD8 ${ }^{+} \mathrm{T}^{+}$-cells presented as a proportion of $\mathrm{CD}^{+}$T-cells in the $\mathbf{h}, \mathrm{BM}$ and $\mathbf{I}$, peripheral blood of PBS- and PTH post-infection-treated mice. Proportion of activated (defined as $\mathrm{CD} 44^{+} \mathrm{PD} 1^{+}$) CD8 ${ }^{+}$T-cells in the $\mathbf{i}, \mathrm{BM}$ and $\mathbf{m}$, peripheral blood of PBS- and PTH-treated mice. IFN- $\gamma$ levels (pg/mL) measured by ELISA in $\mathbf{j}$, BM supernatant and $\mathbf{n}$, serum from PBS- and PTH post-infection-treated mice. For $(\mathbf{g}-\mathbf{n}), n$ numbers represent individual mice and are indicated as data points on the graph. Data pooled from 2 independent infection experiments. All data presented as mean \pm s.e.m. $P$ values determined by unpaired two-tailed Student's t-tests ( $\mathbf{b}-\mathbf{f})$, one-way $(\mathbf{g}, \mathbf{h}, \mathbf{j}, \mathbf{k} \mathbf{l}, \mathbf{n})$ or two-way $(\mathbf{i}, \mathbf{m})$ ANOVA with post-hoc Bonferroni corrections. N.S, not significant. 
NATURE CELL BIOLOGY

ARTICLES

a

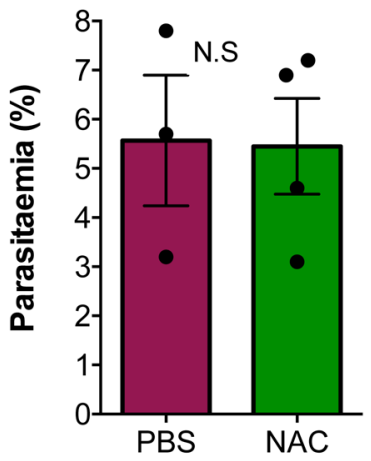

b

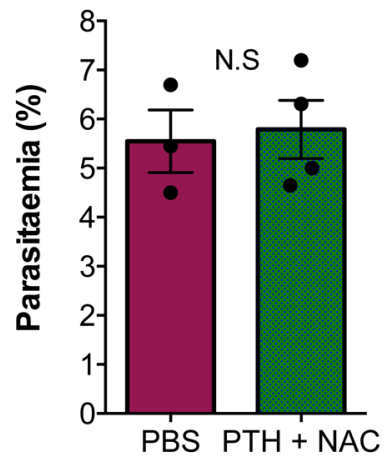

C

T cells

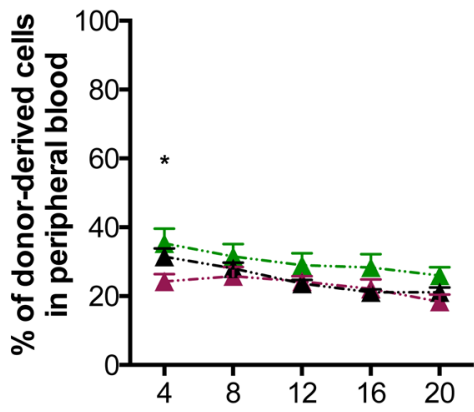

B cells Myeloid cells

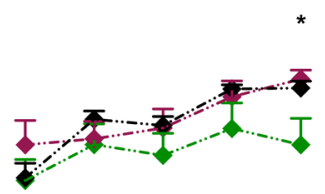

PBS-treated, Control

PBS-treated, Infected

PTH + NAC-treated, Infected

Extended Data Fig. 7 | Combined PTH+NAC treatment partially rescues HSC function. a, Parasitaemia measured in peripheral blood calculated from Giemsa-stained blood films taken from PBS- $(n=3)$ and NAC-treated $(n=4)$, infected mice at day 7 psi - quantified by microscopic analysis. b, Parasitaemia measured in peripheral blood calculated from Giemsa-stained blood films taken from PBS- $(n=3)$ and PTH+NAC-treated $(n=4)$, infected mice at day 7 psi - quantified by microscopic analysis. c, Multilineage output of transplanted SLAM HSPCs from PBS-treated, control (black line) and infected (maroon line) or PTH+NAC-treated, infected (green line) CD 45.2 donors into lethally irradiated mT/mG recipient mice, assessed by flow cytometry of tomato cells up to 20 weeks after transplantation ( $n=7$ recipients of PBS-treated control cells, 6 recipients of PBS-treated, infected cells, 7 recipients of PTH+NAC-treated, infected cells). All data presented as mean \pm s.e.m. $P$ values determined by unpaired two-tailed Student's $t$-tests $(\mathbf{a}, \mathbf{b})$ or two-way ANOVA with post-hoc Bonferroni corrections (c). N.S, not significant. In (c) PBS-treated, infected versus PTH+NAC-treated, infected T-cell comparison $P=0.0137$; PBS-treated, infected versus $P T H+N A C$-treated, infected B-cell comparison $P=0.0427$; PBS-treated, control versus PTH+NAC-treated, infected Myeloid comparison $P=0.0396$ and PBS-treated, infected versus PTH+NAC-treated, infected Myeloid comparison $P=0.0437$.

NATURE CELL BIOLOGY | www.nature.com/naturecellbiology 

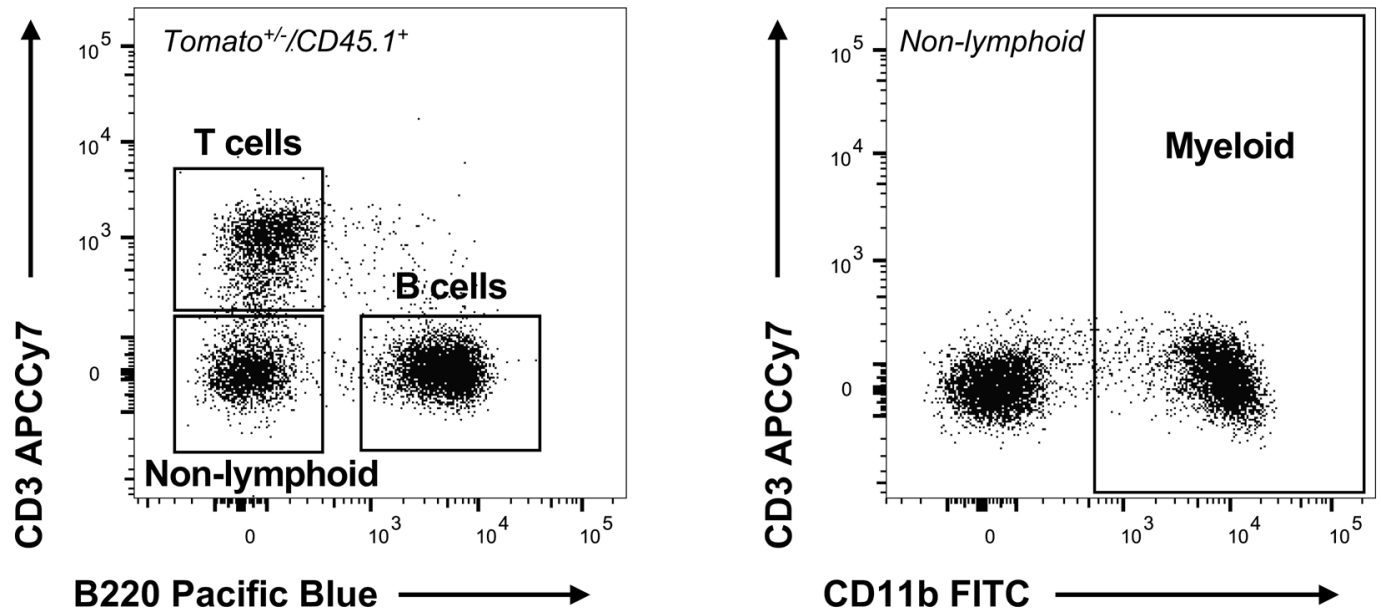

Extended Data Fig. 8 | Gating strategy for measuring lineage output in transplantation assays. Representative flow cytometry plots from the peripheral blood of transplantation assay recipient mice allowing the analysis of multilineage output after transplantation of SLAM HSPCs from control or infected donor mice (presented in Fig. 1i, Extended Data Figs. $5 \mathrm{~g}$ and 7c). Black boxes indicate the gates used after excluding debris, doublets and dead cells to gate on $T$ cells, B cells and the non-lymphoid compartment in order to isolate myeloid cells. 


\section{Control}

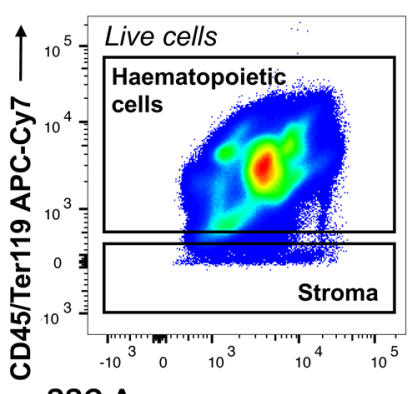

SSC-A

\section{Infected}

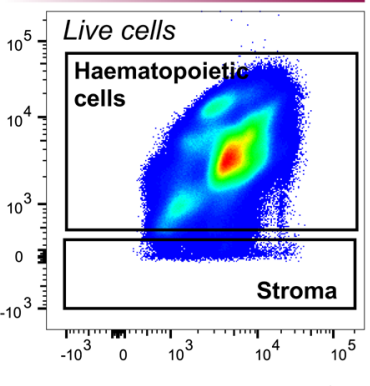

i.)

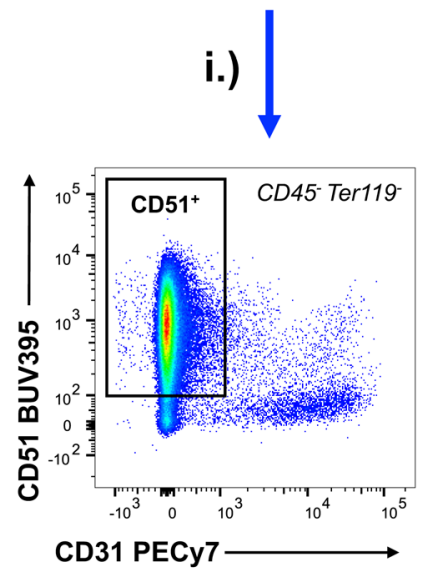

Control

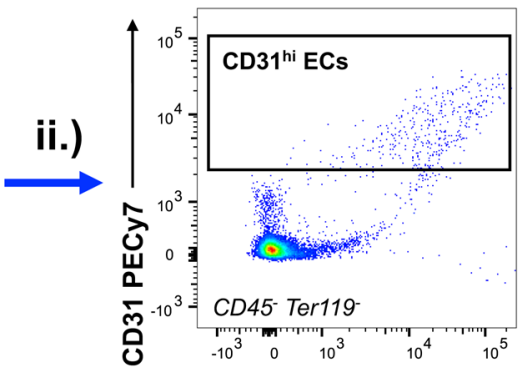

Sca-1 PcrCPCy5.5

\section{Control}

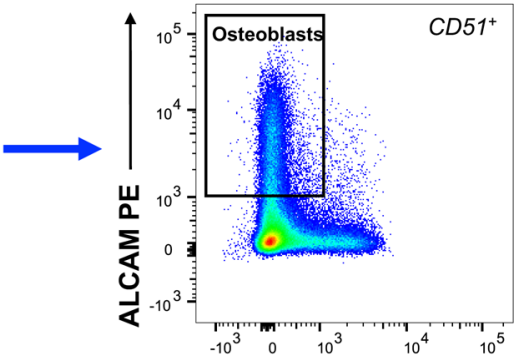

Sca-1 PerCPCy5.5
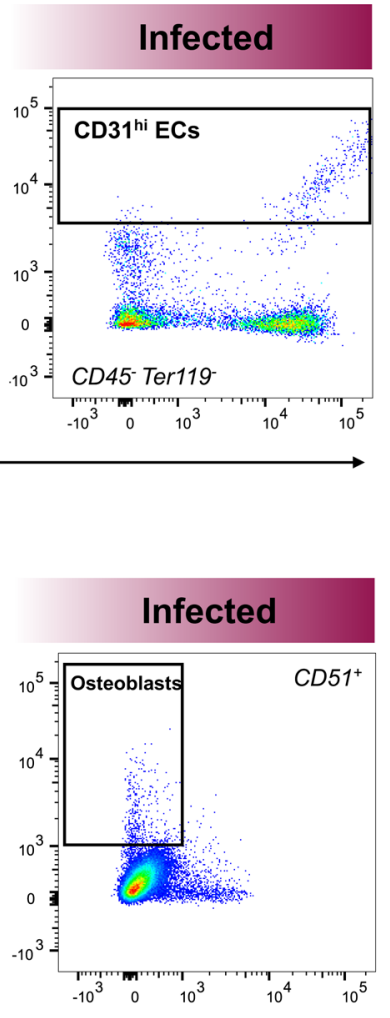

Extended Data Fig. 9 | Gating strategy for analysing stroma, endothelial cells and osteoblasts. Representative flow cytometry plots of the bone marrow of control and infected mice at day 7 psi demonstrating the gating strategy used to isolate haematopoietic cells and stroma (Fig. 3), (i) osteoblasts (Fig. 4) and (ii) endothelial cells (Fig. 5). Black boxes indicate the gates used after excluding debris, doublets and dead cells to gate on the populations labelled. 


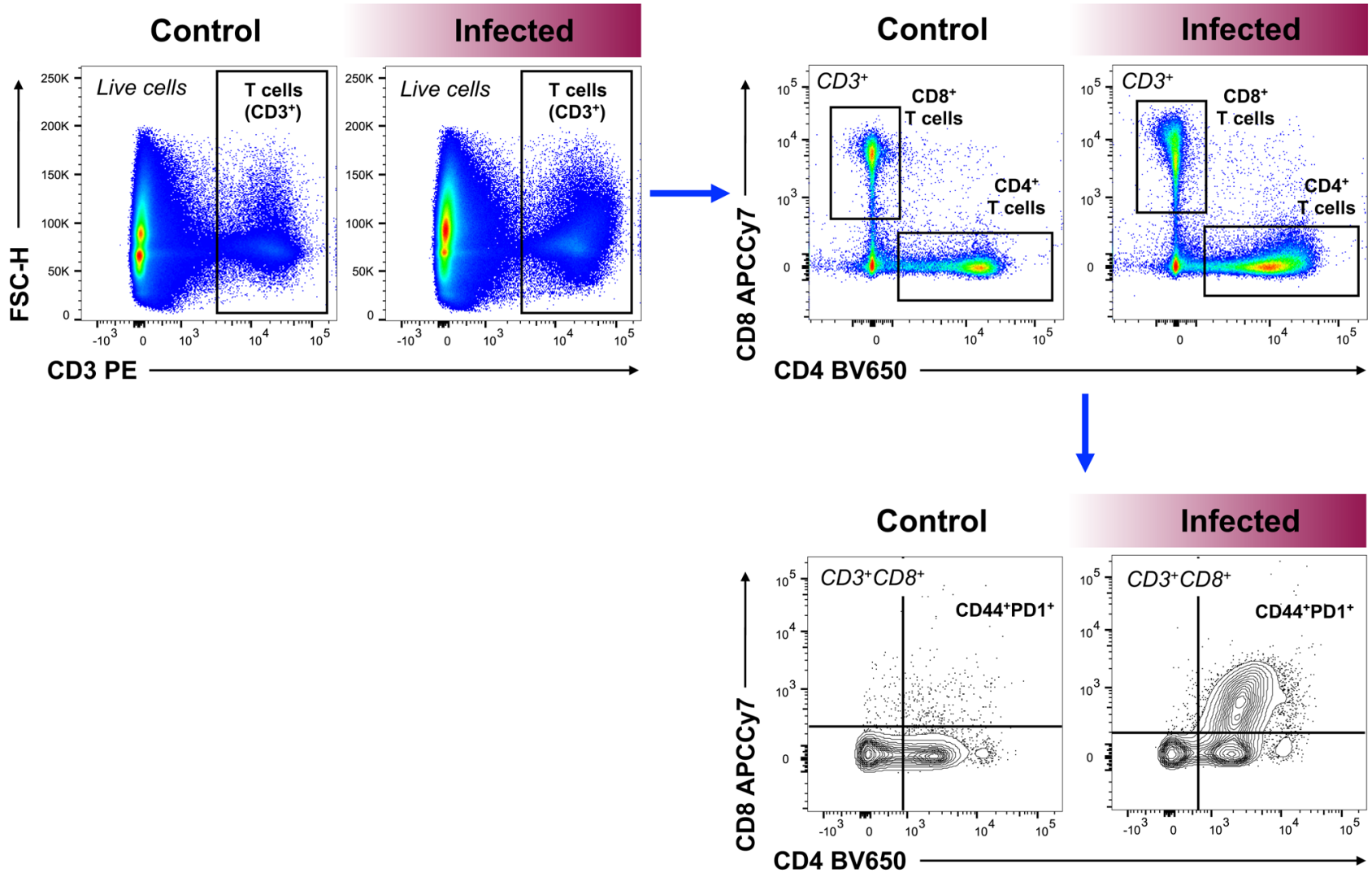

Extended Data Fig. 10 | Gating strategy for analysing T-cell subsets. Representative flow cytometry plots of the bone marrow of PBS-treated control and infected mice at day 7 psi demonstrating the gating strategy used to isolate CD3 ${ }^{+}$-cells, identify CD4 $4^{+}$and $C D 8^{+}$subsets and look at activated (defined as $\mathrm{CD}_{44}{ }^{+} \mathrm{PD1}^{+}$) CD8 ${ }^{+}$T-cells (presented in Fig. 4m-p, Extended Data Fig. $\mathrm{h}-\mathrm{k}$ and Extended Data Fig. 6g-i,k-m). Black boxes indicate the gates used after excluding debris, doublets and dead cells to gate on the populations labelled. Note: the same gating strategy was used in PTH-treated mice as well as for the peripheral blood analysis of T cells. 


\section{natureresearch}

Corresponding author(s): Cristina Lo Celso

Last updated by author(s): Sep 10, 2020

\section{Reporting Summary}

Nature Research wishes to improve the reproducibility of the work that we publish. This form provides structure for consistency and transparency in reporting. For further information on Nature Research policies, see Authors \& Referees and the Editorial Policy Checklist.

\section{Statistics}

For all statistical analyses, confirm that the following items are present in the figure legend, table legend, main text, or Methods section.

n/a Confirmed

$\bigotimes$ The exact sample size $(n)$ for each experimental group/condition, given as a discrete number and unit of measurement

$\square \bigotimes$ A statement on whether measurements were taken from distinct samples or whether the same sample was measured repeatedly

$\square$ The statistical test(s) used AND whether they are one- or two-sided

Only common tests should be described solely by name; describe more complex techniques in the Methods section

Х $\square$ A description of all covariates tested

$\square$ \ A description of any assumptions or corrections, such as tests of normality and adjustment for multiple comparisons

$\triangle$ A full description of the statistical parameters including central tendency (e.g. means) or other basic estimates (e.g. regression coefficient)

AND variation (e.g. standard deviation) or associated estimates of uncertainty (e.g. confidence intervals)

For null hypothesis testing, the test statistic (e.g. $F, t, r$ ) with confidence intervals, effect sizes, degrees of freedom and $P$ value noted

Give $P$ values as exact values whenever suitable.

Х $\square$ For Bayesian analysis, information on the choice of priors and Markov chain Monte Carlo settings

Х $\square$ For hierarchical and complex designs, identification of the appropriate level for tests and full reporting of outcomes

$\bigotimes \square$ Estimates of effect sizes (e.g. Cohen's $d$, Pearson's $r$ ), indicating how they were calculated

Our web collection on statistics for biologists contains articles on many of the points above.

\section{Software and code}

Policy information about availability of computer code

Data collection $\quad$ BD FACSDiva (v8); ZEN black image analysis software (Zeiss, v1); Libraries were sequenced with HiSeq4000 and corresponding Illumina commercial software (Illumina): NextSeq Control Software v2.2.0.4 and RTA v2.4.11 for NextSeq500 and HCS v2.2.68 for HiSeq2500

Data analysis

FlowJo (Tree Star, v10.6.1); Fiji/ImageJ (v2.0.0-rc-69/1.52n); Definiens Developer 64 (v2.0.4); GraphPad Prism (v6); Cell Ranger (v1.3); Python (v3.7); Scanpy (v1.4.5); matplotlib (v3.2.2); Seabourne (v0.10.1); R (v3.6.1); edgeR (v3.11), ggplot2 (v3.2.1); gephi (v0.9.1); EnrichR (v1); MATLAB (v9.7).

For manuscripts utilizing custom algorithms or software that are central to the research but not yet described in published literature, software must be made available to editors/reviewers. We strongly encourage code deposition in a community repository (e.g. GitHub). See the Nature Research guidelines for submitting code \& software for further information.

\section{Data}

Policy information about availability of data

All manuscripts must include a data availability statement. This statement should provide the following information, where applicable:

- Accession codes, unique identifiers, or web links for publicly available datasets

- A list of figures that have associated raw data

- A description of any restrictions on data availability

scRNAseq data that support the findings of this study have been deposited in the Gene Expression Omnibus (GEO) under accession code GSE156410. Data are also available in processed form for interactive browsing in an open access web browser. Source data underlying Figures $1-7$ and Extended Data Figures $1-7$ are provided with this study. All other data supporting the findings of this study are available from the corresponding author on reasonable request. 


\title{
Field-specific reporting
}

Please select the one below that is the best fit for your research. If you are not sure, read the appropriate sections before making your selection. $\bigotimes$ Life sciences $\quad \square$ Behavioural \& social sciences $\quad \square$ Ecological, evolutionary \& environmental sciences

For a reference copy of the document with all sections, see nature.com/documents/nr-reporting-summary-flat.pdf

\section{Life sciences study design}

All studies must disclose on these points even when the disclosure is negative.

Sample size No sample size calculations were performed. Sample sizes for each experiment were determined based on our experience, published literature and ability to obtain sufficient statistics. A minimum of 3 independent biological samples are required for statistical tests.

Data exclusions For scRNAseq, samples were filtered through pre-established quality control (QC) criteria that involved: a) fraction of total reads associated to genes; b) number of reads mapping to nuclear genes; c) percentage of mapped reads mapping to mitochondrial genes; d) percentage of mapped reads mapping to ERCCs. Only cells passing quality control filters were kept for further analysis. No data was excluded for other experiments.

Replication All findings were replicated at least once, but most often two or more times in completely independent experiments (independent batches of animals and parasites). Experiments were replicated using always same machines, instruments and softwares for data collection and analysis. Reproducibility is also addressed throughout the manuscript in the main text, methods and figure legends.

Randomization Allocation of mice to experimental groups was random, although animals were always matched in age and sex for independent experiments.

Blinding The investigators were not blinded to allocation during experiments and outcome assessment because our research group is too small to perform division of roles for blind experiments. Control samples were processed simultaneously or in parallel in all experiments.

\section{Reporting for specific materials, systems and methods}

We require information from authors about some types of materials, experimental systems and methods used in many studies. Here, indicate whether each material, system or method listed is relevant to your study. If you are not sure if a list item applies to your research, read the appropriate section before selecting a response.

Materials \& experimental systems

Methods

n/a Involved in the study

$\square \bigotimes$ Antibodies

Х Eukaryotic cell lines

\ $\square$ Palaeontology $\mathrm{n} / \mathrm{a}$ Involved in the study

\ $\square$ ChIP-seq

$\square$ Animals and other organisms

$\bigotimes \square$ Human research participants

$\bigotimes \square$ Clinical data

\section{Antibodies}

Antibodies used

\author{
From Biolegend: \\ - Biotin anti-mouse CD3e (clone: 145-2C11), cat no. 100304; dilution 1:20 \\ - APC/Cy7 anti-mouse CD3e (clone: 145-2C11), cat no. 100330; dilution 1:100 \\ - Biotin anti-mouse CD4 (clone: GK1.5), cat no. 100404; dilution 1:20 \\ - BV650 anti-mouse CD4 (clone: RM4-5), cat no. 100555; dilution 1:200 \\ - Biotin anti-mouse CD8a (clone: 53-6.7), cat no. 100704; dilution 1:20 \\ - APC/Cy7 anti-mouse CD8a (clone: 53-6.7), cat no. 100714; dilution 1:200 \\ - Biotin anti-mouse Ter119 (clone: TER119), cat no. 116204; dilution 1:20 \\ - APC/Cy7 anti-mouse Ter119 (clone: TER119), cat no. 116223; dilution 1:100 \\ - Biotin anti-mouse/human CD45R/B220 (clone: RA3-6B2), cat no. 103204; dilution 1:20 \\ - Pacific Blue anti-mouse/human CD45R/B220 (clone: RA3-6B2), cat no. 103227; dilution 1:100 \\ - PE/Cy7 anti-mouse/human CD45R/B220 (clone: RA3-6B2), cat no. 103222; dilution 1:100 \\ - Biotin anti-mouse Ly6G/Ly6C (Gr-1) (clone: RB6-8C5), cat no. 108404; dilution 1:20 \\ - Biotin anti-mouse CD11b (clone: M1/70), cat no. 101204; dilution 1:20 \\ - FITC anti-mouse CD11b (clone: M1/70), cat no. 101206; dilution 1:100 \\ - APC/Cy7 anti-mouse CD117 (c-Kit) (clone: 2B8), cat no. 105826; dilution 1:100 \\ - PerCP/Cy5.5 anti-mouse Ly6a/E (Sca-1) (clone: D7), cat no. 108124; dilution 1:100 \\ - Pacific Blue anti-mouse CD48 (clone: HM48-1), cat no. 103418; dilution 1:100
}

